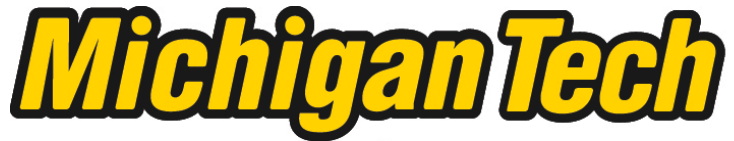 \\ Michigan Technological University Create the Future Digital Commons @ Michigan Tech
}

2015

\section{ADVANCED COMPUTATIONAL MODELING OF THE INTERNAL STRUCTURE OF SMART WIND-TURBINE BLADES}

Sanket Seetaram Bhogle

Michigan Technological University

Follow this and additional works at: https://digitalcommons.mtu.edu/etds

Part of the Engineering Commons, and the Oil, Gas, and Energy Commons Copyright 2015 Sanket Seetaram Bhogle

\section{Recommended Citation}

Bhogle, Sanket Seetaram, "ADVANCED COMPUTATIONAL MODELING OF THE INTERNAL STRUCTURE OF SMART WIND-TURBINE BLADES", Master's Thesis, Michigan Technological University, 2015.

https://doi.org/10.37099/mtu.dc.etds/907

Follow this and additional works at: https://digitalcommons.mtu.edu/etds

3 Part of the Engineering Commons, and the Oil, Gas, and Energy Commons 
ADVANCED COMPUTATIONAL MODELING OF THE INTERNAL STRUCTURE OF SMART WIND-TURBINE BLADES

\author{
By \\ Sanket Seetaram Bhogle \\ A THESIS \\ Submitted in partial fulfillment of the requirements for the degree of \\ MASTER OF SCIENCE \\ In Mechanical Engineering
}

MICHIGAN TECHNOLOGICAL UNIVERSITY

2015

(c) 2015 Sanket Seetaram Bhogle 
This thesis has been approved in partial fulfillment of the requirements for the Degree of MASTER OF SCIENCE in Mechanical Engineering.

Department of Mechanical Engineering - Engineering Mechanics

Thesis Advisor: $\quad$ Dr. Fernando Ponta

Committee Member: Dr. Lucia Gauchia

Committee Member: Dr. Leonard Bohmann

Department Chair: $\quad$ Dr. William Predebon 
To my mother, Rujuta Bhogle and father, friend and mentor, Seetaram Bhogle. 


\section{CONTENTS}

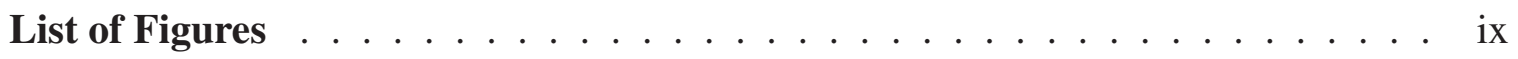

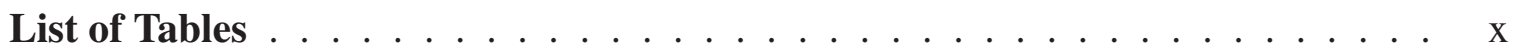

Acknowledgments ...................... xi

List of Symbols . . . . . . . . . . . . . . . . . . . . xiii

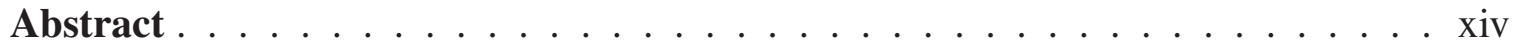

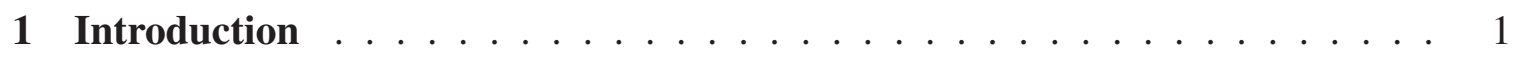

1.1 Dissertation goals ..................... 7

1.2 Dissertation Outline . . . . . . . . . . . . . . . . 8

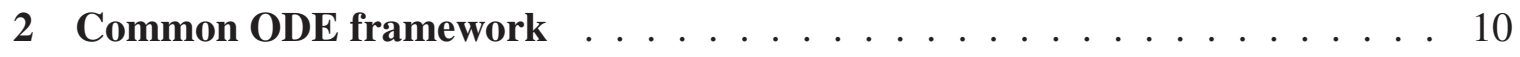

2.1 Numerical implementation . . . . . . . . . . . . . . . . 11

2.2 The Level-1 flow model . . . . . . . . . . . . . . . . . . . . . . 14

2.3 The stiff multistep BDF-ODE solver . . . . . . . . . . . . . 17

3 The Structural Model $\ldots \ldots \ldots \ldots \ldots$ 
3.1 The Generalized Timoshenko Beam Model (GTBM) . . . . . . . . . . . . 19

3.2 The $1 \mathrm{D}$ model . . . . . . . . . . . . . . . . . 23

3.3 Constitutive relations of composite materials . . . . . . . . . . . . 27

4 Internal Blade Architecture . . . . . . . . . . . . . . . . . . . . . . 34

4.1 Blade Section Structural Design . . . . . . . . . . . . . . 34

$4.1 .1 \quad$ External Spline . . . . . . . . . . . . . 35

4.1 .2 Nose Structure . . . . . . . . . . . . . 36

4.1.3 Sparcap Design . . . . . . . . . . . . . . . . 37

4.1 .4 Tail Design ........................ 37

4.1.5 Shear Web Design . . . . . . . . . . . . . . 38

5 The level-1 flow model . . . . . . . . . . . . . . . . . . . . . . . . 41

5.1 Theoretical Background: The Classic Blade Element Momentum theory . . 41

5.1.1 Corrective factors to BEM theory . . . . . . . . . . . . . . 44

5.1.2 Limitations of the classic BEM theory for large deformations . . . . 44

5.2 Large Sectional Rotation BEM (LSR-BEM) . . . . . . . . . . . . . 45

5.2.1 Iterative solution of the induction factors . . . . . . . . 51

5.2.2 Interference model for the "turbulent-wake" state . . . . . . . . . 51

5.3 The Flow Model Interface $(\mathrm{FMI}) \ldots \ldots . \ldots$. . . . . . . . . . 54

5.3.1 Pre-processing of the airfoil aerodynamic coefficients . . . . . . . . 54

5.3.2 Coupling with the structural model . . . . . . . . . . 55

5.3 .3 Wind input data ................... 55

6 Numerical Experimentation . . . . . . . . . . . . . . . . . 57 
6.1 Basic blade modelling and aerodynamic properties $\ldots \ldots \ldots 58$

6.1 .1 Blade constructive aspects $\ldots \ldots \ldots$. . . . . . . . 61

6.1 .2 Blade structural properties . . . . . . . . . . . . . . 67

6.1 .3 Turbine specifications $\ldots \ldots \ldots \ldots \ldots$. . . . . . . . . 72

6.2 Validation Tests . . . . . . . . . . . . . . . . . . . 79

$6.2 .1 \quad$ Blade Cases . . . . . . . . . . . . . . . . . . . . . . . 79

6.2 .1 .1 Original Blade . . . . . . . . . . . . . . . . 79

6.2.1.2 Superblade . . . . . . . . . . . . . . . 80

$6.2 .1 .3 \quad$ EGGshell . . . . . . . . . . . . . . . 81

6.2.1.4 HingeTwist_75_Combo . . . . . . . . . . . 83

6.2.1.5 Sweptback blades . . . . . . . . . . . . . 83

6.2.1.6 Stall blades . . . . . . . . . . . . . . . . . 84

6.2.1.7 Hinge_Twist_75_Combo_Sweptback . . . . . . . 85

6.2.1.8 Hinge_Twist_75_Combo_Stall . . . . . . . . 86

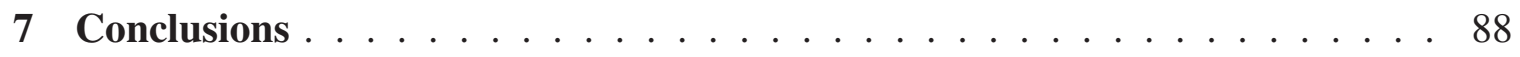

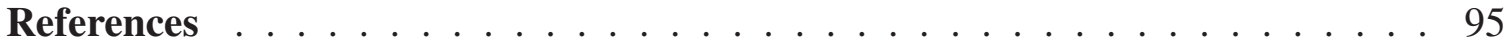

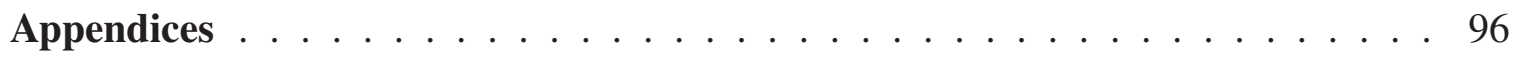

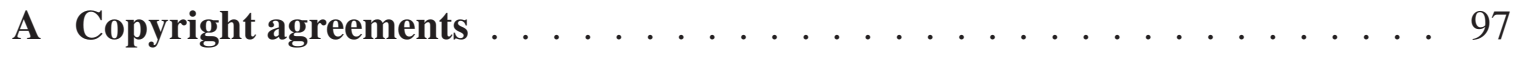

A.1 Copyright statement for Chapter $1 \ldots \ldots \ldots \ldots$

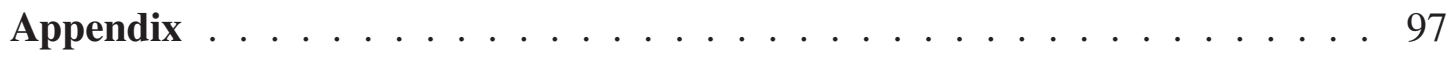




\section{LIST OF FIGURES}

1.1 Total installed wind capacity 2011-2014. An open publication by World Wind Energy Association $([1]) \ldots \ldots . \ldots 2$

1.2 Contribution of different countries to the total world production . . . . . . . 2

1.3 Newly constructed windmills D4 (nearest) to D1 on the Thornton Bank, 28 $\mathrm{km}$ off shore, on the Belgian part of the North Sea. The windmills are 157 $\mathrm{m}$ (+TAW) high, $184 \mathrm{~m}$ above the sea bottom. . . . . . . . . . . 3

1.4 REPower $5 \mathrm{M}$ wind turbine blades. . . . . . . . . . . . . . . 5

1.5 Wind-turbine blades convoy on the way to Scout Moor wind farm, England. 6

2.1 Pseudo-code diagram. . . . . . . . . . . . . . . . 11

2.2 Common ODE framework. . . . . . . . . . . . . . . . 13

2.3 The Pseudo-code diagram corresponding to the common framework on the Level-1 flow model. . . . . . . . . . . . . . . . . . 16

3.1 Example of blade-section structural architecture representative of current commercial blade designs. . . . . . . . . . . . . . . . . 18

3.2 Explanation of the Generalized Timoshenko theory with a schematic showing the orthogonal triads,reference line, and beam pre and post-deformation. 20

3.3 The reference frames for a laminate and their orientations. . . . . . . . 33

4.1 A concept of D-Spar internal configuration . . . . . . . . . . 35 
4.2 A concept of I-Beam internal configuration $\ldots \ldots \ldots \ldots$

4.3 First step in creation of the airfoil External Airfoil Creation . . . . . . 36

4.4 Internal structure of the airfoil showing the split in the Nose structure (Upper Half). . . . . . . . . . . . . . . . . . . . . 36

4.5 Internal structure of the airfoil showing the split in the Nose structure. . . . 37

4.6 Internal structure of the airfoil showing the sparcap structure. . . . . . . . 37

4.7 Internal structure of the airfoil showing the blunt tail section with the split . 38

4.8 Internal structure of the airfoil showing the pointed tail section with the split 38

4.9 Internal structure of the airfoil showing shear web structure $\ldots \ldots \ldots 38$

4.10 Pointed tail airfoil with new internal structure $\ldots \ldots \ldots$. . . . . 40

4.11 Blunt tail airfoil with new internal structure $\ldots \ldots \ldots$. . . . . . . 40

5.1 Aerodynamic efforts over the airfoil. . . . . . . . . . . . . . . . 42

5.2 HAWT blade cross-sectional analysis. . . . . . . . . . . . . . 42

5.3 Hub coordinate system. . . . . . . . . . . . . . . . . 46

5.4 Tilt and cone angles definition. . . . . . . . . . . . . . 47

5.5 Blade coordinate system. . . . . . . . . . . . . . . . . . 48

5.6 Fitting the Lock experimental data. . . . . . . . . . . . . . . . . 52

5.7 Power law relation fitting Lock experimental data. . . . . . . . . . . . 53

6.1 Distribution of chord along the blade. . . . . . . . . . . . 58

6.2 Distribution of chord along the stall blade. . . . . . . . . . . 58

6.3 Profiles of ellipsoidal sections $\ldots \ldots \ldots \ldots \ldots \ldots$

6.4 Profiles of airfoil master sections $\ldots \ldots \ldots$. . . . . . . . . . . 62

6.5 Aerodynamic coefficients for standard airfoils . . . . . . . . . . . . . 63 
6.6 Morphed sections' profiles . . . . . . . . . . . . . . 67

6.7 Finite element meshes . . . . . . . . . . . . . . . . . . . 74

6.8 Finite element meshes for master sections (a). . . . . . . . . . . . 75

6.9 Finite element meshes for the master sections (b) . . . . . . . . . . . 76

6.10 Finite element meshes for the master sections (c) . . . . . . . . . . . 77

6.11 Top view of the internal blade layout. . . . . . . . . . . . . . 78

6.12 Power output of Original and Superblade. . . . . . . . . . . . . . . . 81

6.13 Power output of Original and EGGshell. . . . . . . . . . . . . . . 82

6.14 Power output of Original and HingeTwist_Combo_ 75. . . . . . . . . . 83

6.15 Power output of Original,Sweptback, and Stall blades. . . . . . . . . . . . 84

6.16 Power output of Sweptback and Hinge_Twist_75_Combo_Sweptback. . 86

6.17 Power output of Stall, ComboStall and Hinge_Twist_75_Combo_Stall. . 87 


\section{LIST OF TABLES}

1.1 Repower 5MW wind turbine specifications. . . . . . . . . . . . . 4

5.1 Mean Square Error (MSE) with the different empirical approximations to Lock's experimental data. . . . . . . . . . . . . . . . . . 53

6.1 Distributed blade aerodynamic properties. . . . . . . . . . . . . . . 59

6.2 Distributed blade aerodynamic properties-Stall blade. . . . . . . . . . . . 60

6.3 Structural details. . . . . . . . . . . . . . . . . . 65

6.4 Baseline Blade Material Properties . . . . . . . . . . . . . . 66

6.5 Structural properties for the Blade section . . . . . . . . . . . . . 69

6.6 Distributed blade structural properties . . . . . . . . . . . 71

6.7 Global turbine parameters . . . . . . . . . . . . . . 73 


\section{ACKNOWLEDGMENTS}

I want to thank my advisor, Dr. Fernando Ponta for his guidance and support during my research years. I will always cherish the long discussions in the research lab. All these days in the research lab not only did develop me as student of science but also made me a grow as a human being. I would also treasure those sailing sessions, besides being great fun they were also great learning sessions.

A big thank you to my family for being there for me through my thick and thin.

I would also like to take this as an opportunity to thank my wind energy research group for being some of the finest people to work along. I would like to thank Anurag Rajan to help us understand the language of wind turbines when it was all alien for us. I am also grateful to Nimish Deshpande and Muraleekrishnan Menon for helping me out in my thesis and research. I would also like to thank Dr. Alejandro Otero for his invaluable inputs towards my research.

Finally I thank Michigan Technological University for being such a great school to study at. 


\section{List OF SYMBOLS}

\section{Symbols in Chapter 5}

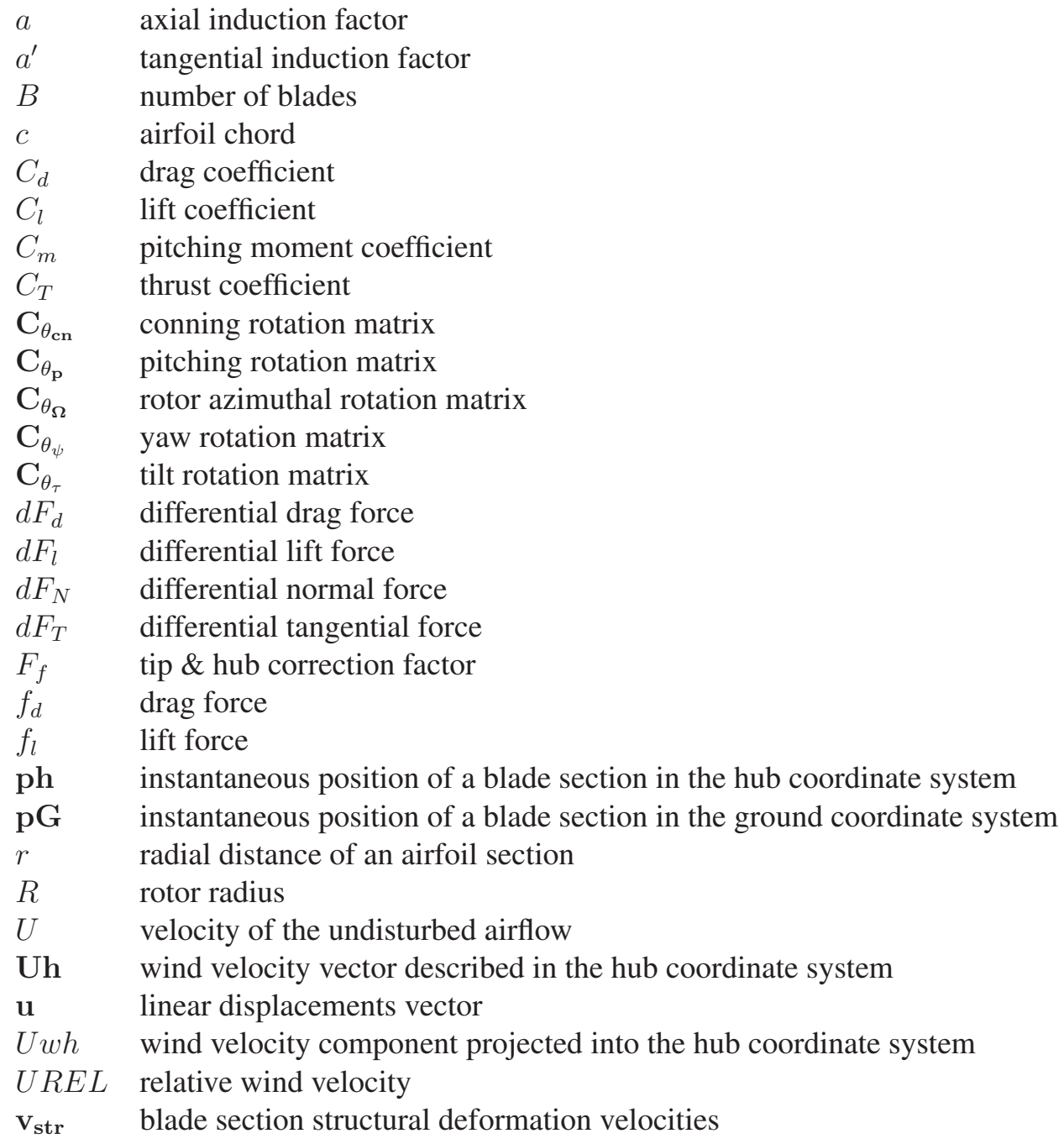




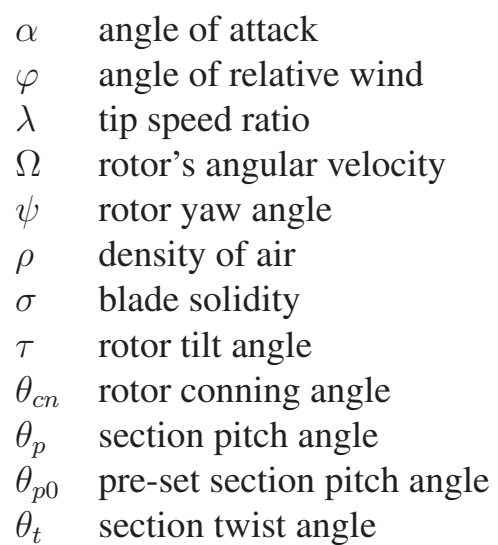

\section{Symbols in Chapter 3}

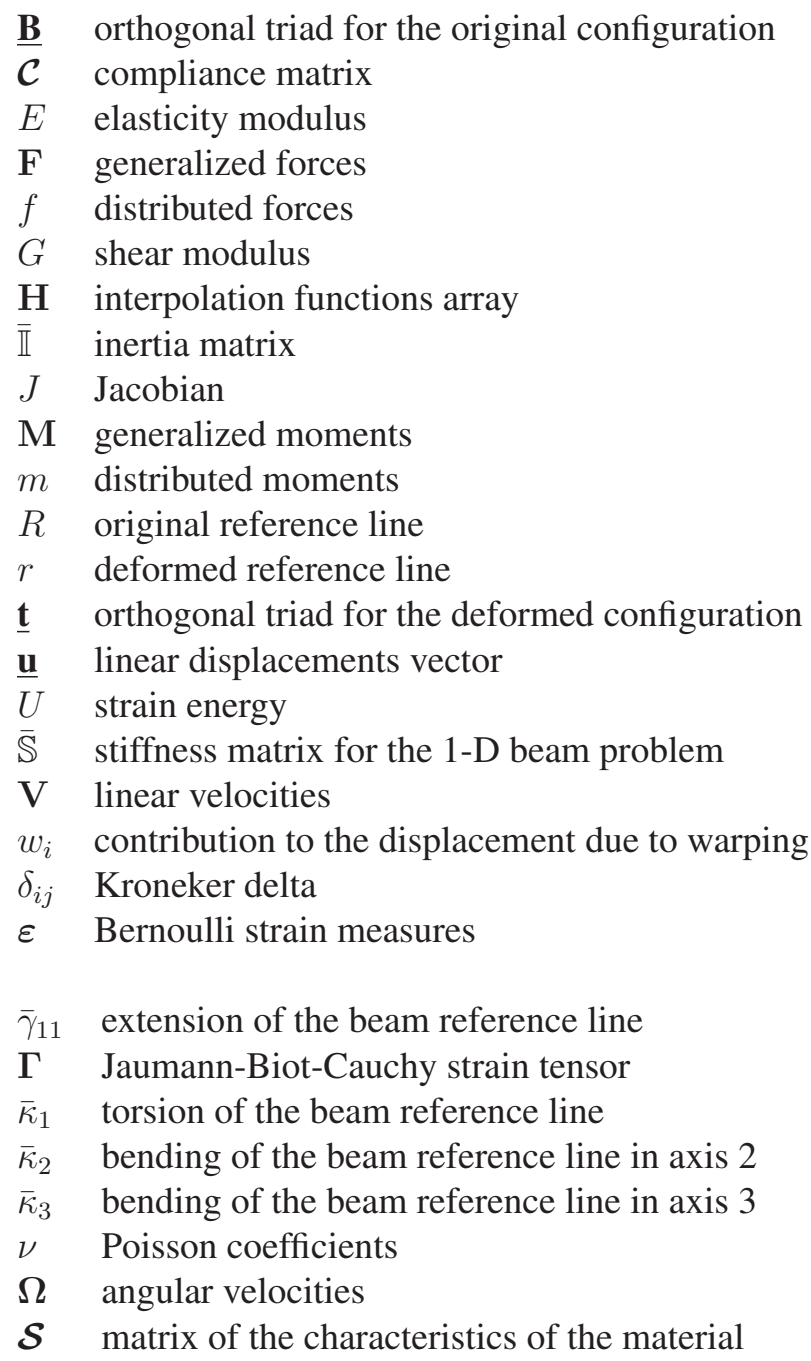




\title{
Advanced Computational Modeling of the Internal Structure of Smart Wind-Turbine Blades
}

\author{
Sanket Seetaram Bhogle \\ Michigan Technological University, 2015 \\ Advisor: Dr. Fernando Ponta
}

\begin{abstract}
Implementation of stable aeroelastic models with the ability to capture the complex features of Multi concept smartblades is a prime step in reducing the uncertainties that come along with blade dynamics. The numerical simulations of fluid structure interaction can thus be used to test a realistic scenarios comprising of full-scale blades at a reasonably low computational cost

A code which was a combination of two advanced numerical models was designed and was run with the help of paralell HPC supercomputer platform. The first model was based on a variation of dimensional reduction technique proposed by Hodges and Yu. This model was the one to record the structural response of heterogenous composite blades. This technique reduces the geometrical complexities of the heterogenous blade section into a stiffness matrix for an equivalent beam. This derived equivalent 1-D strain energy matrix is similar to the actual 3-D strain energy matrix in an asymptotic sense. As this 1-D matrix helps in accurately modeling the blade structure as a 1-D finite element problem, this substantially redues the computational effort and subsequently the computational cost that are required to model the structural dynamics at each step. Second model comprises of implementation of the Blade Element Momentum Theory. In this approach we map all the velocities and the forces with the help of orthogonal matrices that help in capturing the large deformations and the effects of rotations in calculating the aerodynamic forces. This ultimately helps us to take into account the complex flexo torsional deformations.

In this thesis we have succesfully tested these computayinal tools developed by MTU's research team lead by for the aero elastic analysis of wind-turbine blades. The validation in this thesis is majorly based on several experiments done on NREL-5MW blade, as this is widely accepted as a benchmark blade in the wind industry. Along with the use of this innovative model the internal blade structure was also changed to ad up to the existing benefits of the already advanced numerical models.
\end{abstract}




\section{INTRODUCTION}

Wind energy has its roots centuries ago disguised as windmills. It was the industrial revolution that led o the rise of thermal power. This new found thermal power generation was a more reliable source of electricity thus making wind energy obsolete for we the people. The traditional windmill applications of water pumping corn grinding etc were also replaced by more efficient machines driven by electricity. This made the wind energy to assume a back seat for several decades. A Danish pioneer Poul la Cour during the late 19th century showed that wind energy can also be used in production of electricity however the low costs of procuring coal and oil worked against the favor of wind power. The increasing energy demands and the environmental effects caused by the conventional energy production methods led mankind to wander back into unconventional ways of energy production. This led to the rebirth of wind energy. Wind energy these days is one of the important supplier of energy to the grid connected electricity globally. Wind power is considered to be one of the most rapidly growing energy production methods all thanks to the considerable progress in research and technology revolving around wind power. The worldwide wind capacity has reached 336,327 $M W$ by the end of June 2014 out of which 17,613 MW were added in the first half of the year itself.This increase in the wind capacity is observed to be more than the growth in first half of the years 2013 and 2012.1.1 Around 4\% of the world's energy demands will be fulfilled by wind power itself by mid-2014. (WWEA) [2]. The top wind markets for 2014 were China, Germany, Brazil, India and USA.(See Fig 1.2) 


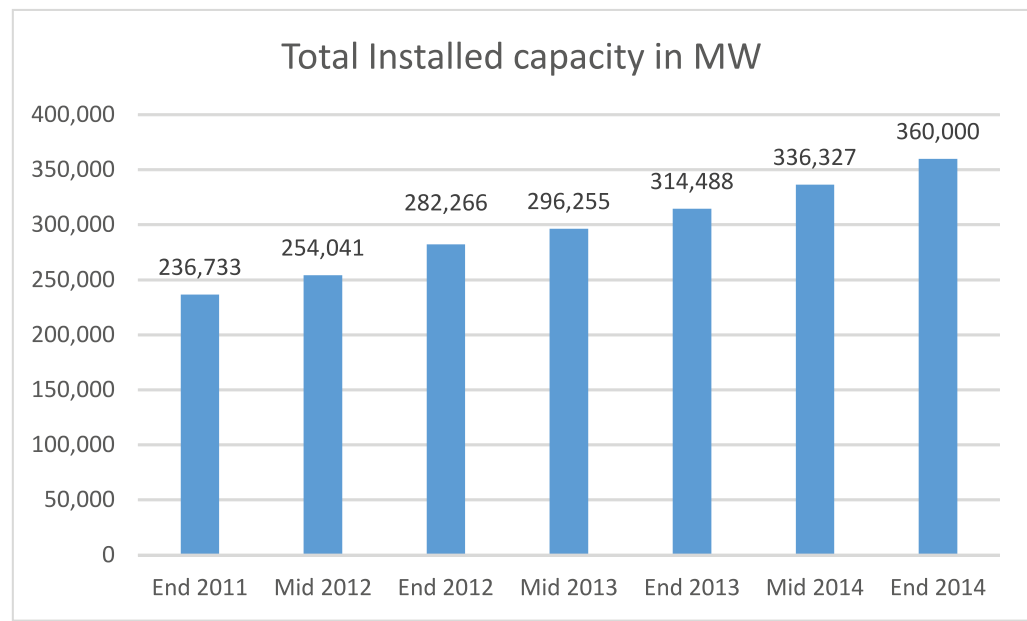

Figure 1.1. Total installed wind capacity 2011-2014 as per World Wind Energy Association Association([1]).

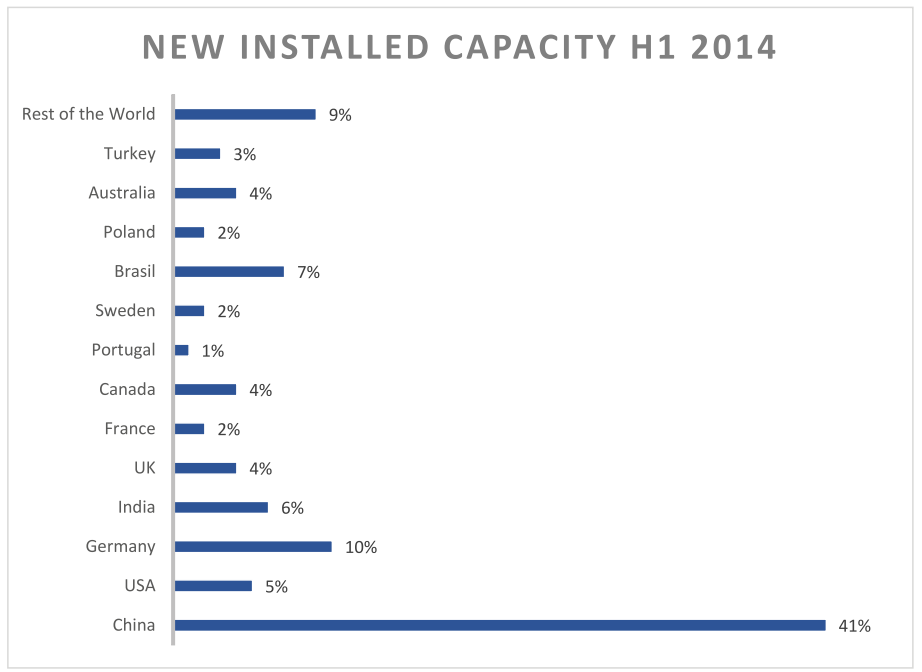

Figure 1.2. Contribution of different countries to the total world production as per World Wind Energy Association.([1])

The last three decades have shown, the only way in increasing the capacity of energy production is by increasing the size of the machine. The development of wind industry propagated a wave of development in the technology sector [3]. The data and the information obtained from each power class of turbines was used in development of the next and bigger machine. This process is termed as upscaling. Economics of scale factor reflects a substantial drop in wind energy production due to the rise in the size of the machine. 


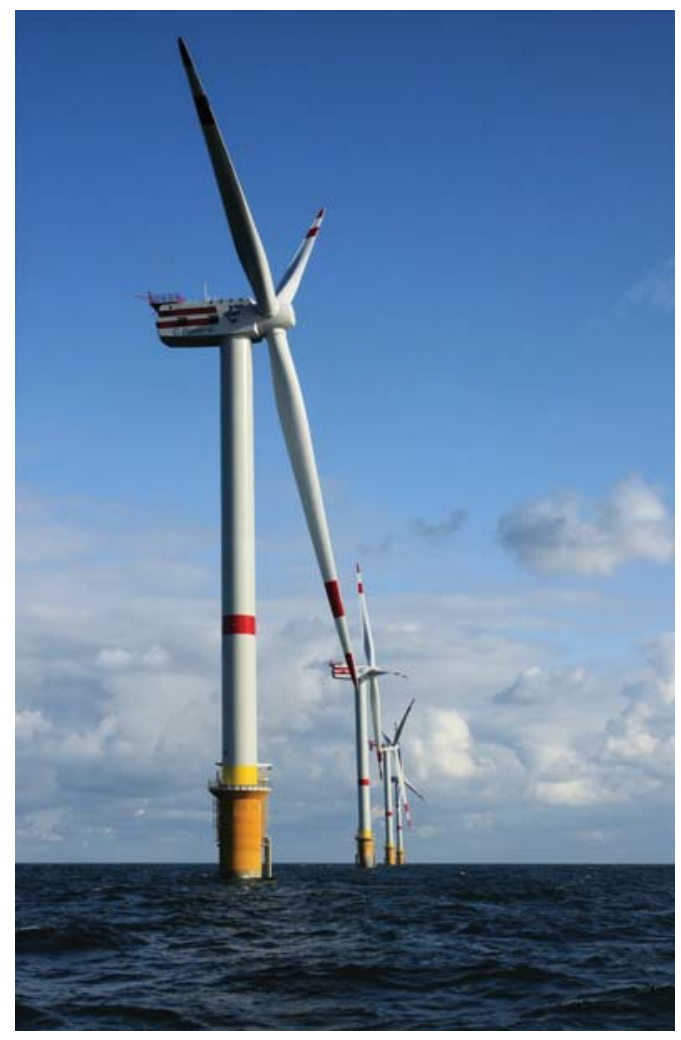

(a)

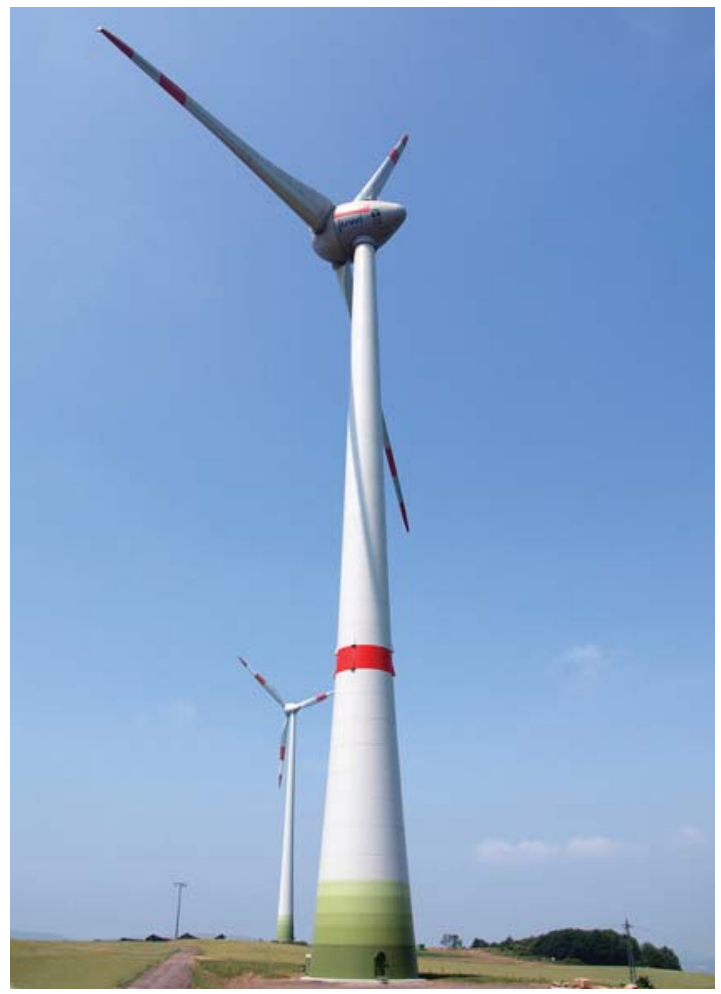

(b)

Figure 1.3. Newly constructed windmills D4 (nearest) to D1 on the Thornton Bank, $28 \mathrm{~km}$ off shore, on the Belgian part of the North Sea. The windmills are $157 \mathrm{~m}$ (+TAW) high, $184 \mathrm{~m}$ above the sea bottom.: (a) REpower 5M, with an output power of $5 \mathrm{MW}$ and a rotor diameter of 126 $m$. Picture taken by Hans Hillewaert. (b) The wind energy park S̈chneebergerhof in Germany (Rhineland-Palatinate). In the center a wind turbine Enercon E-126 (7.5 MW) by Kuebi = Armin KÃijbelbeck. Copyright statement in Appendix A.

Such state of the art machines are manufactured mainly by companies like GE, Vestas, RE-Power, Enercon, and Siemens. The power output of the machines produced by these manufacturers lie anywhere between 3.6 $\mathrm{MW}$ to $7.5 \mathrm{MW}$. Figures $1.3 \mathrm{a}$ and $1.3 \mathrm{~b}$ show Repower 5M and Enercon E-126 respectively. Repower 5M generates $5 \mathrm{MW}$ of power with a rotor diameter of $126 \mathrm{~m}$ for both offshore as well as onshore installations. Enercon E-126 [4] can generate a power output of 7.5 MW with a rotor diameter of $127 \mathrm{~m}$ for all inland installations.

There is a word that the wind turbine industry is now aiming at development of next generation of wind turbines. These machines will be huge off shore installations with a power output of about $12 \mathrm{MW}$ and would have a rotor diameter of $200 \mathrm{~m}$. If the made out successfully these super turbines will substantially reduce the cost of wind energy production. 
Table 1.1

Repower 5MW wind turbine specifications.

\begin{tabular}{ll}
\hline Design & \\
Rated Power & $5000 \mathrm{~kW}$ \\
Cut-In Wind Speed & $3.5 \mathrm{~m} / \mathrm{s}$ \\
Rated Wind speed & $13 \mathrm{~m} / \mathrm{s}$ \\
Cut-Out Wind Speed & \\
$\quad$ Offshore Version & $30 \mathrm{~m} / \mathrm{s}$ \\
$\quad$ Onshore Version & $25 \mathrm{~m} / \mathrm{s}$ \\
Rotor & \\
Diameter & $126 \mathrm{~m}$ \\
Speed Range & $6.9-12.1 \mathrm{rpm}$ \\
Blades & \\
Length & $61.5 \mathrm{~m}$ \\
\hline
\end{tabular}


In addition to this development Chen et al [5] suggested that in sites that turn out to be favorable, competitive production of hydrogen as a substitute fuel can as be carried out.

As discussed earlier the upscaling of wind turbines led to cheaper generation of the wind power, the upscaling of the blades presented a challenge of their own. Wind power industry today provides its consumers with an array of innovative concepts. The wind turbines have surely come a long way from fixed speed stall controlled devices to variable speed pitch control turbines. However upscaling these blades has exhibited problems of its own from a design stand point as well as a manufacturing, transportation and installation stand point. Current blade manufacturing process involves a composite laminate based approach and requires a skilled work force along with being labor intensive. These limitations in blade manufacturing has generated a bottleneck in the industry. It is due to this bottleneck that the wind energy sector hasn't bloomed to its full potential. Upscaling also remains less efficient as, such a complex production process has led to an increase in the manufacturing cost of the rotor.

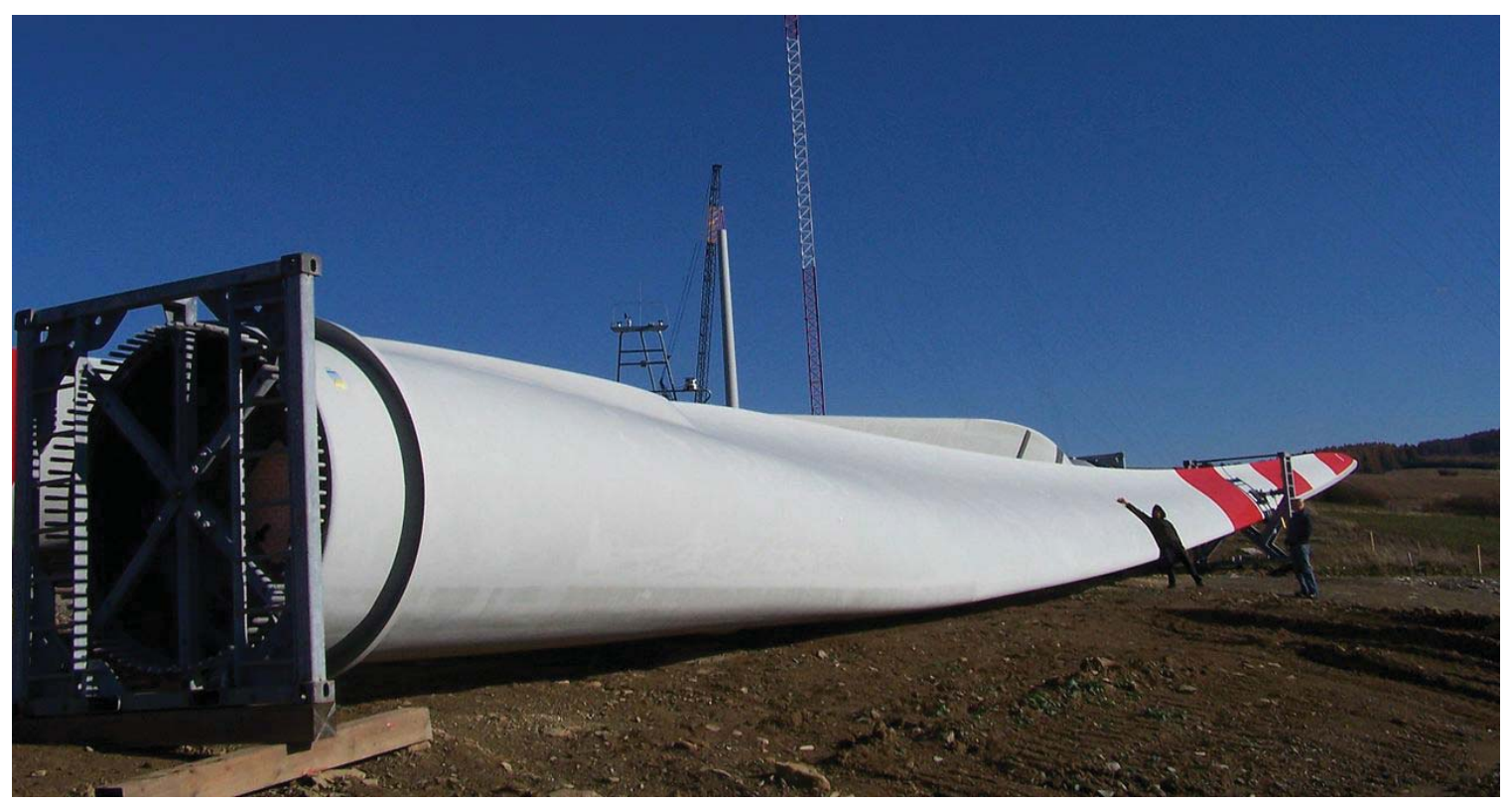

Figure 1.4. REPower 5M wind turbine blades. Wind farm installation at Bukowsko-Nowotaniec, Poland. Picture taken by Korona B. Copyright statement in Appendix A.

A completely new array of problems is exhibited when one looks at this problem from a transportation as well as a logistic stand point. Upscaling of these blades obviously increases the size of these wind turbine blades. Such increase in size of the wind turbine blades as shoots up the installation as well as the transportation costs for the same. The argument that wind turbines are produced in parts and then assembled at the installation 
site is also void in this scenario as a blade is one huge monolithic chunk of material. From a design stand point researchers aren't still successful in creating a blade in parts that exhibits the properties of a monolithic blade. Limitations of the crane capacity is also another problem from the installation point. These practical problem thus put a direct limitation to the upscaling process there by hindering the real power production potential. Practical limitations thus severely compromise the designing of wind turbine blades.

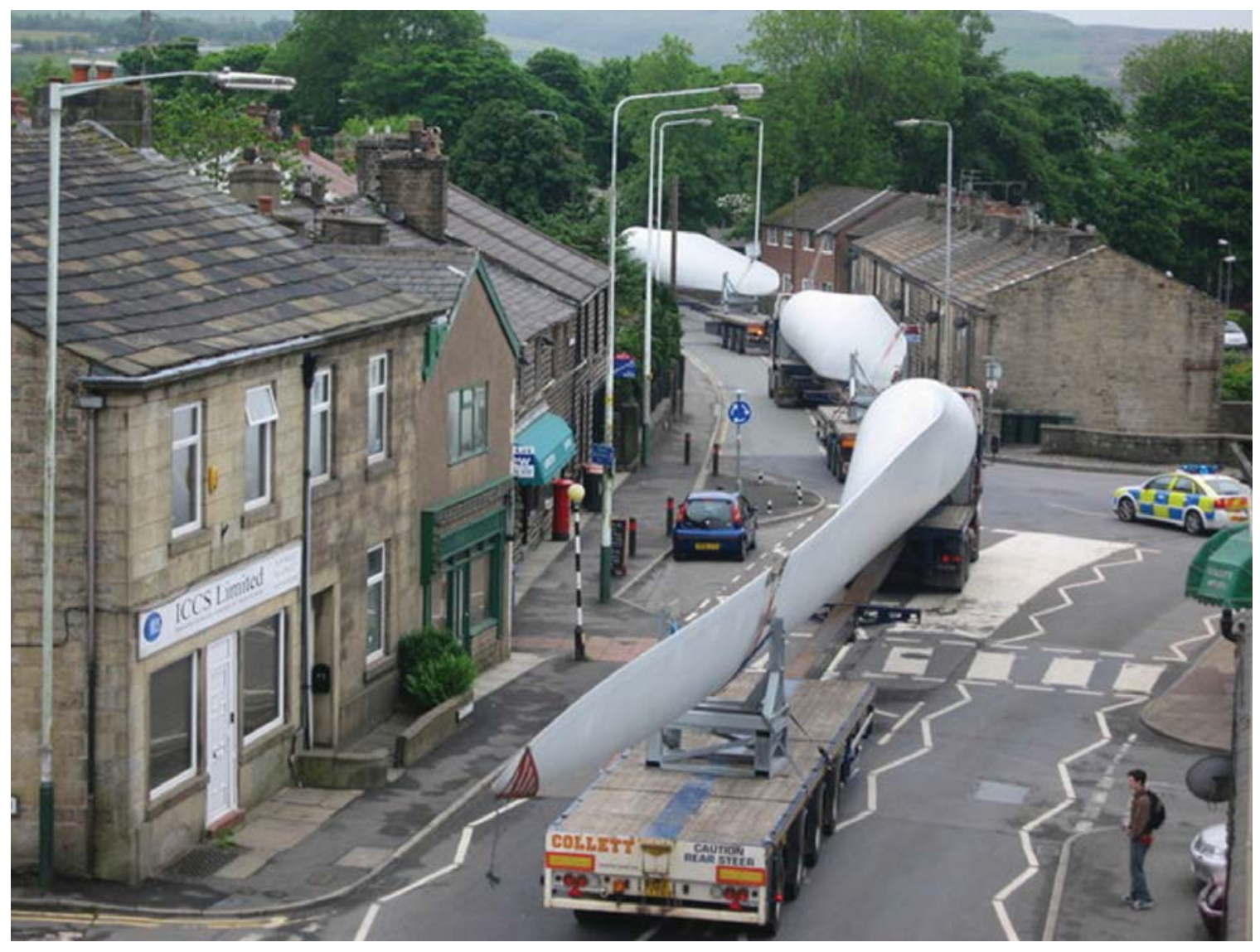

Figure 1.5. Wind-turbine blades convoy on the way to Scout Moor wind farm, England. Picture taken by Paul Anderson. Copyright statement in Appendix A.

The blades during their period of operation are acted upon by a combination of fluctuating loads. These combined with the considerable size variations makes it difficult for us to obtain reliable extrapolated data from the wind turbine experiments. The vortex creations and shading of it in their wake is also a result of oscillations and deformation of the blades. This decides the fluctuations in the aerodynamic forces that are acted upon the wing thereby creating a cycle of events involving fluid structure interactions. It is thus of utmost importance that one must have a keen understanding of the possible fluid structure 
interactions that before designing the blades as well as all other aerodynamic surfaces that are to be modeled. The computational models of FSI are thus used for design as well as optimization of the blade design. Due to this complexities it is very evident that the wind industry has been hesitant in introducing new concepts and new design practices in the design of aerodynamic surfaces as they do not ensure reliability as of yet. Innovations in the near future are likely to introduce material adpativeness, structural response which may end up requiring a different control strategies.

\subsection{Dissertation goals}

For simultaneous analysis of the presented aeroelastic problem coupled with flexotorsional effects and material adaptiveness it is necessary to develop a control strategy involving of computationally effective self-adapting algorithms which could be run in parallel on HPC supercomputer platform. A structural response model involving the use of Variational Asymptotic Beam Sectional techniques is used to reduce the complex geometry exhibited by the blade section and its material properties into a single stiffness matrix of a 1-D beam. These equations obtained for the 1-D beam problem are then solved using a non -adaptive Ordinary differential Equation (ODE) solver. More on this solver is further explained in Chapters 2 and 5.

The structural model is eventually combined with three different types of flow models. These models differ from each other on the level of description provided in its solution and also the usage of computational resources. This dissertation work involves an in-depth use of Level-1 flow model and expands upon the work of previous work by MTU's reserach team lead by Dr. Fernando Ponta [6] [7] [8]. The dissertation mainly deals with analysis of materially adaptive wind turbine blades by changing the internal structure of the airfoil along with playing upon the material properties of the blades by shuffling the material composition of blades.

The use of a nonlinear adaptive ODE algorithm provides a common framework to control the time-dependant solution for the aeroelastic problem. Equations modeling the dynamics of the control system and electro-mechanical devices may also be added to the general ODE system, with the control actions and the electrical network dynamics modifying the boundary conditions for the aeroelastic solution. This provides a natural way of integrating the structural response and the dynamics of the control system into the general solution of the flow problem in a process constantly controlled by the same self-adaptive ODE algorithm.

Non-linear adaptive ODE solver is also used so that the common ODE framework provides us with a time dependent solution of the aeroelastic problem under consideration. 


\subsection{Dissertation Outline}

This dissertation is divided into five parts. Chapter 2 reflects the set of algorithms that have been used for the computational purposes. The chapter also helps us understand the working of the Common ODE framework and the implementation of the same to control the interactions of various computational models in modelling the complex physical behavior of the blade sections. This whole computational process is governed and controlled by one self-adapting ODE algorithm which monitors the local truncation error at every timestep and thus helps in improving the efficiency and also ensuring the stability of the time marching problem it is solving. All the modules of this framework were written in MATLAB language, mainly because of the universality it offered and the amount of flexibility of coding. Using MATLAB for all coding purposes also ensured inter-connectivity between the different modules of the framework. This modular approach treated each module independently thereby promoting a parallel approach to the solution rather than sequential solving of the problem. Due to thus independent modular approach adding to as well as modifying the existing modules has become easier. Bottom line, this modular approach has helped us in creating a parallel solution to the aeroelastic time marching problem using various control strategies that is all bundled up together into one computationally efficient self-adaptive algorithm.

Chapter 5 sheds more light on Level-1 flow model. This flow model helps in providing the aerodynamic loads on the blade section. This Level-1 flow model is sensitive enough to record the complex deformation modes that the structural model is able to provide. The feedback which we obtain from the Level-1 flow model must now be of the same description as the one provided by the structural model to reliably mimic the fluid structure interactions on the rotor blades. The Level-1 flow model is built up on the BEM theory and incorporates the linear operators along with performing the rotation of the physical magnitudes involved. In-order to take into consideration the 3-D effects in real time, the Level-1 flow model uses Fluid Model Interface (FMI) and corrects the aerodynamic coefficient data for airfoils at each station. One of the many advantages of this flow model is the ability to handle the data tables of various airfoils simultaneously and then pull out the data for the required airfoil at that instance. This simultaneous handling of multiple data tables helps us to simulate various active controls for the blades such as micro-tabs, deployable spoilers etc. in real time.

Chapter 3 presents us with a structural model developed upon the generalized Timoshenko beam theory which was developed by Dr. Otero [6,9]. The GTBM is a modification based upon the Variable Asymptotic Blade Sectional (VABS) model that was proposed by Prof. Hodges and his collaborators [10].The model thus generated which is able to work as a twisted and a curved composite beam also uses the same variables as the ones used by Timoshenko beam theory. However GTBM considerations doesn't keep the beam sections 
planar after deformation as is the case with Timoshenko beam theory. Instead of keeping the section planar, the real warping of the section is simulated by interpolating a 2-D finite element mesh. The geometrical complexity of the blade section coupled with the material inhomogeneity are then reduced to a stiffness matrix of a 1-D beam. This 1-D strain energy matrix is actually equivalent to the 3-D strain energy matrix. Eliminating the proposed kinematic assumptions generates a fully populated $6 \times 6$ symmetric matrix for the theoretical 1-D beam which consists of 21 independent variable instead of the original 6 fundamental stiffness variables of the Timoshenko beam theory [11]. This model, even for large displacements and rotations helps in precisely modeling the transverse shear and bending in two directions and also the torsion and extension of the blade structure which is accurately mapped as a 1-D finite element problem. The 3-D nonlinear anisotropic elasticity [L] problem is thus reduced into a linear 2-D cross sectional analysis.

Chapter 6 serves us with the analysis of all the numerical experimentation carried out during the span of research. The rotors blades used for these experimentations were based on 5-MW Reference Wind Turbine (RWT) project developed by NREL [12]. All the modifications on the blades are based on these blades as the stock design. Flexo-torsional effects as well as the material adaptivity was tested by altering the internal structure coupled with changes in materials that constituted the blade. A material hinge was also added on strategic geometric locations along the span of the blade to enhance the material adaptivness and provide us with an optimum power output. The outputs of a standard blade were then compared with various blades termed as super blades and also with blades having the newly designed material hinge. All these components are further used to computationally generate the next generation of upscaled wind turbines.

Finally in chapter 7 we will discuss the overall effect of the experiments done and also the future scope of this research work. 


\section{COMMON ODE FRAMEWORK}

A set of algorithms collectively known as the common ODE framework was designed to run a simultaneous analysis of the aeroelastic problem combined with any other control strategy that may be the need of time. This Common ODE framework thus designed are a series of computation codes that are implemented into a single and computationally selfadaptive algorithm which are developed using advanced numerical methods and are to be run with the help of HPC supercomputer. This article deals with tan overview of the capabilities of the Common ODE framework module and also the possibility of incorporating any control strategy change in the future.

As it will be described further 3 the structural model used for the computations is a Generalized Timoshenko Beam Model (GTBM). This model is used to effectively reduce the geometric complexity of the blade section along with material inhomogeneity if any into a stiffness matrix for an equivalent 1D beam. A non-linear adaptive type ODE solver is used to solve the equations of motion for this 1D beam problem obtained. The type of solver used works on variable time step as well as variable order ODE algorithm. This algorithm adapts itself by monitoring the local truncation error through each time step. The monitoring done thus helps in improving the efficiency and also ensuring the stability in time marching schemes.

The structural model is then subsequently combined with any of the three alternative flow models; each flow model vary from each other in the level of description and the amount of computational resources they require. The Figure 2.1 shows us a pseudo-code diagram of this interrelations between the dynamics of the structure, control systems, flow, and other devices. The use of these non-linear adaptive ODE algorithms provides us with the Common ODE framework with an ability to control the time dependent solutions for the aeroelastic problems.

As MATLAB provides with universality as well as the flexibility, the general structure of the code was scripted in MATLAB. This approach helped in easily creating the interactions among all the aspects of the common ODE framework along with the further amalgamation with the structural computations and solutions to the universal problems fur- 


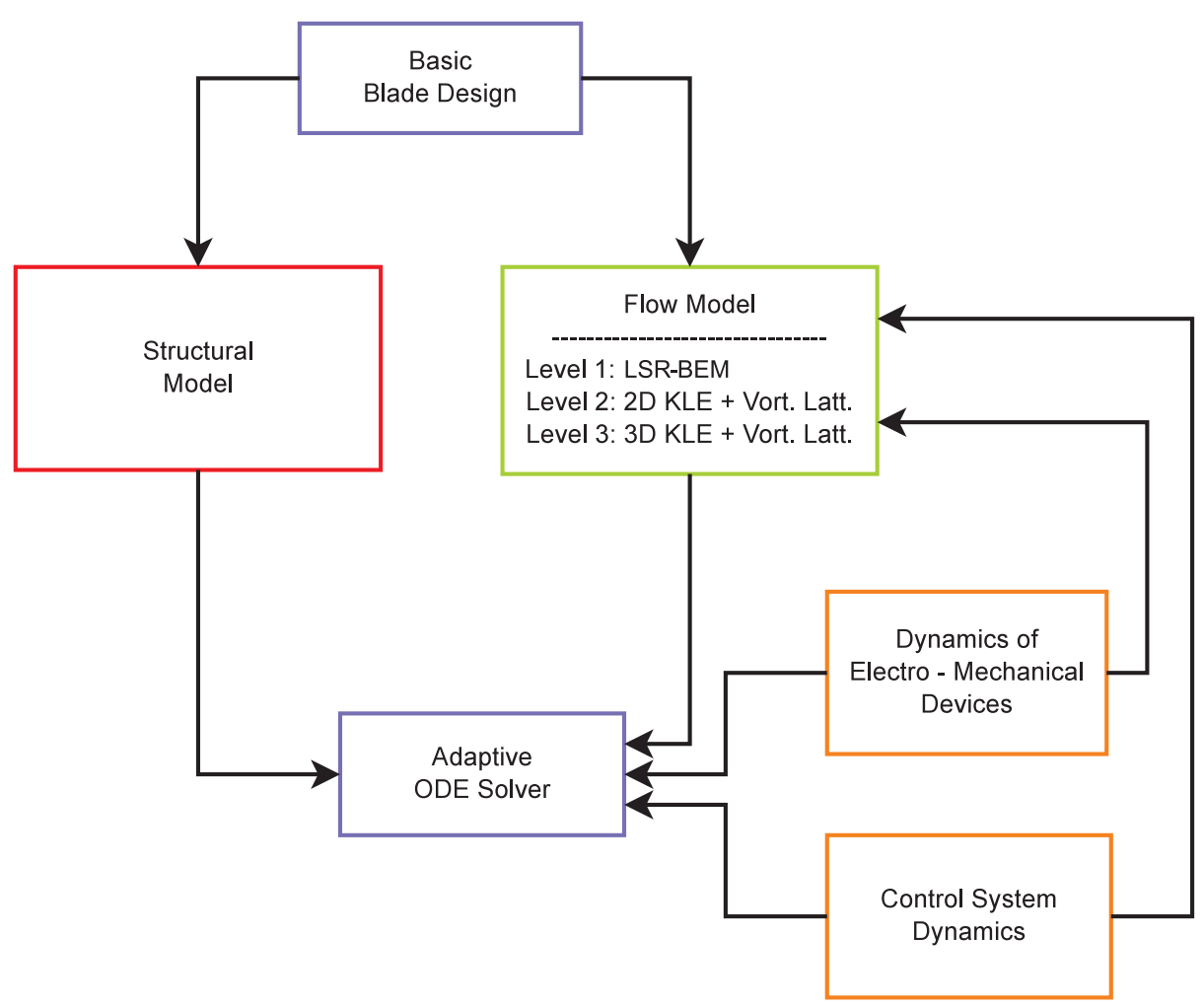

Figure 2.1. Pseudo-code diagram.

ther ahead.Instead of the orthodox monolithic design the modular approach significantly simplifies the process of code development. The primary advantage of it being the development and troubleshooting of the individual sub-models independently. This modular approach also helps in simultaneous analyses of the aeroelastic problem coupled with any novel control strategy

\subsection{Numerical implementation}

This article sheds some light on the numerical implementation of the defined Common ODE framework and how the non linear adaptive ODE protocol integrates different modules. Fig 2.2 shows us $n$ various computational models that are divided into three main blocks viz: Parameters input, Main Framework and Post-processing.

The problem at our disposal is defined in terms of simulation timespan cumulative with the amount of modules that intervene. The intervention of the modules is then identified and the data required for the evaluation of different instances of the ODE problem is then 
collected in this stage. The basic instances are divided into three major steps : Defining the initial conditions (IC), evaluations of the Right hand side (RHS), and lastly post-processing of the accepted time steps through the output function. In case of existence of the analytical form of the Jacobian matrix a feature to include the definition and evaluation of the Jacobian matrix for all the modules is made. This possibility helps to save a lot of computation time especially in cases, where the ODE code has to recalculate the Jacobian matrix incase if material stiffness arises $[13,14]$. Due to the absence of ability to compute the analytical solution for the Jacobian matrix in MATLAB we use the default numerical method in computation of the Jacobian matrix. The finite difference method used for computation increases the computation time by the factor of the square of present degrees of freedom [15].

The second block in the framework as seen in the Fig 2.2 is the actual ODE solver and sheds light on how the intervening modules are linked in the ODE framework. This is the Main Framework. After processing the information obtained from the Parameters-input block, the obtained parameters are distributed onto different sub-blocks for the corresponding computations. Now each sub block has a main function that helps in administering the already processed parameters to each of the designated modules and then the data from these modules is collected. The solution is collected in the form of one output function. The initial IC sub block will thus output a cumulative vector of input conditions to the common framework. This vector will contain the ICs of all the modules. The main RHS evaluator takes the IC vector and then distributes the IC elements to the corresponding modules and the RHS is solved for the current timestep. The ODE solver then accepts the solution for the current timestep after the RHS evaluation, the data collected can also be stored upon a hard disk drive. The data obtained as the solution is then reported back to different modules after it is processed by the output functions. The major advantage of this structure is that the whole process of computation is always monitored by the same self-adaptive ODE algorithm that monitors the local truncation error at each timestep which in turn is used to improve the efficiency and maintain the stability of the time marching scheme.

The third block exhibits the possibility of post-processing of the extracted data and generate reports at each time step for the variables involved. There is also a possibility to monitor other auxiliary variables rather than just the main variables. This capability aids to understand the interaction of various variables among the interacting physical mechanisms involved. 


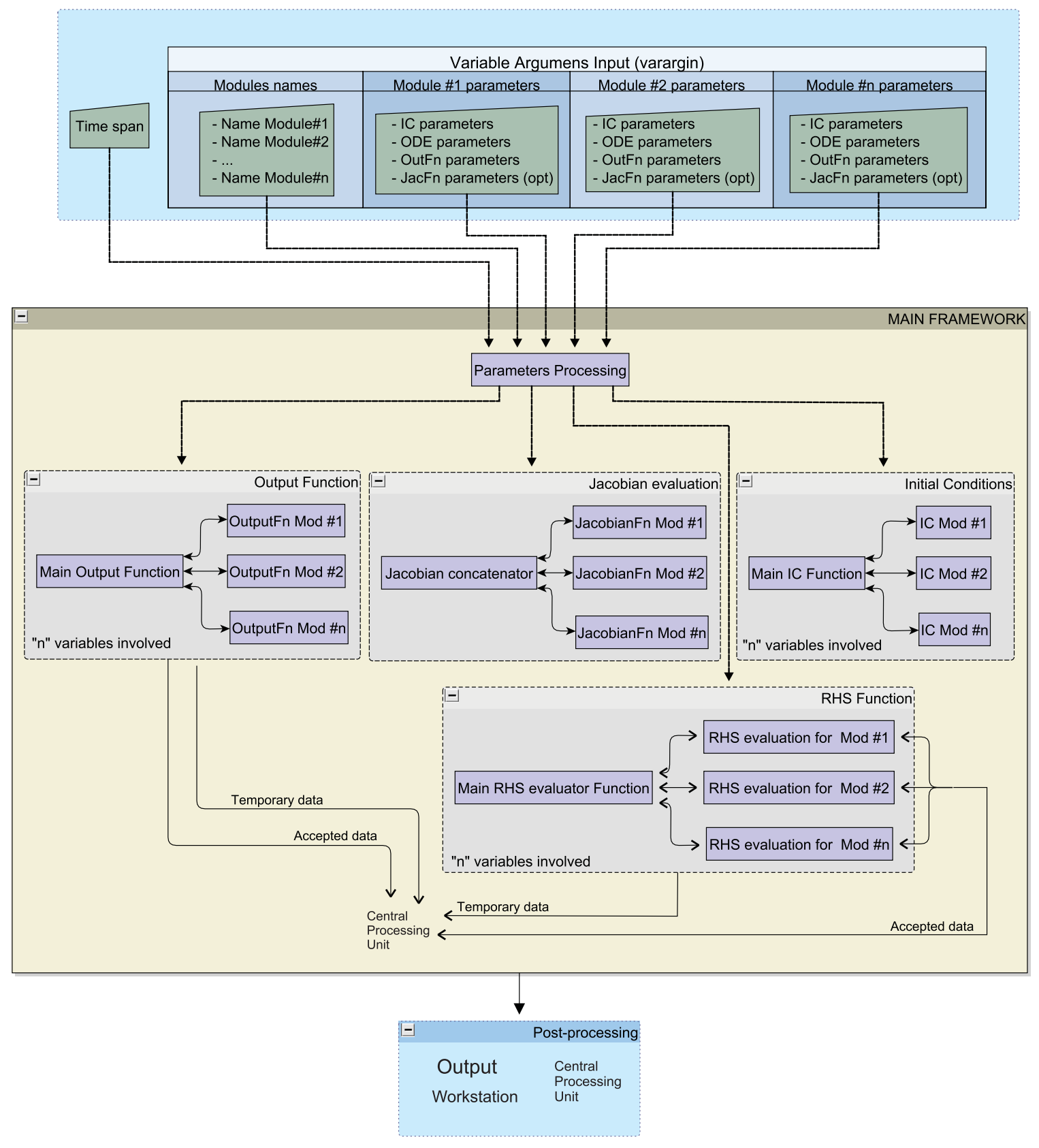

Figure 2.2. Common ODE framework. 


\subsection{The Level-1 flow model}

The flow model implemented in this study is the Level-1 flow model that involves modeling of the rotor wake aerodynamics with the aid of the Blade Element Momentum theory, further discussed in chapter 5. In future studies the next two flow models will be developed. These include a full simulation of the dynamics of the unsteady separated flow around the blade sections with the use of further generated adaptive algorithms which will be developed from the hybrid formulation of the Navier-Stokes equations.

The equations that model the dynamics of the control system as well as the electrochemical devices may also be added to the general ODE system. Any modifications in these control systems as well as the electrochemical devices will also affect the aeroelastic solution. This thus provides with a natural way to assimilate the dynamics of the control system into the structural response solution there by creating a general solution to the flow problem that is governed by the self-adaptive ODE algorithm. Integrating any further sub models of various physical phenomena thus remains a scope in the near future

Among the main modules shown in the Figure 2.1, the one which deals with the Basic Blade design not only does it provides us with the aerodynamic parameters for the normal operating conditions but it also provides us with the structural features that result from the blade section's internal layout, the material specifications, manufacturing procedures and also the flow control devices which may be further additions to the current configuration. Figure 2.3 provides us with a pseudo-code diagram with various sub routines in their respective modules and their interrelations. The structural model performing the dimensional reduction produces the ODE equations for the 1-D beam problem from the present 3-D blade structure. This dimensional reduction is one of the important characteristic of the structural module. The concentrated and the distributed forces that are required to evaluate the RHS of the ODE equations is obtained from the evaluation of the flow model and the control-system actuators The response from the electro-mechanical drive train provides us with the time-dependent value for the rotational speed of the main shaft which uses the torque from the rotor as the input. This value is also used to calculate the incoming flow on the blade sections as further explained in section 5.3.

The early stages of information about the structural response is provided by the linearization of the equations of the equivalent 1D beam problems and getting an aero-elastic steady state solution at comparatively low computational cost (see Fig. 2.3). The frequency as well as the linear vibrational modes of the deformed configuration can also be calculated by obtaining the solution for the Eigen-value problem of the linearized steady-state solution. These linear vibrational modes can further advantageously applied at the initial design stages of the blade structures as well as the control systems. Preliminary information about the structural response is provided, at relatively low computational cost, by linearizing the 
equations for the equivalent 1-D beam and getting an aeroelastic steady-state solution for a certain set of operational parameters (see Fig. 2.3). The frequencies and linear vibrational modes for that deformed configuration can also be obtained by solving an eigenvalue problem around that linearised steady-state solution. The latter is a valuable by-product of the dimensional-reduction technique that may be advantageously applied at the initial stages of the design of both the blade structure and the control system. 


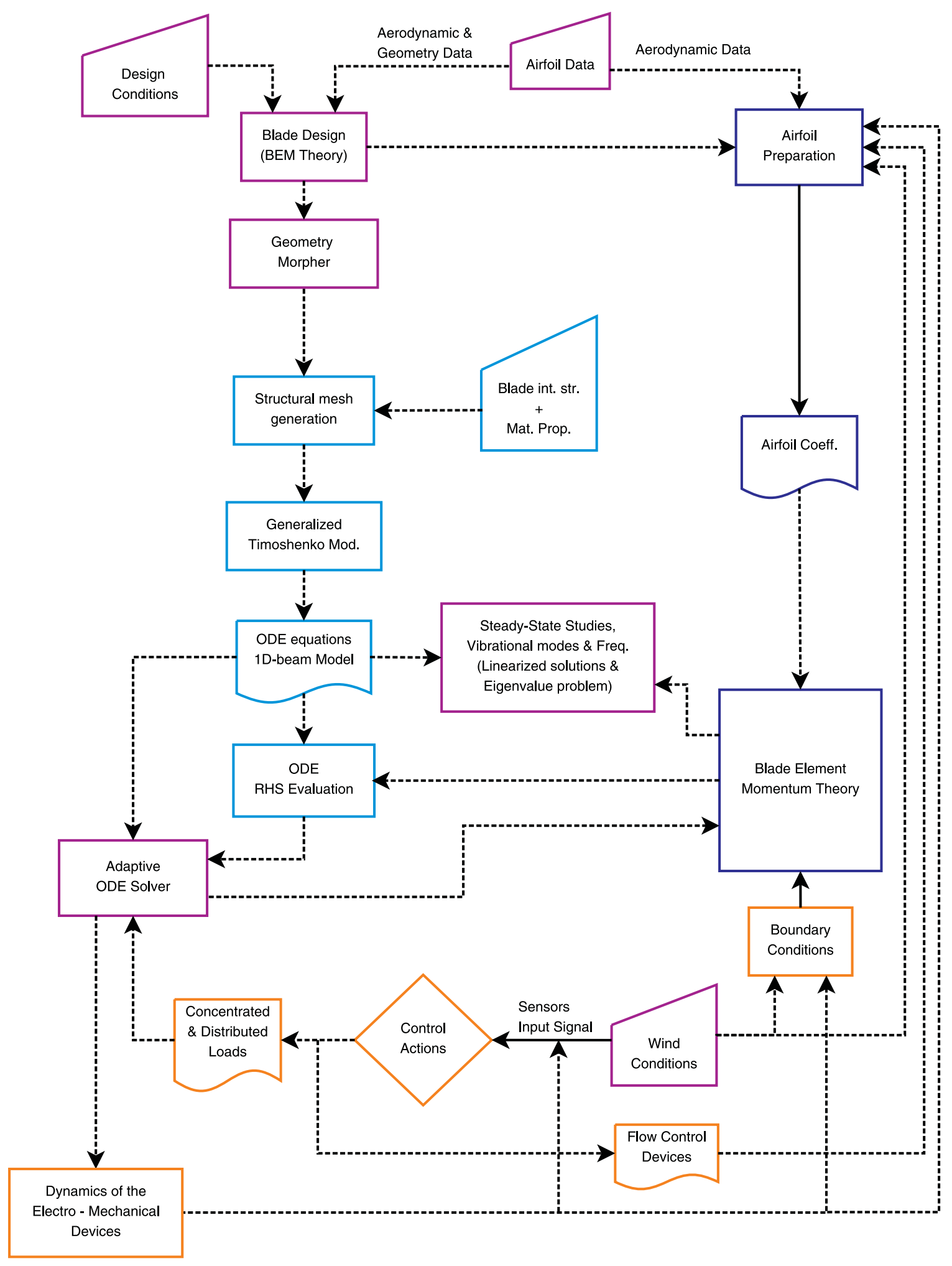

Figure 2.3. The Pseudo-code diagram corresponding to the common framework on the Level-1 flow model. 


\subsection{The stiff multistep BDF-ODE solver}

The adaptive stepsize technique is employed here to control the accuracy of the simulations and also to enhance their efficiency in providing a steady or a transient solution as the need may be. The adaptive time step algorithms generally determine the approximate values of the local truncation error to estimate the optimal time step. In a few standard algorithms, the accuracy requirement of the local truncation error is user defined. This value is then compared against the obtained values of the same which then should lie in the required accuracy range[16].

In this work, a BDF Backwards Differentiation formula solver with an adaptive stepsize solver is used. BDF ODE constantly monitors the rate of convergence [17]. If the code predicts that convergence for the current time step will not be achieved in the next 5 steps then the iteration process is halted as a result of which a new Jacobian is formed or a new integration step size is reduced. As the optimizations for reusing the Jacobians in BDFODE sizably reduce the time of recalculations, this solver is on par with other solvers [18] that deal with non-stiff problems.

We use a BDF solver that has adaptive stepsize control. We have adapted the ODE15s solver into the common ODE framework to compute the solutions to our aeroelastic problems.

The ODE15s is a variable order, variable step size implementation of the numerical differentiation formulae (NDF) in terms of backward differences [18] that also allows the integration using BDFs. The order of integration in ODE15s can be changed from 1 to 5 [18]. This provides us with an adaptability to expand its reach to various multiphysics situations. Being termed as the method with memory it is also well efficient in cases where the evaluation of the RHS of the ODE system is computationally taxing. The main intention behind using the ODE15s lies in its efficiency in dealing with the stiffness originated from the complex aeroelastic interactions. 


\section{The Structural Model}

The wind turbines are highly flexible structures. The complexity of the airfoil cross sections and the inhomogeneity in the material property distribution make them a difficult structure to be modeled. (see figure 3.1). . If the structural problem has to be solved along various timesteps then solving a detailed 2-Dmodel or a full 3-D solid model in that case will be highly expensive at the computation front. The kinematic assumptions done in classical theories like Bernoulli or the standard Timoshenko theory introduce significant computational errors especially in scenarios where the blade vibrates at a wavelength shorter than the length of the blade. This existing problem of modeling is made more intricate with the introduction of more complex blade geometry, new techniques in blade construction, and the use of new materials with an array of whole new properties.

Dr. Otero came up with the generalized Timoshenko code in order to deal with the fluid

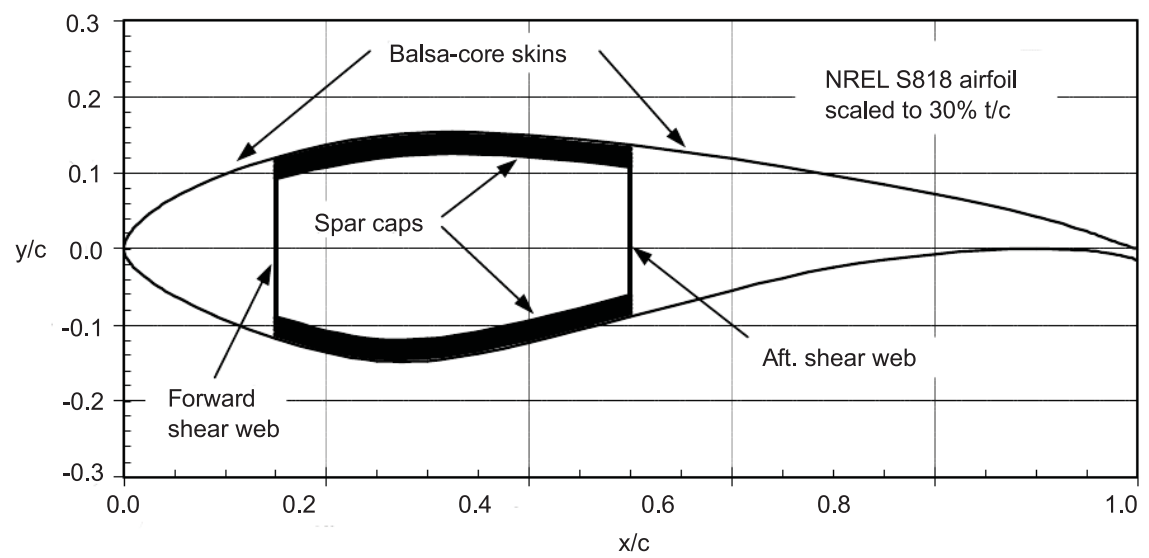

Figure 3.1. A schematic of blade structure architecture representing the current commercial design of the blade.The primary structural member is a box-spar, with a substantial build-up of spar cap material between the webs. The exterior skins and internal shear webs are both sandwich construction with triaxial fiberglass laminate separated by balsa core. (adapted from [19]). 
structure integration and the complex features of new-generation blades[6]. This generalized beam theory was modified on the basis of a model developed by Prof. Hodges and his collaborators[see 10, for example]. The generalized beam theory abandons the fact of the beam staying planar after the deformation. This assumption makes the theory to deal with the twisted composite materials using the same variables of the classical beam theory. Rather than keeping the section planar, the real warping of the deformed section is interpolated via a 2-D finite element mesh. The contribution of the deformation towards the stain energy is taken in as an input and put in terms of the 1-D Timoshenko variable which is carried out via pre-resolution. The material inhomogeneity and the geometrical complexities exhibited by these blades are further reduced into the stiffness matrix for the 1-D beam problem. The obtained 1-D strain energy is asymptotically equal to the actual 3-D strain energy. Eliminating all the kinematic assumptions made for mere practical purposes we obtain a fully populated $6 \times 6$ symmetric stiffness matrix for the 1 -D beam. The obtained matrix constitutes of almost 21 independent degrees of freedom as compared to the fundamental 6 degrees of freedom obtained via the classical Timoshenko beam theory[11].

In the event of large displacement or rotations of the beam section, the generalized model copes will with it allowing for an accurate modeling of the bending as well as the transverse shear in two directions with that being extension and torsion of the blade section under consideration mapped into an appropriate 1-D finite element problem. The 3-D nonlinear anisotropic elasticity problem is thus decomposed into a 2-D cross sectional analysis and a nonlinear 1-D beam problem. This global problem is required at each time step of a fluid structure interaction analysis. The 2-D cross-sectional analysis helps in calculating the 3-D warping function asymptotically which then further aids in constructing a constitutive 1-D model for non-linear beam analysis. The cross sectional analysis can be carried out in parallel for all the defined cross sections along the span of the blade. Once the global deformation is calculated via the 1-D beam analysis it can be then used to calculate the original 3-D fields like displacements, stresses, and strain. These variables are recovered using the already calculated warping functions.

\subsection{The Generalized Timoshenko Beam Model (GTBM)}

As seen in Fig 3.2 the beam is represented by a reference line, say $R$ in its undeformed state which can already be twisted and/or bent. At all points along $R$, are defined orthogonal triads $\underline{\mathbf{B}}_{1}, \underline{\mathbf{B}}_{2}, \underline{\mathbf{B}}_{3}$ which are also associated with that point. $\underline{\mathbf{B}}_{1}$, is the tangent to $R$ while $\underline{\mathbf{B}}_{2}, \underline{\mathbf{B}}_{3}$ lie on a plane which is normal to $R$. A corresponding coordinate sys-

tem $\left(X^{1}, X^{2}, X^{3}\right)$ is also defined where $X^{1}$ is the coordinate along $R$ while the rest two, $X^{2}, X^{3}$ are the Cartesian coordinates of the section plane under consideration.

After deformation the reference line $R$ observes a new orientation, denoted by $r$ and a 


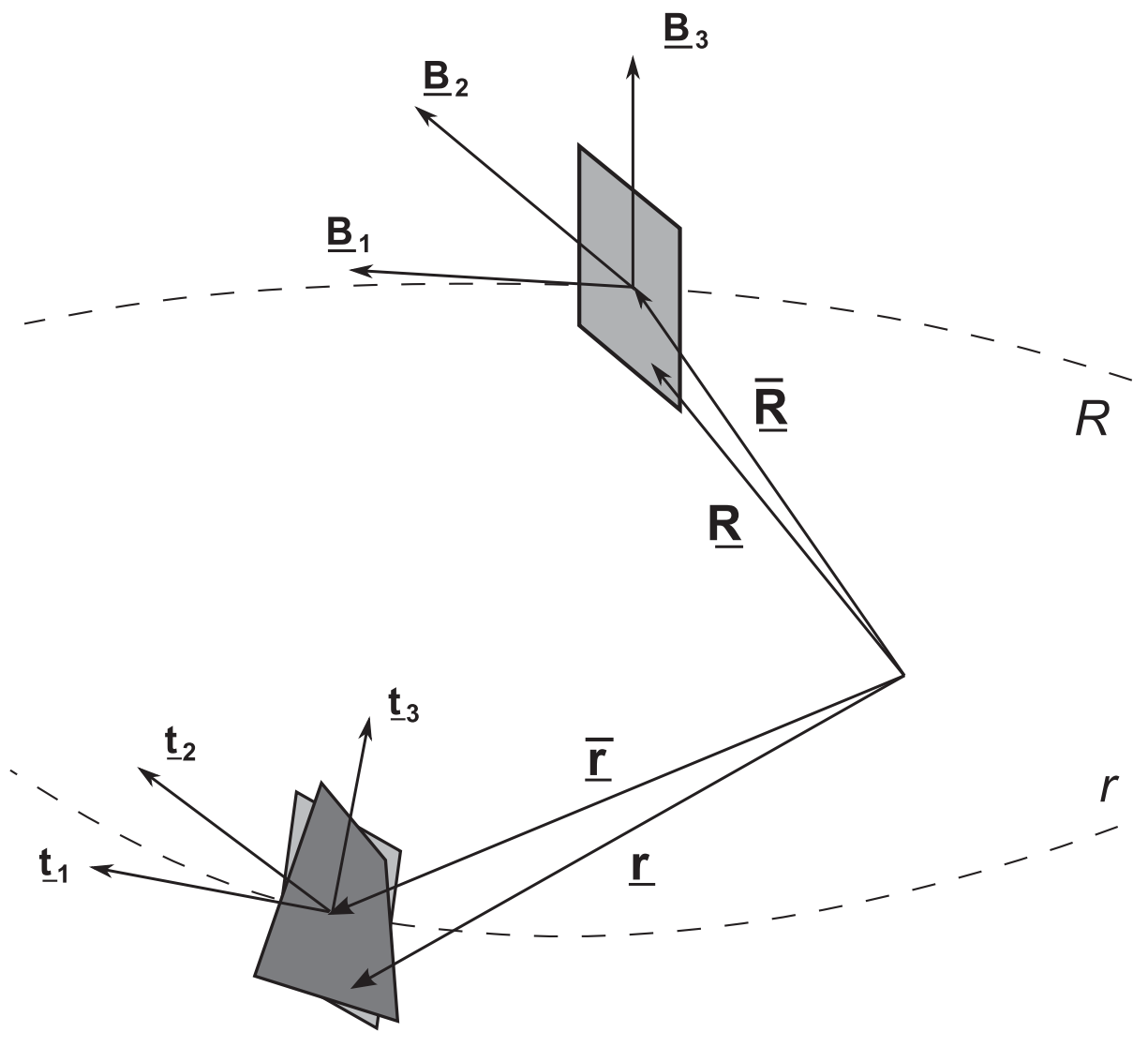

Figure 3.2. Explanation of the Generalized Timoshenko theory with a schematic showing the orthogonal triads,reference line, and beam pre and post-deformation. 
new triad is now associated with each point, $\underline{\mathbf{t}}_{1}, \underline{\mathbf{t}}_{2}, \underline{\mathbf{t}}_{3} . \underline{\mathbf{t}}_{1}$ here is the tangent to $r$ at that very point while $\underline{\mathbf{t}}_{2}, \underline{\mathbf{t}}_{3}$ lie on the normal plane. The spatial position of any generic point lying on the section plane can be given by

The position of a generic point on each section in the underformed configuration may be written as

$$
\underline{\mathbf{R}}\left(X^{i}\right)=\underline{\overline{\mathbf{R}}}\left(X^{1}\right)+X^{\alpha} \underline{\mathbf{B}}_{\alpha}\left(X^{1}\right)
$$

Where $\underline{\mathbf{R}}$ is the positon of the center of curvature along the reference line. The index $\alpha$ assumes the values 2 and 3. Also Einstein notations are followed here where in the same index undergoes summation. The position vector of the material point originally defined by $\underline{\mathbf{R}}\left(X^{i}\right)$ in deformed state is defined by the vector $\underline{\mathbf{r}}\left(X^{i}\right)$ after deformation.

$$
\underline{\mathbf{r}}\left(X^{i}\right)=\underline{\overline{\mathbf{R}}}+\underline{\mathbf{u}}+X^{\alpha} \underline{\mathbf{t}}_{\alpha}+w_{i} \underline{\mathbf{t}}_{i},
$$

where $w_{i}$ is the contribution of warping towards the displacement. The components of gradient of deformation tensor can now be formulated as $F_{i j}=\underline{\mathbf{t}}_{i} \cdot \mathbf{g}_{k} \underline{\mathbf{G}}^{k} \cdot \underline{\mathbf{B}}_{j}$, where $\mathbf{g}_{k}$ and $\underline{\mathbf{G}}^{k}$ are the covariant base vectors of the deformed configuration and undeformed configuration respectively. The kinematic description of equations (3.1) and (3.2) help in obtaining these covariant vectors. A measure for the 3D strain is provided by the JaumannBiot-Cauchy strain tensor and can be written as $\Gamma_{i j}=\frac{1}{2}\left(F_{i j}+F_{j i}\right)-\delta_{i j}$. The measure is provided in form of beam strain measures and arbitrary warping functions. The strain energy function can then be calculated as

$$
2 U=\left\langle\left\langle\Gamma^{T} \mathcal{S} \Gamma\right\rangle\right\rangle,
$$

$\mathcal{S}$ is the characteristics of the material represented in to $\underline{\mathbf{B}}_{i}$ coordinates $\langle\langle\bullet\rangle\rangle=$ $\int_{s} \bullet \sqrt{G} d X^{2} d X^{3}$ where $s$ implies the integration of the given function over the cross section while $\mathrm{G}$ being the determinant of the matrix in its undeformed base.

This is then followed by the derivation of the strain energy expression that is asymptotically correct up to the second order of $h / l$ and $h / R_{0}$. h here is the characteristic size of the section, $l$ is the characteristic wavelength of the deformation along the axis of the beam while $R_{0}$ are the characteristic radii of all the initial curvatures and twists in the beam. Usually a complete second order strain energy equation is sufficient for the construction of a generalized Timoshenko model. As accepted generally the shear strain measures are an order lower than that in the classical beam theory, the second order strain energy is sufficient for computation [11]. The Variational Asymptotic Method proposed in [20] is successfully used to asymptotically approximate the 3-D strain energy upto the second order. The complete derivation which is available in[21] is used to obtain the strain energy expression expressed as 


$$
2 U=\varepsilon^{T} \mathbf{A} \varepsilon+\varepsilon^{T} 2 \mathbf{B} \varepsilon^{\prime}+\varepsilon^{\prime T} \mathbf{C} \varepsilon^{\prime}+\varepsilon^{T} 2 \mathbf{D} \varepsilon^{\prime \prime},
$$

A, B, C, D here are the matrices that contain the information of the geometry as well as the material properties of the considering cross section area. ()$^{\prime}$ is the partial derivative with respect to the axial coordinate $X^{1}$, while $\varepsilon=\left[\begin{array}{llll}\bar{\gamma}_{11} & \bar{\kappa}_{1} & \bar{\kappa}_{2} & \bar{\kappa}_{3}\end{array}\right]^{T}$ are the strain measures defined in the classical Bernoulli beam theory: The extension of beam reference line here is $\bar{\gamma}_{11} \cdot \bar{\kappa}_{1}$ is the torsion while $\bar{\kappa}_{2}$ and $\bar{\kappa}_{3}$ are the bending of the reference line in axes 2 , and 3 due to the caused deformation.

To obtain the matrices in the equations (3.4), the variational asymptotic approach used requires the discretization of the warping functions $w_{i}$ (3.2) using the finite element techniques. Four constraints are then imposed on $w_{i} .\left\langle w_{i}\right\rangle=0$ and $\left\langle X^{2} w_{3}-X^{3} w_{2}\right\rangle=0$, where $\langle\bullet\rangle=\int_{s} \bullet d X^{2} d X^{3}$. These four constraints are used to eliminate the four rigid modes of displacements of the warped sections. These four modes being the three linear DOF and rotation around $\underline{\mathbf{t}}_{1}$ ). They are already included in the Bernoulli strain measures $\varepsilon$.

Dr. Otero uses the classical Lagrangian-multiplier technique to impose the already discussed constraints and to solve the present expanded system for the constrained variational problem. The whole solution is thus reduced to a single step. The simplification carried out cuts considerably on the computational cost but also allows us to use the internal node condensation technique as the finite element discretization technique. With the use of tri-quadrilateral finite element technique the efficiency of our solution is substantially improved.

Even though the expression (3.4) is asymptotically correct, it is difficult to be put in practice due to the presence of derivatives of the classical strain measures that require complicated boundary conditions for exact problem definition. The Timoshenko theory on the other hand is free of such drawbacks. Instinctively the next step for here is to fit expression (3.4) into a Generalized Timoshenko form.

$$
2 U=\left[\begin{array}{ll}
\boldsymbol{\epsilon}^{T} & \boldsymbol{\gamma}_{s}^{T}
\end{array}\right]\left[\begin{array}{cc}
\mathbf{X} & \mathbf{Y} \\
\mathbf{Y}^{T} & \mathbf{G}
\end{array}\right]\left[\begin{array}{c}
\boldsymbol{\epsilon} \\
\boldsymbol{\gamma}_{s}
\end{array}\right]=\boldsymbol{\epsilon}^{T} \mathbf{X} \boldsymbol{\epsilon}+2 \boldsymbol{\epsilon}^{T} \mathbf{Y} \boldsymbol{\gamma}_{s}+\boldsymbol{\gamma}_{s}^{T} \mathbf{G} \boldsymbol{\gamma}_{s}
$$

Where $\boldsymbol{\epsilon}=\left[\begin{array}{llll}\gamma_{11} & \kappa_{1} & \kappa_{2} & \kappa_{3}\end{array}\right]^{T}$ are the classical Timoshenko strain measures as a result of extension, torsion and bending. $\gamma_{s}=\left[\begin{array}{lll}2 \gamma_{12} & 2 \gamma_{13}\end{array}\right]^{T}$ are the transverse shear strains. $\mathrm{X}, \mathrm{Y}$ and $\mathrm{G}$ are obtained in such a way that the strain energy in (3.4) is equal to that obtained in (3.5) at least up to the second order.[see 6, for more detail].

Lastly, a stiffness matrix is so obtained for the 1-D beam problem by reordering the 
matrix $\left[\begin{array}{cc}\mathbf{X} & \mathbf{Y} \\ \mathbf{Y}^{T} & \mathbf{G}\end{array}\right]$, in a way that helps us get a functional for the strain energy density of espression (3.5)

$$
2 U=\bar{\gamma}^{T} \overline{\mathbb{S}} \bar{\gamma}
$$

$\bar{\gamma}=\left[\begin{array}{l}\gamma \\ \kappa\end{array}\right]$, here is a more conveniently organized array of the Timoshenko measures of deformation. $\boldsymbol{\gamma}^{T}=\left[\begin{array}{lll}\gamma_{11} & 2 \gamma_{12} & 2 \gamma_{13}\end{array}\right]$ and $\boldsymbol{\kappa}^{T}=\left[\begin{array}{lll}\kappa_{1} & \kappa_{2} & \kappa_{3}\end{array}\right]$.

The tri-quadrilateral finite element technique was used to obtain the convergence in the 2-D sections. This technique is based on the use of a nine-node biquadriatic isoparametric finite elements. Such elements show a high convergence rate and also provide us with an additional ability of reducing the skin error versus the linear elements due the virtue of their biquadriatic interpolation of geometric coordinates. More details in BatheBathe [22].

The tri-quadrilateral isoparametric elements implemented help us get the best of the both worlds. They combine the advantages of convergence of the nine node quadrilateral isoparametric elements with geometric ability of the triangular elements to replicate the geometry more accurately than the quads. For the formation of a nine node tri-quadrilateral element we divide each standard triangular element into three quadrilaterals. The static condensation of the nodes lying inside each triangle reduces the time for computation significantly by reducing the number of nodes. The values of these internal nodes is then extracted from the solution on the non-condensable nodes. The internal nodes are expressed in terms of nodes that lie on the elemental boundary by following the classical procedure of elemental condensation[22]. This process of condensation helps us reduce the size of the new system by an approximate of $40 \%$ of the original system. The use of static condensation procedure reduces the size of stiffness matrices that arise from finite as well as spectral element methods. This procedure also ultimately improves the condition number of the final condensed system. More details in Ponta [23]

\subsection{The 1D model}

The intrinsic equations for the beam from the variational principles are used to solve the one dimensional beam problem of the equivalent beam [24] while being weighted in an energy-consistent method according to Patil and Althoff Patil and Althoff [25], producing the following variational equation 


$$
\begin{array}{r}
\int_{0}^{\ell}[\underbrace{\delta \overline{\mathbf{V}}^{T} \overline{\mathbb{I}} \dot{\overline{\mathbf{V}}}}_{1}+\underbrace{\delta \overline{\mathbf{F}}^{T} \overline{\mathbb{S}}^{-1} \dot{\overline{\mathbf{F}}}}_{2}] d X^{1}=\int_{0}^{\ell}[\underbrace{\delta \overline{\mathbf{V}}^{T} \overline{\mathbf{E}}^{\prime}}_{3}+\underbrace{\delta \overline{\mathbf{V}}^{T} \hat{\mathbf{K}} \overline{\mathbf{F}}}_{4}+\underbrace{\delta \overline{\mathbf{V}}^{T} \hat{\gamma} \overline{\mathbf{F}}}_{5}+ \\
\underbrace{\delta \overline{\mathbf{V}}^{T} \overline{\mathbf{f}}}_{6}-\underbrace{\delta \overline{\mathbf{V}}^{T} \hat{\mathbf{V}} \overline{\mathbf{I}} \overline{\mathbf{V}}}_{7}+\underbrace{\delta \overline{\mathbf{F}}^{T} \overline{\mathbf{V}}^{\prime}}_{8}-\underbrace{\delta \overline{\mathbf{F}}^{T} \hat{\mathbf{K}}^{T} \overline{\mathbf{V}}}_{9}-\underbrace{\delta \overline{\mathbf{F}}^{T} \hat{\boldsymbol{\gamma}}^{T} \overline{\mathbf{V}}}_{10}] d X^{1},
\end{array}
$$

where

$$
\begin{array}{cc}
\overline{\mathbf{F}}=\left[\begin{array}{c}
\mathbf{F} \\
\mathbf{M}
\end{array}\right], \quad \overline{\mathbf{V}}=\left[\begin{array}{c}
\mathbf{V} \\
\boldsymbol{\Omega}
\end{array}\right], \quad \overline{\mathbf{f}}=\left[\begin{array}{c}
\mathbf{f} \\
\mathbf{m}
\end{array}\right], \\
\hat{\gamma}=\left[\begin{array}{cc}
\tilde{\boldsymbol{\kappa}} & \mathbf{0} \\
\tilde{\gamma} & \tilde{\boldsymbol{\kappa}}
\end{array}\right], \quad \hat{\mathbf{V}}=\left[\begin{array}{cc}
\tilde{\Omega} & \mathbf{0} \\
\tilde{\mathbf{V}} & \tilde{\Omega}
\end{array}\right], \quad \hat{\mathbf{K}}=\left[\begin{array}{cc}
\tilde{\mathbf{K}} & \mathbf{0} \\
\tilde{\mathbf{e}_{1}} & \tilde{\mathbf{K}}
\end{array}\right] .
\end{array}
$$

The tilde indicates askew-symmetric matrix linked to a vector magnitude. For example say we have an arbitrary pair of vectors $\mathbf{A}$ and $\mathbf{B}$ then the matrix vector product of $\tilde{\mathbf{A}} \mathbf{B}$ is equivalent to $\mathbf{A} \times \mathbf{B}$. Hence $\tilde{\gamma}$ is linked to $\gamma, \tilde{\kappa}$ is linked to $\kappa$ so on. Thus the matrix $\hat{\gamma}$ is just the rearrangement of the strain measures vector $\bar{\gamma}$ as defined earlier in this chapter. The generalized velocity vector $\overline{\mathbf{V}}$ denotes the linear velocity while the matrix $\hat{\mathbf{V}}$ denotes the components of angular velocity. The $\hat{\mathbf{K}}$ matrix reflects the initial torsion and curvatures of the beam. The generalized-forces vector $\tilde{\mathbf{e}}_{1}$ stores the forces and the moments of the strain measures $\left(\mathbf{e}_{1}^{T}=\left[\begin{array}{lll}1 & 0 & 0\end{array}\right]\right.$, the unit vector being $\left.X^{1}\right) . \overline{\mathbf{F}}$ is the generalized-distributedloads vector and it represents the forces and moments that are distributed along the axis of the beam. $\overline{\mathbb{S}}$ here is the stiffness matrix for the one dimensional model corresponding to the one in equation (3.6). $\overline{\mathbb{I}}$ is the inertia of each section. A dot above indicates the time derivative while a prime indicates the derivative with respect to the longitudinal coordinate of the beam $X^{1}$.

The variational formulation in discussion is discretized by the spectral-element methods(see [26,27]). The magnitudes in (3.7) are thus replaced by their interpolated counterparts. The interpolation function array can be expressed as: $\overline{\mathbf{V}}=\mathbf{H}_{\overline{\mathbf{V}}}^{e} \mathbf{Q}^{e}$, and $\overline{\mathbf{F}}=\mathbf{H}_{\overline{\mathbf{F}}}^{e} \mathbf{Q}^{e}$, where $\mathbf{H}_{\overline{\mathbf{V}}}^{e}$ and $\mathbf{H}_{\overline{\mathbf{F}}}^{e}$ are the interpolation-function arrays. $\mathbf{Q}^{e}$ is a vector that contains the nodal values of the generalized velocities and generalized forces. The subscript e implies the discretization of the concerned term at an elemental level. These terms then vanish when the matrices are assembled together to form a global matrix representing the whole beam. $\overline{\mathbf{V}}^{\prime}=\mathbf{B}_{\overline{\mathbf{V}}}^{e} \mathbf{Q}^{e}$, and $\overline{\mathbf{F}}^{\prime}=\mathbf{B}_{\overline{\mathbf{F}}}^{e} \mathbf{Q}^{e}$, are the axial derivatives of the magnitudes which are also interpolated on the same lines with $\mathbf{B}_{\overline{\mathbf{V}}}^{e}$ and $\mathbf{B}_{\overline{\mathbf{F}}}^{e}$ as the interpolation arrays.

$$
\delta \mathbf{Q}^{e T} \mathbf{M}_{\mathbf{1}}^{e} \dot{\mathbf{Q}}^{e}=\delta \mathbf{Q}^{e T}\left(\mathbf{K}_{\mathbf{1}}^{e}+\mathbf{K}_{\mathbf{2}}^{e}\right) \mathbf{Q}^{e}+\delta \mathbf{Q}^{e T} \mathbf{K}_{\mathbf{q}}^{e} \overline{\mathbf{q}}^{e}+\delta \mathbf{Q}^{e T} \mathbf{B}_{Q}^{e}\left(\mathbf{Q}^{e}\right)
$$


where

$$
\begin{aligned}
& \mathbf{M}_{\mathbf{1}}^{e}=\int_{-1}^{1}\left[\mathbf{H}_{\overline{\mathbf{V}}}^{e T} \overline{\mathbb{I}} \mathbf{H}_{\overline{\mathbf{V}}}^{e}+\mathbf{H}_{\overline{\mathbf{F}}}^{e^{T}} \overline{\mathbb{S}}^{-1} \mathbf{H}_{\overline{\mathbf{F}}}^{e}\right] J d t, \\
& \mathbf{K}_{\mathbf{1}}^{e}=\int_{-1}^{1}\left[\mathbf{H}_{\overline{\mathbf{V}}}^{e T} \mathbf{B}_{\overline{\mathbf{F}}}^{e}+\mathbf{H}_{\overline{\mathbf{F}}}^{e T} \mathbf{B}_{\overline{\mathbf{V}}}^{e}\right] J d t \mathbf{Q}^{e}, \\
& \mathbf{K}_{\mathbf{2}}^{e}=\int_{-1}^{1}\left[\mathbf{H}_{\overline{\mathbf{V}}}^{e T} \hat{\mathbf{K}} \mathbf{H}_{\overline{\mathbf{F}}}^{e}-\mathbf{H}_{\overline{\mathbf{F}}}^{e T} \hat{\mathbf{K}}^{T} \mathbf{H}_{\overline{\mathbf{V}}}^{e}\right] J d t, \\
& \mathbf{K}_{\mathbf{q}}^{e}=\int_{-1}^{1} \mathbf{H}_{\overline{\mathbf{V}}}^{e T} \mathbf{H}_{\overline{\mathbf{F}}}^{e} J d t .
\end{aligned}
$$

$\mathbf{M}_{1}^{e}$ corresponds to the elemental discretization of the terms 1 and 2 providing us with a corresponding mass matrix. $\mathbf{K}_{1}^{e}$ corresponds to terms 3 and 8 and represents the stiffness matrix of the 1-D beam problem. $\mathbf{K}_{2}^{e}$ corresponds to the terms 4 and 9 and represents additional stiffness in relation with the twist and the curvature in the undeformed configuration. $\mathbf{K}_{\mathbf{q}}^{e}$ corresponds to the terms 6 and contributes towards the distributed loads. $t$ is the natural coordinate among the elements. $J$ is the Jacobian for mapping the problem coordinates form $X^{1}$ to $t$ coordinates (see [22]). The discretized version of (3.7) related to all non-linear interactions, terms 5,7,and 10 leaves us with

$$
\mathbf{B}_{Q}^{e}\left(\mathbf{Q}^{e}\right)=\int_{-1}^{1}\left[\mathbf{H}_{\overline{\mathbf{V}}}^{e}{ }^{T} \hat{\gamma} \mathbf{H}_{\overline{\mathbf{F}}}^{e}-\mathbf{H}_{\overline{\mathbf{V}}}^{e T} \hat{\mathbf{V}} \overline{\mathbb{I}} \mathbf{H}_{\overline{\mathbf{V}}}^{e}-\mathbf{H}_{\overline{\mathbf{F}}}^{e T} \hat{\gamma}^{T} \mathbf{H}_{\overline{\mathbf{V}}}^{e}\right] \mathbf{Q}^{e} J d t
$$

The differential equation obtained after assembling all the elemental terms into a global system is as follows:

After the assembly of the elemental terms into the global system, we obtain the differential equations for the solution of the 1-D nonlinear problem of the equivalent beam mentioned in section 2.1:

$$
\dot{\mathbf{Q}}=\mathbf{M}_{1}^{-1}\left[\left(\mathbf{K}_{\mathbf{1}}+\mathbf{K}_{\mathbf{2}}\right) \mathbf{Q}+\mathbf{K}_{\mathbf{q}} \overline{\mathbf{q}}+\mathbf{B}_{Q}(\mathbf{Q})\right]
$$

This is the solution of the 1-D nonlinear problem for the equivalent beam as mentioned ion 2.1 All the frequencies and the linear vibrational modes around the steady state can obtained with linearizing the equation (3.9). the linearization of of any arbitrary nonlinear term $\mathbf{B}_{Q}^{e}\left(\mathbf{Q}^{e}\right)$ around any given configuration say $\mathbf{Q}_{1}^{e}$ produces the matrix 


$$
\begin{aligned}
\mathbf{K}_{\mathbf{N}}^{e}\left(\mathbf{Q}_{1}^{e}\right)= & \int_{-1}^{1}\left\{\mathbf{H}_{\overline{\mathbf{V}}}^{e} T\left[\hat{\gamma}_{1} \mathbf{H}_{\overline{\mathbf{F}}}^{e}-\hat{\mathbf{V}}_{1} \overline{\mathbb{I}} \mathbf{H}_{\overline{\mathbf{V}}}^{e}-\hat{\mathbf{F}}_{1} \overline{\mathbb{S}}^{-1} \mathbf{H}_{\overline{\mathbf{F}}}^{e}+\hat{\mathbf{P}}_{1} \mathbf{H}_{\overline{\mathbf{V}}}^{e}\right]+\right. \\
& \left.\mathbf{H}_{\overline{\mathbf{F}}}^{e T}\left[\hat{\mathbf{V}}_{1}^{T} \overline{\mathbb{S}}^{-1} \mathbf{H}_{\overline{\mathbf{F}}}^{e}-\hat{\gamma}_{1}^{T} \mathbf{H}_{\overline{\mathbf{V}}}^{e}\right]\right\} J d t
\end{aligned}
$$

where

$$
\hat{\mathbf{F}}=\left[\begin{array}{cc}
0 & \tilde{\mathbf{F}} \\
\tilde{\mathbf{F}} & \tilde{\mathbf{M}}
\end{array}\right], \quad \hat{\mathbf{P}}=\left[\begin{array}{cc}
0 & \tilde{\mathbf{P}}_{v} \\
\tilde{\mathbf{P}}_{v} & \tilde{\mathbf{P}}_{\omega}
\end{array}\right]
$$

Matrix $\hat{\mathbf{F}}$ on similar lines of what has been discussed earlier is mere rearrangement of the components of the generalized force vectors $\overline{\mathbf{F}}$ as it is defined above. Matrix $\hat{\mathbf{P}}$ is also a rearrangement of the components of the generalized momentum vector given by $\overline{\mathbf{P}}=\left[\begin{array}{l}\mathbf{P}_{v} \\ \mathbf{P}_{\omega}\end{array}\right]$. It represents all the linear as well as the angular momenta related with the generalized velocities $(\overline{\mathbf{P}}=\overline{\mathbb{I}} \overline{\mathbf{V}})$. The subscript 1 indicates the value of the magnitude at any given state $\mathbf{Q}_{1}^{e}$

The solution to the nonlinear problem at disposal was obtained after assembling all the element level terms into a global system. The solution to (3.7) was obtained by iteratively solving the equation for $\Delta \mathrm{Q}$

$$
\left[\mathbf{K}_{\mathbf{1}}+\mathbf{K}_{\mathbf{2}}+\mathbf{K}_{\mathbf{N}}\left(\mathbf{Q}^{(i)}\right)\right] \Delta \mathbf{Q}=-\mathbf{K}_{\mathbf{q}} \overline{\mathbf{q}}-\left(\mathbf{K}_{\mathbf{1}}+\mathbf{K}_{\mathbf{2}}\right) \mathbf{Q}^{(i)}-\mathbf{B}_{Q}\left(\mathbf{Q}^{(i)}\right),
$$

The global vector for the nodal values of all the generalized velocities and forces can be represented as : $\mathrm{Q}^{(i+1)}=\mathbf{Q}^{(i)}+\Delta \mathrm{Q}$.

We obtain the vibrational modes of the blade structure from the steady state solution. The corresponding natural frequencies to theses nodes are obtained by solving the eigenvalue problem for the same

$$
\mathbf{M}_{\mathbf{1}} \dot{\mathbf{Q}}+\left[\mathbf{K}_{\mathbf{1}}+\mathbf{K}_{\mathbf{2}}+\mathbf{K}_{\mathbf{N}}\left(\mathbf{Q}^{(i)}\right)\right] \mathbf{Q}=\mathbf{0} .
$$

The results of these intrinsic equations lead to the displacements and rotations of the blade sections. These values are recovered by using the kinematic equations for the beam (see [10]) 


$$
\begin{aligned}
\mathbf{u}^{\prime}-\mathbf{C}_{r R}^{T}\left(\boldsymbol{\gamma}+\mathbf{e}_{1}\right)+\mathbf{e}_{1}+\tilde{\mathbf{K}} \mathbf{u} & =\mathbf{0}, \\
\tilde{\mathbf{K}}+\tilde{\boldsymbol{\kappa}}+\mathbf{C}_{r R}^{\prime} \mathbf{C}_{r R}^{T}-\mathbf{C}_{r R} \tilde{\mathbf{K}} \mathbf{C}_{r R}^{T} & =\mathbf{0},
\end{aligned}
$$

$\mathbf{u}$ here is the displacement vector for each point along the reference line point from its position in the undeformed reference configuration to the corresponding point in the deformed configuration. $\mathbf{C}_{r R}$ is the rotation matrix to rotate the original triad from its undeformed configuration into the deformed one with both defined as a function of the longitudinal coordinate $X^{1}$. The strains $\gamma$ and $\kappa$ are calculated from the generalized forces and the stiffness of the corresponding blade sections. Equations 3.12 and 3.13 are also linearized as well as discretized using the spectral element method

\subsection{Constitutive relations of composite materials}

Composite materials exhibit nonlinear stress-strain relationships as a result of the behavior of differently combined materials under load. However in scenarios with small deformations these composite materials show a behavior similar to that of a Hookean material. Keeping in mind the scope of this thesis it is convenient to assume that the materials are not given a chance to reach the state of failure. The materials in this thesis keeping in mind the assumption just made will always act in the linear-elastic domain. This behavior will help to model the required materials before reaching a failure due to delamination or fiber failure

These materials working under the linear-elastic domain can further be classified into three categories viz. orthotropic, transversely isotropic, or linear isotropic materials. Toying with the fiber orientation during the manufacturing period lets one to design material properties as required. Specific structure properties can thus be obtained and there transformation into different co-ordinate system is studied(see [28-31]). This chapter helps in briefly understanding the constitutive relations that define each of the above mentioned material categories. For every given second order strain tensor $\underline{\boldsymbol{e}}$ and a tensor of stress say $\underline{\boldsymbol{s}}$, for every material there will also exist a fourth order tensor by the name constitutive tensor $\mathcal{S}$.

$$
\underline{\boldsymbol{s}}=\underline{\mathcal{S}}: \underline{\boldsymbol{e}},
$$

Which in its component form looks like :

$$
s_{i j}=\mathcal{S}_{i j k l} e_{k l} \text {. }
$$


A general second order tensor is defined by nine independent constants and a fourth order tensor has eighty one independent constants in its definition. The stress and strain tensors being symmetric are defined by just six independent constants instead of nine. The presence of this fact makes the quadratic form of the energy deformation tensor ; $\underline{\boldsymbol{e}}: \underline{\boldsymbol{s}}$ to exhibit the following symmetry at the component level for any given base.

$$
\mathcal{S}_{i j k l}=\mathcal{S}_{j i k l}=\mathcal{S}_{i j l k}=\mathcal{S}_{k l i j}
$$

This reduces the number of independent constants to a low of twenty one in most general cases.

The equation 3.14 , by the virtue of the symmetry exhibited can be simply written as

$$
\mathrm{s}=\mathcal{S e}
$$

With the arrays in 3.17 expressed as

$$
\mathbf{s}=\left[\begin{array}{c}
s_{11} \\
s_{22} \\
s_{33} \\
s_{23} \\
s_{31} \\
s_{12}
\end{array}\right], \mathcal{S}=\left[\begin{array}{llllll}
\mathcal{S}_{1111} & \mathcal{S}_{1122} & \mathcal{S}_{1133} & \mathcal{S}_{1123} & \mathcal{S}_{1131} & \mathcal{S}_{1112} \\
\mathcal{S}_{1122} & \mathcal{S}_{2222} & \mathcal{S}_{2233} & \mathcal{S}_{2223} & \mathcal{S}_{2231} & \mathcal{S}_{2212} \\
\mathcal{S}_{1133} & \mathcal{S}_{2233} & \mathcal{S}_{3333} & \mathcal{S}_{3323} & \mathcal{S}_{3331} & \mathcal{S}_{3312} \\
\mathcal{S}_{1123} & \mathcal{S}_{2223} & \mathcal{S}_{3323} & \mathcal{S}_{2323} & \mathcal{S}_{2331} & \mathcal{S}_{2312} \\
\mathcal{S}_{1131} & \mathcal{S}_{2231} & \mathcal{S}_{3331} & \mathcal{S}_{2331} & \mathcal{S}_{3131} & \mathcal{S}_{3112} \\
\mathcal{S}_{1112} & \mathcal{S}_{2212} & \mathcal{S}_{3312} & \mathcal{S}_{2312} & \mathcal{S}_{3112} & \mathcal{S}_{1212}
\end{array}\right] \quad \mathbf{e}=\left[\begin{array}{c}
e_{11} \\
e_{22} \\
e_{33} \\
2 e_{23} \\
2 e_{31} \\
2 e_{12}
\end{array}\right]
$$

$\mathcal{S}$ is called the stiffness matric while its inverse $\mathcal{C}$ is called the compliance matrix. Mechanical properties of a material like the modulus of elasticity, Poisson's ratio and shear modulus as a function of the components of matrices $\mathcal{C}$ and $\mathcal{S}$ can be used to make the equations of the compliance matrix even more compact. Thus it is more convenient to find the compliance matrix first and then invert it inorder to obtain the Stiffness matrix as and when required.

\section{Orthotropic materials}

These materials have three main planes of symmetry. The properties of orthotropic materials are different along each axis but are uniform along a single axis. These materials are anisotropic. Any stress applied perpendicular to any plane of symmetry does not generate any shear stress. This kind of materials are of great interest for the wind turbine 
manufacturers.

$$
\begin{gathered}
\mathcal{S}=\left[\begin{array}{cccccc}
\mathcal{S}_{x x x x} & \mathcal{S}_{x x y y} & \mathcal{S}_{x x z z} & 0 & 0 & 0 \\
\mathcal{S}_{x x y y} & \mathcal{S}_{y y y y} & \mathcal{S}_{y y z z} & 0 & 0 & 0 \\
\mathcal{S}_{x x z z} & \mathcal{S}_{y y z z} & \mathcal{S}_{z z z z} & 0 & 0 & 0 \\
0 & 0 & 0 & \mathcal{S}_{y z y z} & 0 & 0 \\
0 & 0 & 0 & 0 & \mathcal{S}_{z x z x} & 0 \\
0 & 0 & 0 & 0 & 0 & \mathcal{S}_{x y x y}
\end{array}\right] . \\
\mathcal{C}=\left[\begin{array}{cccccc}
\frac{1}{E_{x}} & -\frac{\nu_{y x}}{E_{y}} & -\frac{\nu_{z x}}{E_{z}} & 0 & 0 & 0 \\
-\frac{\nu_{x y}}{E_{x}} & \frac{1}{E_{y}} & -\frac{\nu_{z y}}{E_{z}} & 0 & 0 & 0 \\
-\frac{\nu_{x z}}{E_{x}} & -\frac{\nu_{y z}}{E_{y}} & \frac{1}{E_{z}} & 0 & 0 & 0 \\
0 & 0 & 0 & 1 & 0 & 0 \\
0 & 0 & 0 & 0 & \frac{1}{G_{z x}} & 0 \\
0 & 0 & 0 & 0 & 0 & \frac{1}{G_{x y}}
\end{array}\right]
\end{gathered}
$$

Following identities can be identified form the symmetry of the compliance matrix:

$$
\frac{\nu_{y x}}{E_{y}}=\frac{\nu_{x y}}{E_{x}}, \quad \frac{\nu_{z x}}{E_{z}}=\frac{\nu_{x z}}{E_{x}}, \quad \frac{\nu_{z y}}{E_{z}}=\frac{\nu_{y z}}{E_{y}},
$$

It can be clearly seen that an orthotropic material can be defined by nine independent constants; three shear moduli, three elasticity moduli and three out of the six available Poisson's coefficients

The stiffness matrix can also be described with the help of these nine constants, however computing the inverse of the compliance matrix from equation 3.20 is more convenient.

\section{Transversely isotropic materials}

Transversely isotropic materials have just one axis of symmetry. The best example of this is the honeycomb type of materials that are used in the manufacturing of sandwich composites. The fibers are oriented in the same axis as that of the axis of symmetry in such laminates.

The equation to the stiffness matrix of these materials at the component level is given as (for further details see [31]) Matrix $\mathcal{S}$, from equation 3.17, has the same expression as for orthotropic materials. Due to the restrictive symmetry, this type of materials could be

characterized through five independent coefficients: $\alpha, \beta, \gamma, \lambda$ and $G$ (for further details 
see [31]) such that the components of the constitutive tensor can be expresses as:

$$
\begin{aligned}
\mathcal{S}_{i j k l}= & \lambda \delta_{i j} \delta_{k l}+G\left(\delta_{i k} \delta_{j l}+\delta_{i l} \delta_{j k}\right)+\alpha\left(\delta_{i j} a_{k} a_{l}+\delta_{k l} a_{i} a_{j}\right) \\
& +\beta\left(\delta_{i l} a_{k} a_{j}+\delta_{j k} a_{i} a_{l}+\delta_{i k} a_{j} a_{l}+\delta_{j l} a_{i} a_{k}\right)+\gamma\left(a_{i} a_{j} a_{k} a_{l}\right),
\end{aligned}
$$

The material is classified with the help of five independent coefficients given by $\alpha, \beta, \gamma$, $\lambda$ and $G$. The restrictive symmetry exhibited by such materials and also with the help of identities already identified in equation 3.21 we can write where $\delta_{i j}$ and $\underline{\mathbf{a}}=a_{i} \underline{\mathbf{B}}_{i}$ is the Kroneker delta and the direction of the symmetric axis expressed in $\underline{\mathbf{B}}_{i}$ vector base.

$$
E_{z}=E_{y}, \quad \nu_{z x}=\nu_{y x}, \quad G_{y z}=\frac{E_{y}}{2\left(1+\nu_{z y}\right)}, \quad G_{x z}=G_{x y} .
$$

The compliance matrix for transversely isotropic materials is thus written as

$$
\mathcal{C}=\left[\begin{array}{cccccc}
\frac{1}{E_{x}} & -\frac{\nu_{y x}}{E_{y}} & -\frac{\nu_{y x}}{E_{y}} & 0 & 0 & 0 \\
-\frac{\nu_{y x}}{E_{y}} & \frac{1}{E_{y}} & -\frac{\nu_{z y}}{E_{y}} & 0 & 0 & 0 \\
-\frac{\nu_{y x}}{E_{y}} & -\frac{\nu_{z y}}{E_{y}} & \frac{1}{E_{y}} & 0 & 0 & 0 \\
0 & 0 & 0 & \frac{2\left(1+\nu_{z y}\right)}{E_{y}} & 0 & 0 \\
0 & 0 & 0 & 0 & \frac{1}{G_{x y}} & 0 \\
0 & 0 & 0 & 0 & 0 & \frac{1}{G_{x y}}
\end{array}\right]
$$

One can very well obtain the corresponding stiffness matrix by inverting the above matrix as discussed earlier.

\section{Isotropic materials}

Materials of this kind are usually defined by five material constants Young modulus $(E)$, the shear modulus $(G)$, the volumetric modulus $(K)$, the Poisson coefficient $(\nu)$ and the Lamé coefficient $\lambda$ ) which happen to define the components of the constitutive vector. These constants are not independent and two are required to define the other three[28]. The correlations can be seen as follows: 


$$
\begin{aligned}
\lambda & =\frac{\nu E}{(1+\nu)(1-2 \nu)}=\frac{2 \nu G}{1-2 \nu}, \\
K & =\frac{E}{3(1-2 \nu)}=\lambda+\frac{2}{3} G, \\
G & =\frac{E}{2(1+\nu)}, \\
E & =\frac{(3 \lambda+2 G)}{(\lambda+G)} G, \\
\nu & =\frac{\lambda}{2(\lambda+G)} .
\end{aligned}
$$

The stiffness and the compliance matrices for such materials can be written as below :

$$
\begin{gathered}
\mathcal{S}=\left[\begin{array}{ccccccc}
\frac{E(1-\nu)}{(1+\nu)(1-2 \nu)} & \frac{E \nu}{(1+\nu)(1-2 \nu)} & \frac{E \nu}{(1+\nu)(1-2 \nu)} & 0 & 0 & 0 \\
\frac{E \nu}{(1+\nu)(1-2 \nu)} & \frac{E(1-\nu)}{(1+\nu)(1-2 \nu)} & \frac{E \nu}{(1+\nu)(1-2 \nu)} & 0 & 0 & 0 \\
\frac{E \nu}{(1+\nu)(1-2 \nu)} & \frac{E \nu}{(1+\nu)(1-2 \nu)} & \frac{E(1-\nu)}{(1+\nu)(1-2 \nu)} & 0 & 0 & 0 \\
0 & 0 & 0 & \frac{E}{2(1+\nu)} & 0 & 0 \\
0 & 0 & 0 & 0 & \frac{E}{2(1+\nu)} & 0 \\
0 & 0 & 0 & 0 & 0 & \frac{E}{2(1+\nu)}
\end{array}\right], \\
\mathcal{C}=\left[\begin{array}{cccccc}
\frac{1}{E} & -\frac{\nu}{E} & -\frac{\nu}{E} & 0 & 0 & 0 \\
-\frac{\nu}{E} & \frac{1}{E} & -\frac{\nu}{E} & 0 & 0 & 0 \\
-\frac{\nu}{E} & -\frac{\nu}{E} & \frac{1}{E} & 0 & 0 & 0 \\
0 & 0 & 0 & \frac{1}{G} & 0 & 0 \\
0 & 0 & 0 & 0 & \frac{1}{G} & 0 \\
0 & 0 & 0 & 0 & 0 & \frac{1}{G}
\end{array}\right]
\end{gathered}
$$

\section{Change of basis of the stiffness and compliance matrices}

The components of the constitutive tensor are the building blocks of the stiffness and compliance vectors. These components are expressed in relation to with the material structure such as axis of symmetry, planes of symmetry etc. The matrix components may have to be reoriented by the change of basis operations to make the materials comply with the material requirement to the ones defined in the structural problem. The change of basis op- 
eration which is carried out on these matrices is same as the one that was discussed,however the matrices are rotated instead of being reprojected. Rotation matrices are used here.

The constitutive tensor in its component form $\mathcal{S}_{i j k l}$ which is originally expressed in the base $\underline{\mathbf{b}}_{p}$ can be expressed as $\hat{\mathcal{S}}_{a b c d}$ in the changed base $\underline{\hat{\mathbf{b}}}_{q}$ :

$$
\hat{\mathcal{S}}_{a b c d}=\mathcal{S}_{i j k l} \hat{C}_{i a} \hat{C}_{j b} \hat{C}_{k c} \hat{C}_{l d}
$$

Where $\hat{C}_{m n}$ are the director cosines rotating the tensor from $\underline{\mathbf{b}}_{p}$ to base $\underline{\mathbf{b}}_{q}$ so that :

$$
\hat{\mathbf{b}}_{q}=\hat{C}_{q p} \underline{\mathbf{b}}_{p} .
$$

This operation could be done in two ways. The first one is by operating directly with the components of the constitutive tensor and the linear operators as described in equation 3.27. The second is to create a unique matrix to do the analogous operations directly on the stiffness matrix. In this work we chose to implement the first option.

The laminates are generally built out of various layers which may or may not differ in materials and/or orientation. A laminate is usually defined by two terns. The $\underline{\mathbf{r}}_{i}$ tern is the first of the characteristics that defines the orientation of the constitutive tensor. The numbers 1,2 , and 3 are usually replaced by the letters $l, n$, and $t$ to represent the three axes viz longitudinal, normal, and tangential respectively (i.e. $\underline{\mathbf{r}}_{1}=\underline{\mathbf{e}}_{l}, \underline{\mathbf{r}}_{2}=\underline{\mathbf{e}}_{t}$ and $\underline{\mathbf{r}}_{3}=\underline{\mathbf{e}}_{n}$ ). $\mathbf{y}_{i}$ on the other hand is the tern that defines the laminate. $\mathbf{y}_{1}$ is the laminate direction, $\mathbf{y}_{2}$ is perpendicular to $\mathbf{y}_{1}$ while $\mathbf{y}_{3}$ is the direction perpendicular to the laminate. $\mathbf{y}_{3}$ is parallel to $\underline{\mathbf{r}}_{3}$. Each layer is thus defined in terms of rotaions in the magnitude of $\theta_{3}$ around the base vector $\mathbf{y}_{3}$.

$[0,45,60,-30]$ implies that the laminate is made up of four layers oriented at the angles $0,45,60$, and ấL $\breve{S} 30$ degrees. A few other forms of denoting the laminates are : $\left[0,45,60_{2},-30\right]=[0,45,60,60,-30],[0,45]_{3}=[0,45,0,45,0,45],[0,45]_{s}=$ $[0,45,45,0]$ the subscript s here denotes symmetry.

For each blade section the vector $\mathbf{y}_{i}$ is represents to the reference coordinate system. The vector $\underline{\mathbf{y}}_{1}$ is parallel to $\underline{\mathbf{B}}_{1}$ which is perpendicular to the blade section. $\mathbf{y}_{2}$ is a tangent to the laminate and makes an angle of $\theta_{1}$ with $\underline{\mathbf{B}}_{2}$

The rotation of the constitutive matrix is done prior to the defining of the laminate as $\theta_{3}$ is a material property. The lamination angle over the blade section on the contrary depends solely on the angle of its application. The coordinate transformation happens in two steps with the first being the rotation about $\underline{\mathbf{r}}_{3}$ by an angle of $\theta_{3}$. Second is the rotation around $\mathbf{y}_{i}$ through an angle of magnitude $\theta_{1}$ to finally represent the constitutive tensor in the $\underline{\mathbf{B}}_{i}$ coor- 
dinate system. Only after these transformations is the stiffness matrix calculated 3.18.The orientation of different coordinate systems with the layers and the laminate can be seen in Figure 3.3

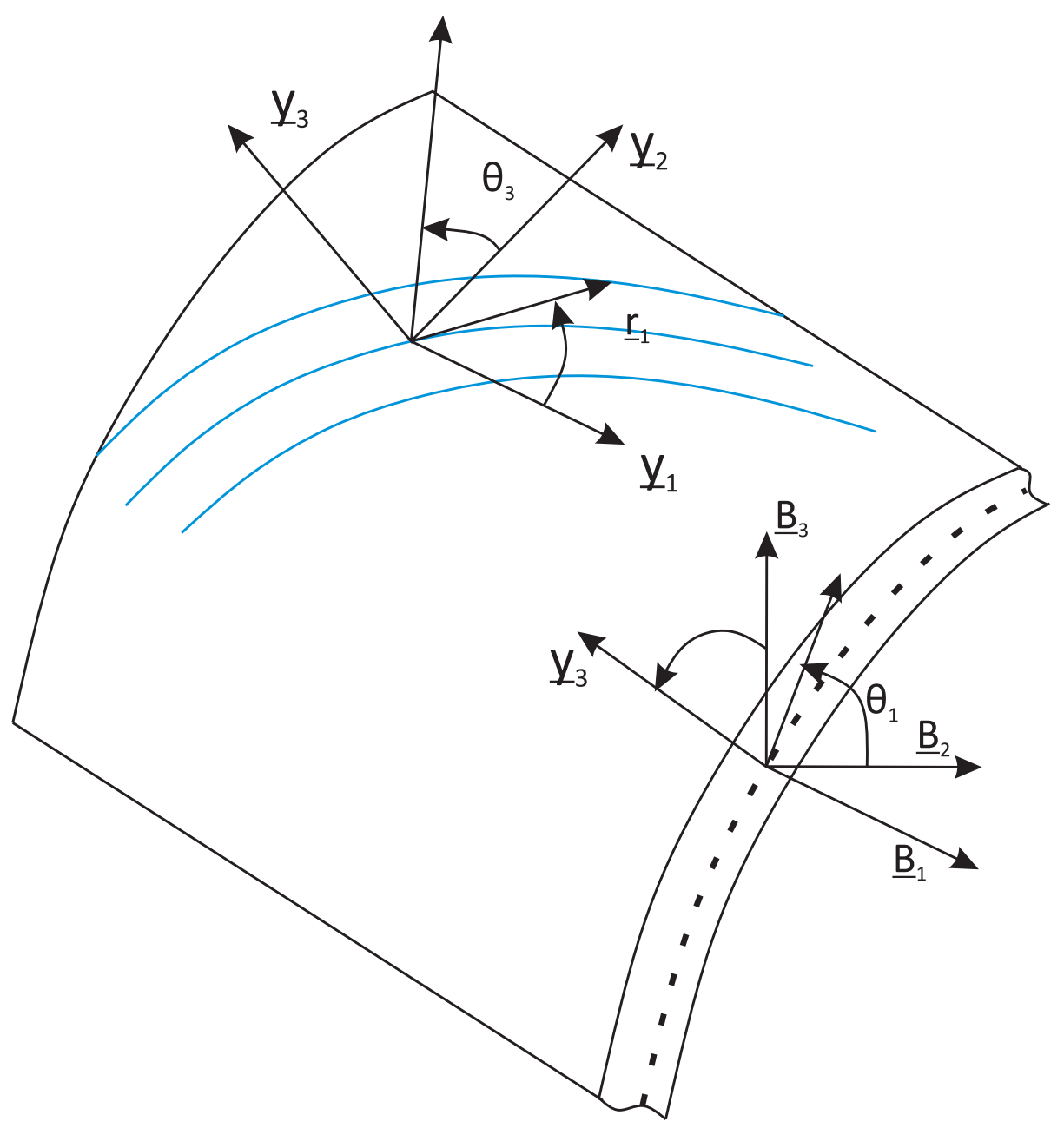

Figure 3.3. The reference frames for a laminate and their orientations. (Adapted from [9]) 


\section{INTERNAL BLADE ARCHITECTURE}

As seen in chapter 4 the blade section was divided into 7 parts in order to address each of them specifically as they differ in properties both materially as well as structurally. The first part of the blade was the nose followed by two spar caps. The spar caps were then added upon by the shear webs and lastly the tail was designed. As described in Chapter 4 article 4.3 specific structural properties can be extracted by tweaking the fiber orientation during the manufacturing stage itself. This division of the whole blade into seven parts helped the blade specifically catering the structural needs of each region of the blade rather than generating one monolithic component with near zero control over structural adaptivity. Since all the materials in this thesis were considered to act only in the linear elastic domain failure of these materials would only be a result of fiber failure or failure due to de-lamination.

\subsection{Blade Section Structural Design}

During the span of working on materially adaptive wind turbine blades there was an inception of an idea under which the whole blade was divided into 9 parts instead of 7 . Doing this provided a higher level of flexibility in designing the blades. The manufacturing angles were provided an extra degree of freedom while designing the upper and the lower part of the blade. The fiber orientations add up to the stiffness of the blade sections. The orientations of these fibers and the way of denoting them are explained well in 4.3 The process of changing this internal structure was started by redefining the airfoils _ geo and being renamed as airfoils_ Boxbeam_ geo. The Box in the name denotes the box like structure created by the sparcaps and the shear webs. A further step in this would designing blade sections of various internal architechture. Blades with D-Spar configuration where in just a single sparcap assuming the shape of letter $\mathrm{D}$ with a single shear web forms the major internal structure of the blade is under research. Following that will be an I-beam. This configuration will club both shear webs into one at the center of the sparcaps creating an I-beam. 


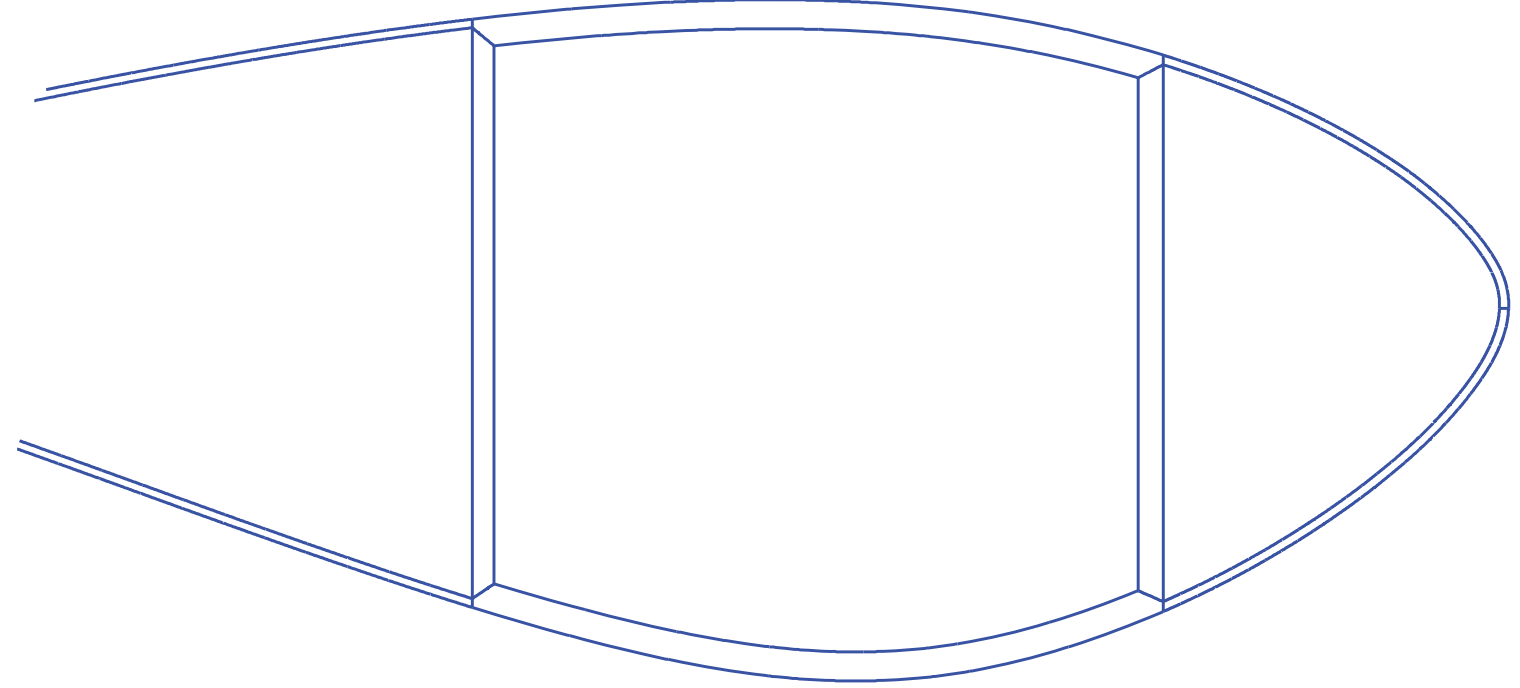

Figure 4.1. A concept of D-Spar internal configuration.

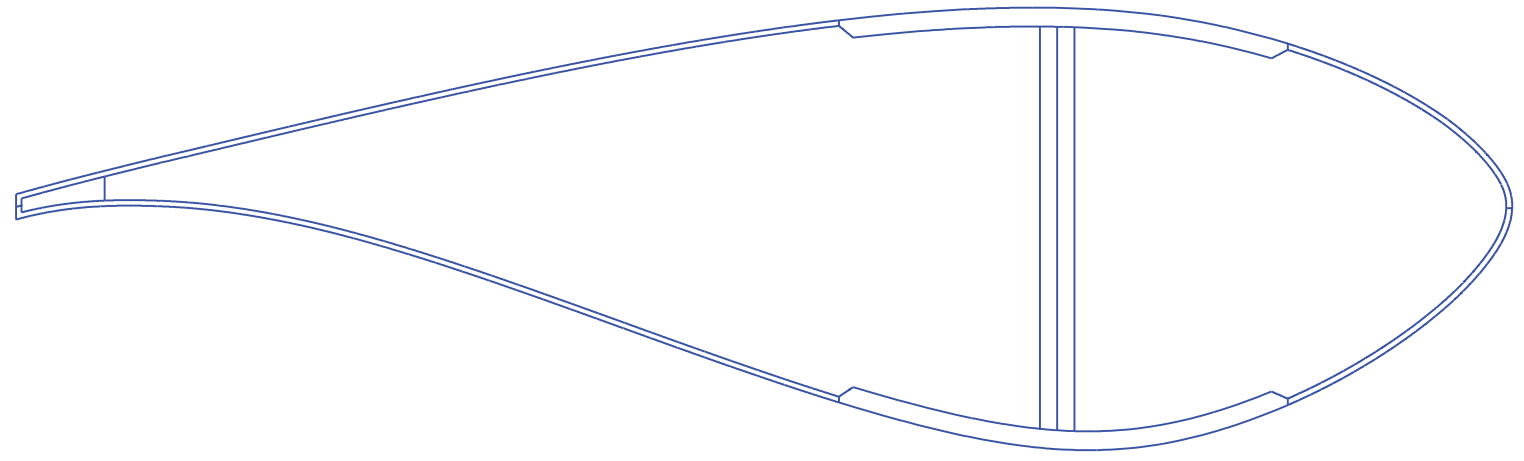

Figure 4.2. A concept of I-Beam internal configuration.

\subsubsection{External Spline}

The routine begins with describing the exterior spline of the shell structure of the blade section. This spline is the one that is obtained by the coordinates that describe the airfoil. These co-ordinates are available from the public domain. The airfoil described by this routine corresponds with that specified at the station under consideration. The whole blade along its span was divided into 19 stations thereby requiring the Boxbeam_ geo to run nineteen times. 


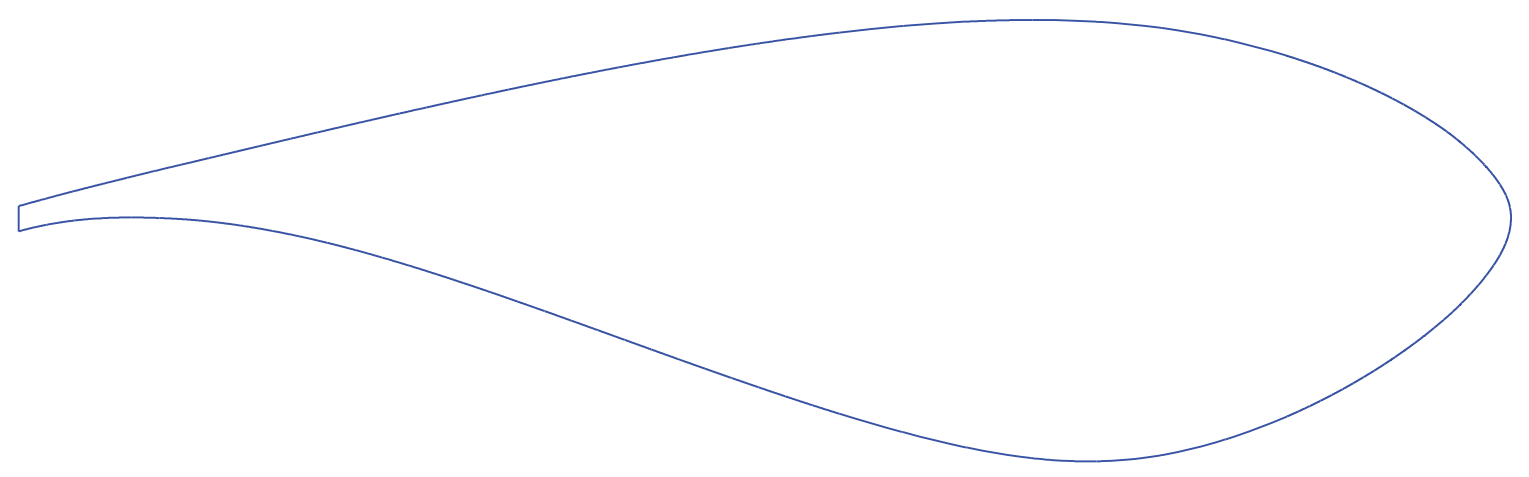

Figure 4.3. First step in creation of the airfoil External Airfoil Creation.

\subsubsection{Nose Structure}

Once the external shell spline was completely designed we then focused our attention on the sectioning the external spline and creating the internal structure. The internal spline was first extracted as just an offset of the external spline created. Various splines of the structure were defined by the help of indices which helped to map the offset spline for just the part specified. The external spline was also divided in same manner as that of the internal spline to generate sections. As per the nose layer thickness the first spline to be extracted was the top half of the nose shell which started from the leading edge to shear location one. This was the location which marked the beginning of the first shear web. The figure below shows us the spline that is created.

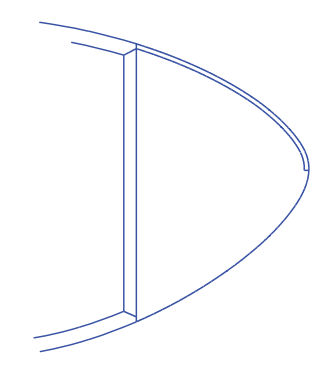

Figure 4.4. Internal structure of the airfoil showing the split in the Nose structure (Upper Half).

Similarly the spline describing the inner wall of the nose spans from the leading edge to shear location one however this time in the negative y direction. 


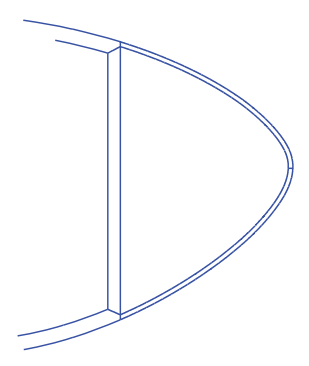

Figure 4.5. Internal structure of the airfoil showing the split in the Nose structure.

\subsubsection{Sparcap Design}

After defining the nose we then went forward in defining the sparcaps. Following the same protocol as the one followed while designing the inner walls of the blade first the inner wall of the top spar cap was described followed by describing the lower spar cap. Ind 3 and Ind 4 were the two indices that helped in mapping this spline. These indices spanned between the position of the first shear web and the second shear web. Suitable tolerance was given to the indices in order to seek the point closest to the locations they were designed to function to avoid any discontinuity in the airfoil surface on the inner side due to the creation of these sections.

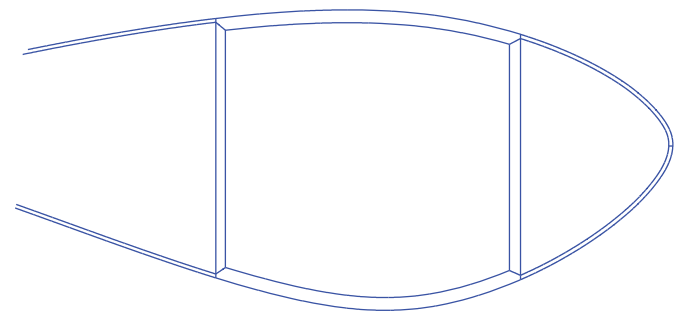

Figure 4.6. Internal structure of the airfoil showing the sparcap structure.

\subsubsection{Tail Design}

Following the sparcaps was the tail section of the airfoil which was also designed in 2 parts to incorporate the new design of the airfoil. Special care was required while desgining this section of the blade. The whole blade spanned for $61.5 \mathrm{~m}$ and was divided into 19 stations. The airfoil at each section had a different shape by the virtue of transitioning form a cylinder at the root to a perfect airfoil as we went along the span. As the shape of these airfoils changed the main effect was seen at trailing edge with drastic changes in the shape of the airfoil towards the tail. The airfoils under considerations were open airfoils, 
the routine was written in such a way that it would check the proximity of the first point of the airfoil with the last one if the points were close enough the code would just collapse the points midway. However there were airfoils in that did exhibit a larger gap between these two checkpoints. A suitable tolerance value was thus decided and added to the code. Whenever the points under check showed a distance larger than the tolerance then instead of collapsing onto a single point a small segment joining these two points was created.

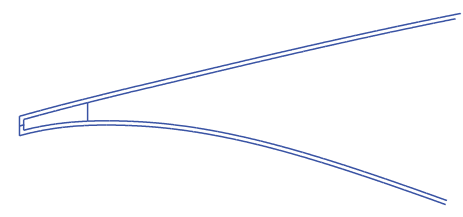

Figure 4.7. Internal structure of the airfoil showing the split in the Nose structure.

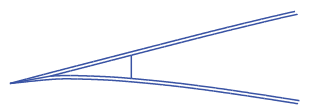

Figure 4.8. Internal structure of the airfoil showing the split in the Nose structure.

\subsubsection{Shear Web Design}

After generating these sectioned splines the shear webs were created as they complete the box beam structure by providing buckling stiffness. Joining the corresponding points a perfect shell structure defining the adaptive blades was created. The blade was thus divided into 2 major parts the upper region as well as lower region. The can be easily distinguished by focusing our attention on the line parting the 2 . The main aim of this modification in the blade structure internal layout was to provide more flexibility in designing of the blade keeping in mind the scenario of material adaptiveness.

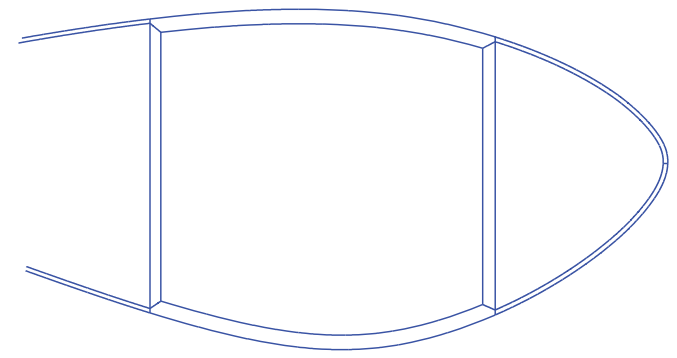

Figure 4.9. Internal structure of the airfoil showing shear web structure. 
Prior to this modification the blade was divided into 7 parts as explained earlier. After this modification the blade was divide into

- Nose upper

- Nose Lower

- Upper Spar cap

- Lower Spar cap

- Front shear web

- Rear Shear Web

- Tail upper part

- Tail lower part

- Trailing edge filler material

The old configuration used to deal with the nose on a whole thus there was just one lamination or manufacturing angle for the whole nose. This implied that if the fibers on the top part of the nose made an angle say of $20^{\circ}$ with the incoming wind flow then due to this configuration the lower part of the nose was compelled to make an angle of $-20^{\circ}$ with the wind flow. This severely compromised the blade in sense of stiffness and flexo torsional effects. Creating the blade in two parts gave us the flexibility to orient angles on each side of the blade such that they make the same angle with the wind flow. This orientation of the fibers generate an extra resistance to stall thus creating an optimum work condition. Similarly as the spar caps have also been separated from each other both of them can be given independent configurations which may constructively react to cater the requirements of a multi concept smart blade.

All the blades used for validations in the following chapter are based on this internal structure. The effects of this change were widely seen when compared to the same blade of earlier configuration. Also in various validation tests when the manufacturing angles were changed the effect of it on the blade behavior were seen prominently.

Seen below are the two kinds of true airfoils generated with the new airfoil structure generation code. 


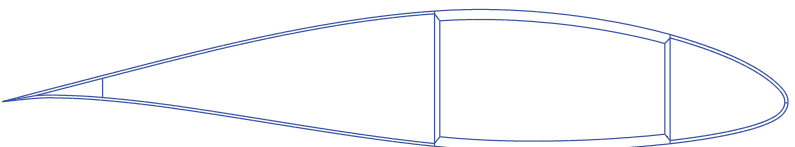

Figure 4.10. Pointed tail airfoil with new internal structure.

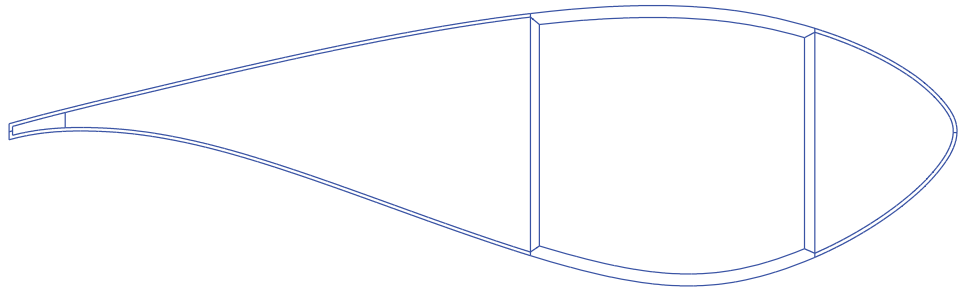

Figure 4.11. Blunt tail airfoil with new internal structure. 


\section{THE LEVEL-1 FLOW MODEL}

This chapter throws light on the theoretical basis of the LEVEL- 1 flow model which then further interacts with its structural counterpart. The level 1 flow model provides us with the aerodynamic loads which are sensitive enough to interpret and utilize all the complex deformation modes which are in turn provided by the structural model. That being stated, even the fluid model should be able to describe a feedback which is of the same details as that of the structural model. This helps one to accurately model the fluid-structure interaction on the rotor blades. The computation code that is being used to compute the fluid structure interaction is the one that is developed by the National Renewable Energy Laboratory (NREL), Aerodyn [32,33]. The base of our aerodynamic model just like the Aerodyn is the established Blade Element Momentum Theory. The structural model used by us provides a high level of detail. The generation of coherence between the structural code and the aerodynamic one required a considerable amount of reformation in the existing computational code. The reformation helped in overcoming numerous limitations that were presented by the existing code [34].

The chapter presented below begins with a brief introduction to the classic formulation of the BEM theory focusing its application to Horizontal Axis Wind Turbines (HAWT). The introduction Is then followed by the formulation designed by MTU's team lead by Dr. Ponta to overcome the limitations exhibited by the classic theory.

\subsection{Theoretical Background: The Classic Blade Element Momentum theory}

The BEM theory, usually attributed to Betz and Glauert[35], has its origin from two different theories viz. blade element theory and momentum theory. The blade element theory associates with lift and drag that are generated by the interaction of the airfoil shape and its interaction with the wind flow over it. The momentum theory on th e other hand deals with the conservation of linear as well as angular momentum on a control volume. This control volume is assumed to be and usually modeled as stream tube. This helps in 
the analysis of the force over the rotor blades [36].

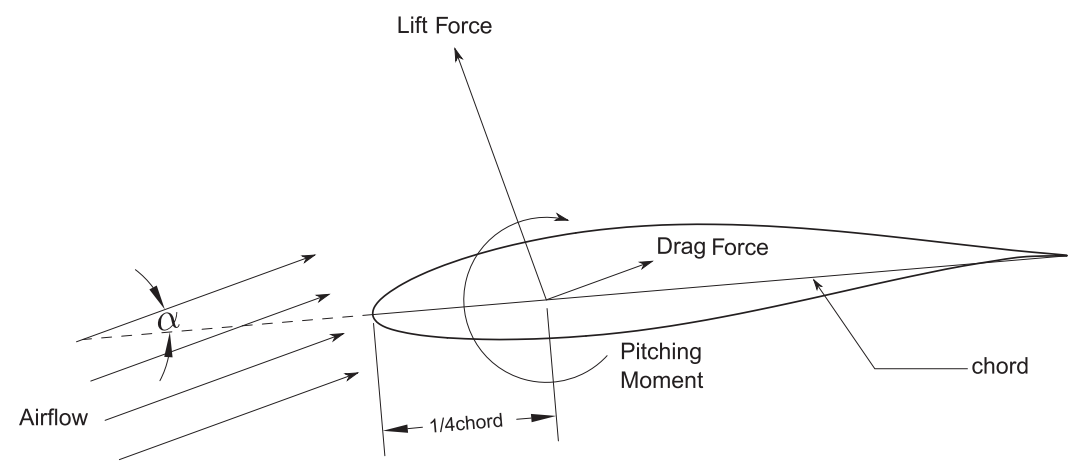

Figure 5.1. Aerodynamic efforts over the airfoil.

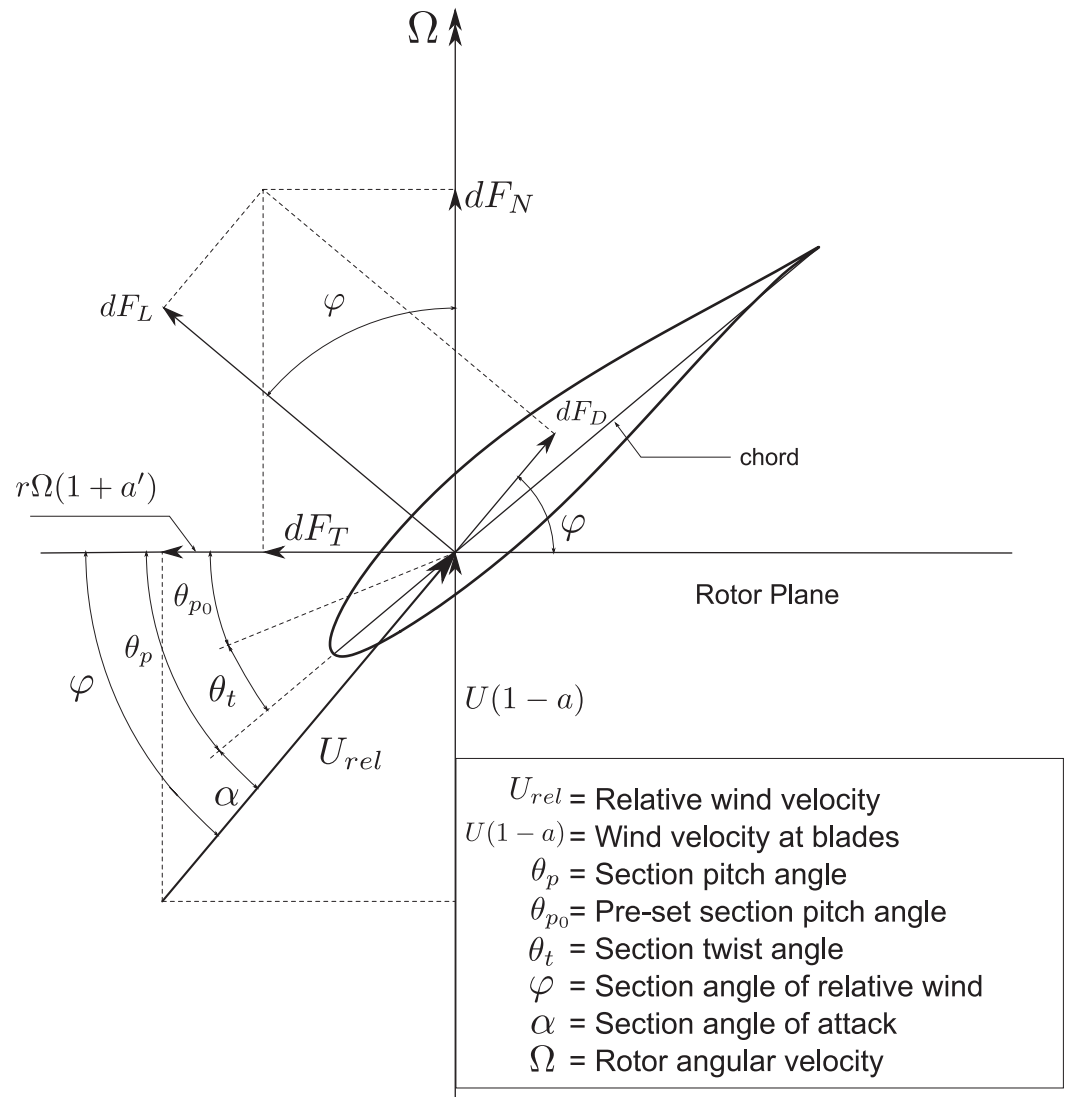

Figure 5.2. HAWT blade cross-sectional analysis. 
The Fig. 5.2 above briefly analyses the forces as well as the angles that are defined with respect to an airfoil subjected to an incoming airflow. With the working conditions of the blade as seen in the scenario above and with help of some basic understanding of aerodynamics the Urel can be described as

$$
U_{r e l}=\sqrt{(U(1-a))^{2}+\left(\Omega r\left(1+a^{\prime}\right)\right)^{2}}
$$

where $a^{\prime}$ is the angular induction factor which represents the increment on the relative tangential velocity in the blade's cross-section due to the rotation of the wake behind the rotor.

Consider a rotating blade of a HAWT. Now any small element on this blade as known as the blade element sweeps an annulus in its motion along the path [37] (see figure ??. The blade element momentum theory here deals with the force of this blade element with the momentum change of all the air passing through the described annular area by the same element. The radial flow interactions between any contiguous annuli are neglected. For the above assumption to be true the axial induction factor should not be a function of radial distance and thereby must be constant along the radius. Experimental demonstrations have coined this radial assumption to be acceptable. The BEM theory provides us with an iterative process for the computation of the induction factors $\left(a, a^{\prime}\right)$. These obtained induction factors are then used to obtain the aerodynamic forces acting on the rotor. The process of computation of the induction factor has to be iterative as the induction factors depends upon the angle of attack and the relative wind angle for each blade section which in turn is obtained from the lift and drag coefficients of the blade section in consideration.

An iterative process is used for the calculation of the induction factors begins with the definition of blade solidity and going further by finding the angle of relative wind. Once that is done the process is continued by incorporating the corrections due to the tip and hub losses. The blades aerodynamic coefficients are then calculated from the obtained angle of attack. Calculation of the local thrust thus enables us to obtain axial induction factor followed by the angular factor. The process is then repeated until convergence. Upon convergence $U_{r e l}$ is then calculated by the following equations

$i$ being the number of steps to convergence and with the local wind velocity given as:

$$
U_{r e l}(r)=\sqrt{\frac{U^{2}\left(1-a_{i}(r)\right)^{2}}{\sin ^{2}\left(\varphi_{i}(r)\right)}},
$$

These disturbed forces are termed as Linlenthal forces. They correspond to the coefficents of the same name. These forces refer to the airfoil chord and are different from the 
one shown in the fig 5.2 (capital subindex) which are in reference to the rotor plane.

$$
\begin{gathered}
d F_{N}(r)=d F_{l}(r) \cos \left(\alpha_{i}(r)\right)+d F_{d}(r) \sin \left(\alpha_{i}(r)\right) \\
d F_{T}(r)=-d F_{l}(r) \sin \left(\alpha_{i}(r)\right)+d F_{d}(r) \cos \left(\alpha_{i}(r)\right) .
\end{gathered}
$$

\subsubsection{Corrective factors to BEM theory}

BEM theory was originally designed for asymmetric flow. The wind turbines in general are operated at yaw angles relative to the incoming wind flow. This leads to a skewed wake formation behind the rotor. In order to account for this skewed wake effect the BEM model needs to be corrected appropriately $[38,39]$.

The structural model of Aerodyn [32] is modeled to take into consideration the blade aerodynamics with the wind turbine tower established. It implements the models developed by Bak et al. [40] and SRJ Powles [41] that provide the influence of the tower on the local velocity field at every point in the local neighborhood of the tower. The model is accountable for the increase in the speed of the wind flow around the sides of the tower and also updates the cross stream velocity component in the near tower flow field.

\subsubsection{Limitations of the classic BEM theory for large deformations}

The current wind turbine industry reflects the tendency to increase the size of these machine [12] drives not only to bigger blades but also to a more flexible and relatively lighter blade.High rotation speeds are observed in these new blades due to the large deformations that they exhibit. These deformations are a result either of the flexibility of these blades or the pre-conforming process. The pre-conforming process can be on either side which makes the deformation more of a complicated variable to deal with. These large deformation becomes a point of failure for the traditional BEM theory. The actual mathematical formulation implies the blade section to always remain perpendicular to a radially outward line which is co-planar to the actuator disk which in turn in coincident to the rotor plane. Hence even though the equations of all aerodynamic efforts and the change of momentum of all the stream tubes remains valid the mathematical formulation fails at representing the large section rotation. It is because of this misrepresentation in the effect of the aerodynamic loads a new mathematical formulation is required to transfer the velocities obtained from the momentum theory to the blade element's plane. The projected velocities are then further required to be reprojected onto the plane of the stream tube actuator disk. In all this

projection and reprojections the forces obtained by the original BEM theory with the help of ones obtained from the change of momentum in the plane of stream tube are still valid. 


\subsection{Large Sectional Rotation BEM (LSR-BEM)}

As discussed in the prior section a new mathematical formulation is required to overcome the computational errors occurring due to large deformations.

$$
\begin{aligned}
& \mathbf{R}_{\mathbf{x}(\theta)}=\left[\begin{array}{ccc}
1 & 0 & 0 \\
0 & \cos (\theta) & -\sin (\theta) \\
0 & \sin (\theta) & \cos (\theta)
\end{array}\right] \\
& \mathbf{R}_{\mathbf{y}(\theta)}=\left[\begin{array}{ccc}
\cos (\theta) & 0 & \sin (\theta) \\
0 & 1 & 0 \\
-\sin (\theta) & 0 & \cos (\theta)
\end{array}\right] . \\
& \mathbf{R}_{\mathbf{z}(\theta)}=\left[\begin{array}{ccc}
\cos (\theta) & -\sin (\theta) & 0 \\
\sin (\theta) & \cos (\theta) & 0 \\
0 & 0 & 1
\end{array}\right]
\end{aligned}
$$

The above matrices are the rotation matrices around the $x, y$, and $z$ co-ordinate axis. Dot product of these matrices in a certain order help us in carrying out many more complex rotations. The product of $\mathbf{R}_{\mathbf{z y x}}=\mathbf{R}_{\mathbf{z}(\gamma)} \mathbf{R}_{\mathbf{y}(\beta)} \mathbf{R}_{\mathbf{x}(\alpha)}$ will produce $\mathbf{R}_{\mathbf{z y x}}$ matrix. Pre multiplication of any vector $\mathbf{v}$ will give a $\mathbf{v}^{\prime}=\mathbf{R}_{\mathbf{z y x}} \mathbf{v}$ which is caused by the rotation of the original vector $\mathbf{v}$ by $\alpha$ radians in the $x$ axis, $\beta$ in the $y$ axis, and $\gamma$ radians in the $z$ axis. The transpose of $\mathbf{R}_{\mathbf{z y x}}$ matrix will however not rotate the vector $\mathbf{v}^{\prime}$ back into the original co-ordinate system. More details in [42-44].

- $\mathbf{R}_{\mathbf{x}, \theta}^{T}=\mathbf{R}_{\mathbf{x}, \theta}{ }^{-1}$

- $\operatorname{det}\left(\mathbf{R}_{\mathbf{x}, \theta}\right)=1$

- $\mathbf{R}_{\mathbf{x},(\theta+\mathbf{r})}=\mathbf{R}_{\mathbf{x}, \theta} \cdot \mathbf{R}_{\mathbf{x}, \mathbf{r}}$

- $\mathbf{R}_{\mathbf{x}, \mathbf{0}}=\mathbf{I}$ (where $\mathbf{I} \in \mathbb{R}^{n}$ is the identity matrix).

- The eigenvalues of $\mathbf{R}_{\mathbf{x}, \theta}$ are: $\left\{1, e^{ \pm i \theta}\right\}=\{1, \cos (\theta)+i \sin (\theta), \cos (\theta)-i \sin (\theta)\}$.

Above mentioned are the properties for any rotation matrix $\mathbf{R}_{\mathbf{x}, \theta} \in \mathbb{R}^{3}$ where $x$ is a rotation axis and $\theta$ a rotation angle $[42,43]$.

The wind velocity vector Uh which faces the differential annulus of the actuator disk is then defined. The components of this vector are affected by the induction factors, both the 
axial factor $a$ as well as the tangential factor $a^{\prime}$. The $h$ which appears in the subscript in the nomenclature of this vector signifies the definition of this vector in the $h u b$ co-ordinate system ((see figure 5.3) according to standards from the International Electrotechnical Commission (IEC) [45].)

$$
\mathbf{U h}=\left[\begin{array}{l}
U w h_{1}(1-a) \\
U w h_{2}+\Omega r_{h}\left(1+a^{\prime}\right) \\
U w h_{3}
\end{array}\right],
$$

We define $U w h$ as the velocity of the incoming wind projected on to the hub $h$ co-ordinate system, with $\Omega$ being the angular velocity of the rotor and $r_{h}$ being the radial distance of the airfoil section in the $\mathrm{h}$ co-ordinate system.

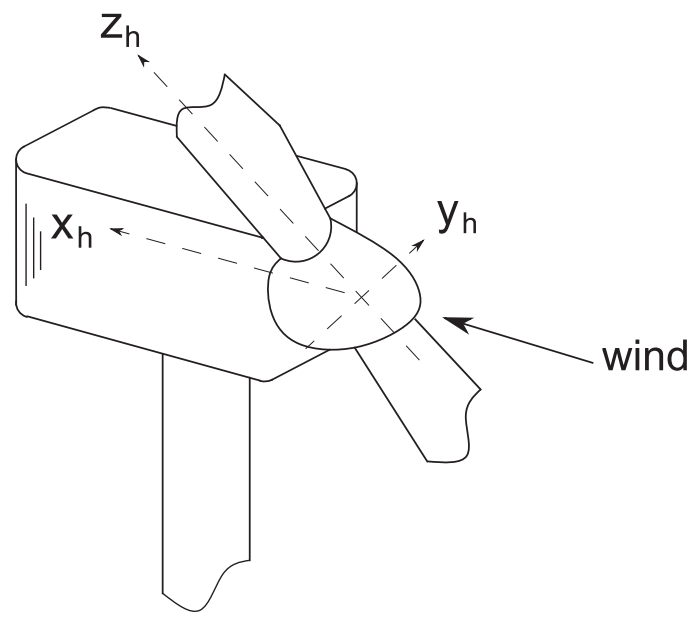

Figure 5.3. Hub coordinate system (facts in the figure according to standards from the International Electrotechnical Commission (IEC) [45])

To calculate the relative velocity that affects the blade element we go ahead projecting Uh through the hub coordinate system till reaching the blade section's coordinate system. The following matrices help in the ultimate transformation from the h coordinate system to the blade section's coordinate system.

The coning rotation matrix, a linear operator helps us with a basic rotation about the $y$ axis in the hub coordinate system where in $y_{h}$ (see figure 5.3), where $\theta_{c n}$ is the conning angle for the rotor.

$$
\mathbf{C}_{\theta_{\mathbf{c n}}}=\left[\begin{array}{ccc}
\cos \left(\theta_{c n}\right) & 0 & \sin \left(\theta_{c n}\right) \\
0 & 1 & 0 \\
-\sin \left(\theta_{c n}\right) & 0 & \cos \left(\theta_{c n}\right)
\end{array}\right]
$$


After successfully orienting the wind velocity for the coning effects on the rotor it is then oriented into an existing pitch angle defined for the blade. This pitch angle $\theta_{p}$ is the addition of the instantaneous pitch control angle and the pre-set pitch angle $\theta_{p_{0}}$ which may be defined earlier.

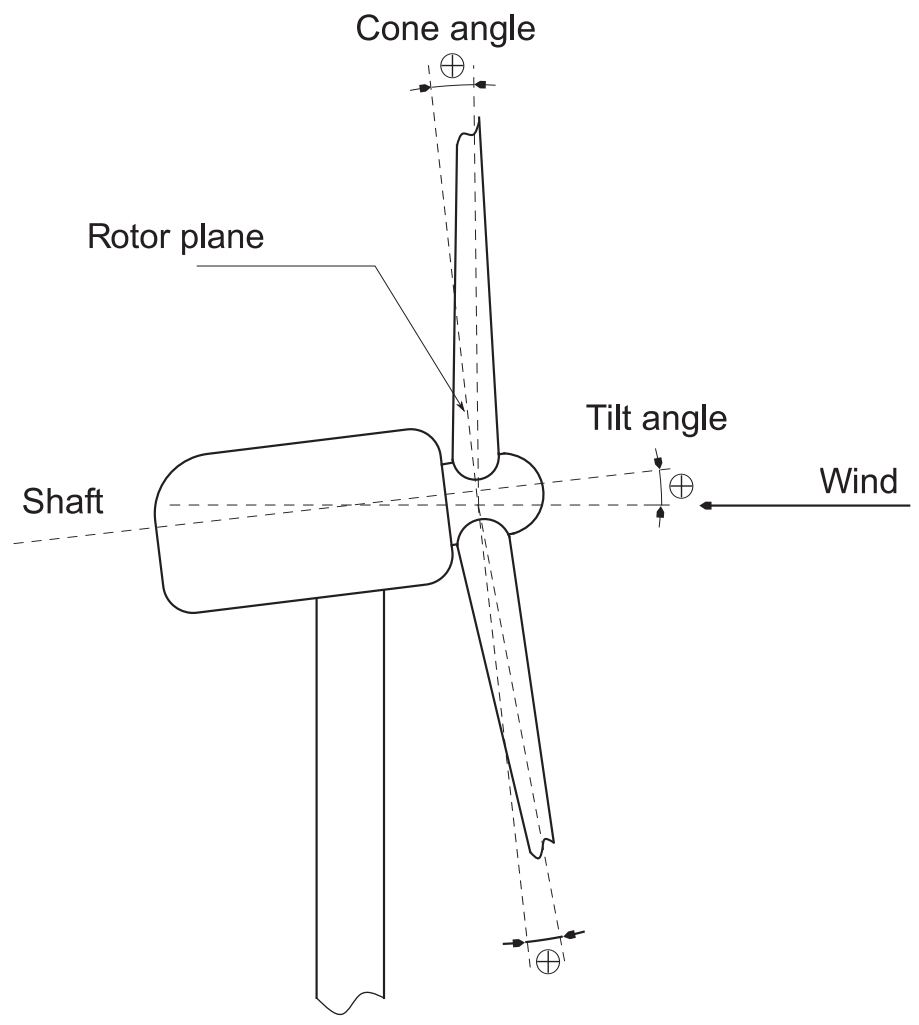

Figure 5.4. Tilt and cone angles for upwind wind turbines, (facts in the figure as per the standards from the International Electrotechnical Commission (IEC) [45])

$\theta_{p_{c}}$ comes from the external control actions. The pitching rotation matrix $\mathbf{C}_{\theta_{\mathbf{p}}}$ defines a rotation about the $z$ axis resulting into the transformation of the coning matrix into blade coordinate system (see figure 5.5) referred to by the $b$ subscript

$$
\mathbf{C}_{\theta_{\mathbf{p}}}=\left[\begin{array}{ccc}
\cos \left(\theta_{p}\right) & -\sin \left(\theta_{p}\right) & 0 \\
\sin \left(\theta_{p}\right) & \cos \left(\theta_{p}\right) & 0 \\
0 & 0 & 1
\end{array}\right],
$$

where $\theta_{p}=\theta_{p_{0}}+\theta_{p_{c}}$. Thus, any transformation from $h$ to $b$ would be given by: $\mathbf{C}_{\mathbf{b h}}=$ $\mathrm{C}_{\theta_{\mathbf{p}}} \mathrm{C}_{\theta_{\mathbf{c n}}}$.

Two more transformations are required for further conversion of the wind velocity from the blade's coordinate system to the instantaneous co-ordinate system denoted by $r$ 3.1. As 


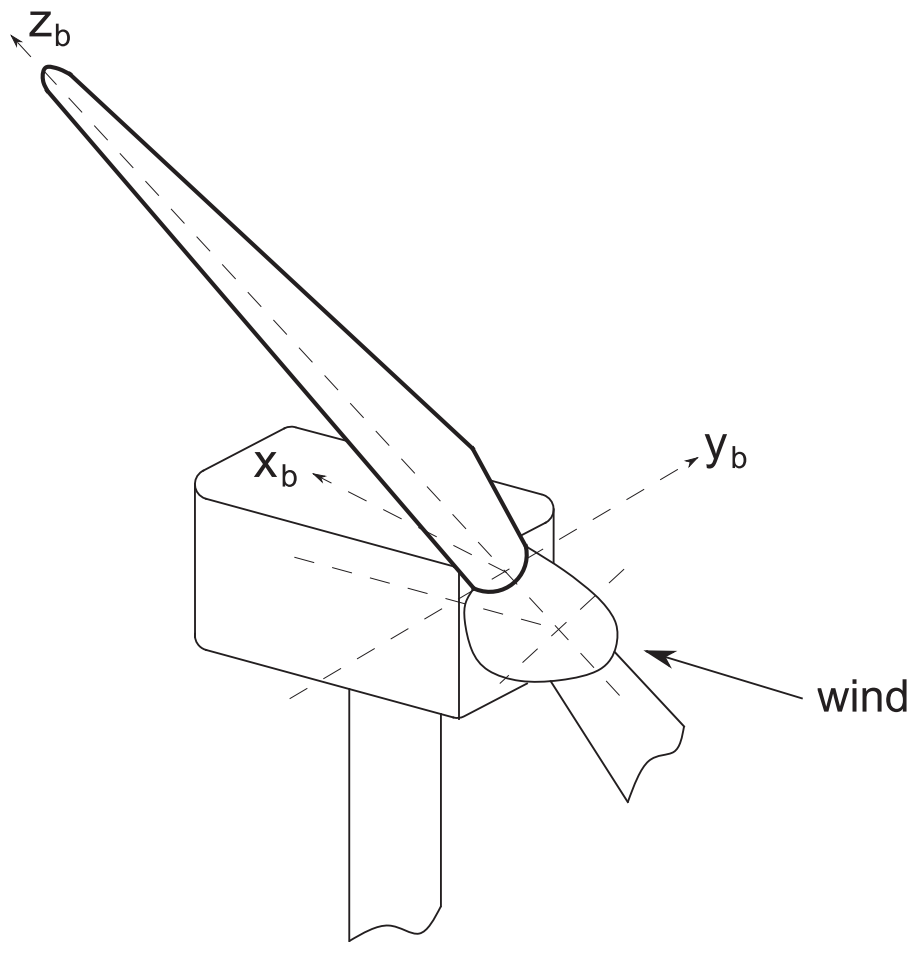

Figure 5.5. Blade coordinate system (facts in the figure according to International Electrotechnical Commission Standards (IEC) [45])

implied as it can be we need two more matrices to carry out the transformation. One out of these two matrices contains the information of the blade section geometry from the time when the blade was designed and manufactured. The blade as we discussed earlier might have pre-defined curvatures along the longitudinal axis. This curvature can either account for an initial twist in the blade geometry along the longitudinal axis or may also define a twist along with a pre conicity at the rotor on the rest two axes(i.e. conning/sweeping). At the blade design stage itself a set of transformation matrices containing the information of the orientation of the blade in three dimensional space is already defined. These matrices store this information for each position along the longitudinal axis [46]. To this end, we compute the Fernet-Serret formulas to define the curvature along the now curvilinear longitudinal blade axis. These newly defined formulae now help in establishing the tangent, normal, and binormal unit vectors for the given curve. The Fernet-Serret coordinate system is also termed as the $T N B$ coordinate system because of the three unit vectors that it helps in defining $[46,47]$. In context to the particular twist specified in the blades aerodynamic design further rotation of each blade section around the tangential axis of the TNB coordinate system is needed. With the combination of all these rotations we can obtain specific transformations of each blade section in particular along the span. This matrix is known as the $\mathbf{C}_{\mathbf{R b}}$ matrix as it helps in transformation from the global coordinate system of the 
blade $\mathrm{b}$ into the coordinate system of the blade sections in its undeformed state $R$, as seen in section 3.1 .

The last transformation is obtained by $\mathbf{C}_{\mathbf{R b}}$ matrix which carries out the transformation from the undeformed blade section coordinate system into the instantaneous blade section coordinate system. This matrix contains the information to transform all the vectors from $R$ to $r$ coordinate system after the occurrence of deformation. Being one of the key variables in syncing the information between structural and aerodynamic model the $\mathrm{C}_{\mathrm{rR}}$ matrix has to be updated at each time step in dynamic simulations.

Once all the require transformations are done we obtain Uh vector in the instantaneous blades deformed coordinate system $r$.

$$
\mathrm{U}_{\mathrm{rel}}=\left(\mathrm{C}_{\mathbf{r R}} \mathbf{C}_{\mathbf{R b}} \mathbf{C}_{\theta_{\mathbf{p}}} \mathbf{C}_{\theta_{\mathrm{cn}}} \mathrm{Uh}\right)+\mathbf{v}_{\mathrm{str}}
$$

$\mathbf{v}_{\text {str }}$ is the blade section structural deformation velocity. It comes from the structural model. The magnitude of $\mathbf{U}_{\text {rel }}$ and the angle of attack $\alpha$ are used to calculate the aerodynamic forces on the airfoil section under consideration through already obtained lift and drag coefficients.

$$
d F_{l}=\frac{1}{2} \rho C_{l}\left|U_{r e l}\right|^{2} c, \quad d F_{d}=\frac{1}{2} \rho C_{d}\left|U_{r e l}\right|^{2} c
$$

The aerodynamic loads acting on the blade element can be represented with the help of the following matrix.

$$
\mathbf{d F r}=\left[\begin{array}{c}
0 \\
d F_{d} \\
d F_{l}
\end{array}\right]
$$

The obtained loads are then projected on to the $h$ coordinate system via

$$
\mathbf{d F h}=\mathbf{C}_{\theta_{\mathbf{c n}}}{ }^{T} \mathbf{C}_{\theta_{\mathbf{p}}}{ }^{T} \mathbf{C}_{\mathbf{R b}}{ }^{T} \mathbf{C}_{\mathbf{r R}}{ }^{T} \mathbf{C}_{\text {Lthal }} \mathrm{dFr},
$$

$\mathrm{C}_{\mathrm{Lthal}}$ matrix projects the lift and drag forces in the chord wise and chord normal directions in the $r$ coordinate system.

$$
\mathbf{C}_{\text {Lthal }}=\left[\begin{array}{ccc}
1 & 0 & 0 \\
0 & -\cos (\alpha) & \sin (\alpha) \\
0 & \sin (\alpha) & \cos (\alpha)
\end{array}\right]
$$


In the end as described in the classic BEM theory the force is equated to the rate of change of momentum in the annular stream tube described by the blade element section under consideration. The normal component of the force $\left(d F_{N}\right)$ to the rotor disk is equated against the axial rate of change of momentum while the tangential component $\left(d F_{T}\right)$ is conveniently equated to the change in angular momentum.

The equation of the axial force can be written as

$$
\begin{gathered}
\mathbf{d F h}=\left[\begin{array}{c}
d F_{N} \\
d F_{T} \\
0
\end{array}\right] \\
B d F_{N}=4 \pi \rho|\mathbf{U h}|^{2} a(1-a) r .
\end{gathered}
$$

With the additional axial force on the annulus caused by the increase in dynamic head as a result of a drop in wake pressure due to the rotation of the wake. This extra term was proposed by Burton et al [37].

$$
\frac{1}{2} \rho\left(2 a^{\prime} \Omega r\right)^{2}
$$

Similarly the tangential force will be given by

$$
2 \pi r \frac{1}{2} \rho\left(2 a^{\prime} \Omega r\right)^{2}
$$

Taking 5.16 into account, equation5.14 becomes:

$$
\begin{gathered}
B d F_{N}=4 \pi \rho F_{f}\left(|\mathbf{U h}|^{2} a(1-a)+\left(2 a^{\prime} \Omega r\right)^{2}\right) r \\
B d F_{T}=4 \pi \rho F_{f}|\mathbf{U h}|(\Omega r) a^{\prime}(1-a) r^{2},
\end{gathered}
$$

the explicit expression for the tangential induction factor is thus:

$$
a^{\prime}=\frac{-B d F_{T}}{4 \pi \rho F_{f}|\mathbf{U h}| \Omega(1-a) r^{2}}
$$




\subsubsection{Iterative solution of the induction factors}

Iterative approach is used to obtain the value of the induction factors at each time step of the devised aeroelastic solution. Minimizing the residual equation 3.32 we obtain the axial induction factor. The fzero function of MATLAB is used for the minimization of the residual equation. fzero function uses bisection, inverse quadratic and secant interpolation methods to find the root of a continuous function with one variable. MATLAB always monitors for error checks and convergence criteria for such closed loop methods, the reason these methods are used for reduction $[48,49]$.

On complete convergence of the interference factors a final correction still remains to be done on the axial induction factor $a$ as when the incoming wind flow is not exactly aligned perpendicular to the actuator disk. Corrections to this skewed wake are found in the Method of Acceleration Potential developed by Prandtl. The new axial induction factor after incorporating the corrections provided Pitt and Peters [38,39] relying on the yaw angle instead of the wake skew angle is as follows :

$$
a=a_{0}\left(1+\frac{15 \pi}{32} r \tan \left(\frac{\psi}{2}\right) \sin (\Omega t)\right),
$$

$a_{0}$ here is the previously converged axial induction factor with $\psi$ being the yaw angle of the rotor and $\Omega t$ being the instantaneous angular velocity of the rotor. On the successful convergence of the iterative algorithm the aerodynamic force are then calculated on each of the blade sections using the equations 5.5 to 5.13 and method discussed earlier. The pitching moment is also calculated using the classic pitch coefficient $\mathrm{Cm}$.

\subsubsection{Interference model for the "turbulent-wake" state}

$C T=4 a(1-a)$. With this quadratic equation obtained from the classic beam theory with CT being the coefficient of thrust the CT obtains its maxima at $a=0.5[36]$. Beyond this point 5.1 the windturbine exits from the state termed as the wind-mill state and enters into the furbulent wake state: In this state the basic assumptions of the classical BEM theory become void. A correction is now required for the assumptions to stand ground and to take care of the part of wind which flows upstream at the far wake region. This reversal of flow is not physically possible and it doesn't take place. What really happens is that more wind enters the flow thereby increasing the wake and the turbulence. As a result of all this addition to the flow the rotor slows down but the thrust keeps on increasing[36,37].

Glauert overcame this limitation of the BEM by fitting a parabolic function to the numerical data obtained from the experiments of Lock et al[50] on the wind turbines operating 


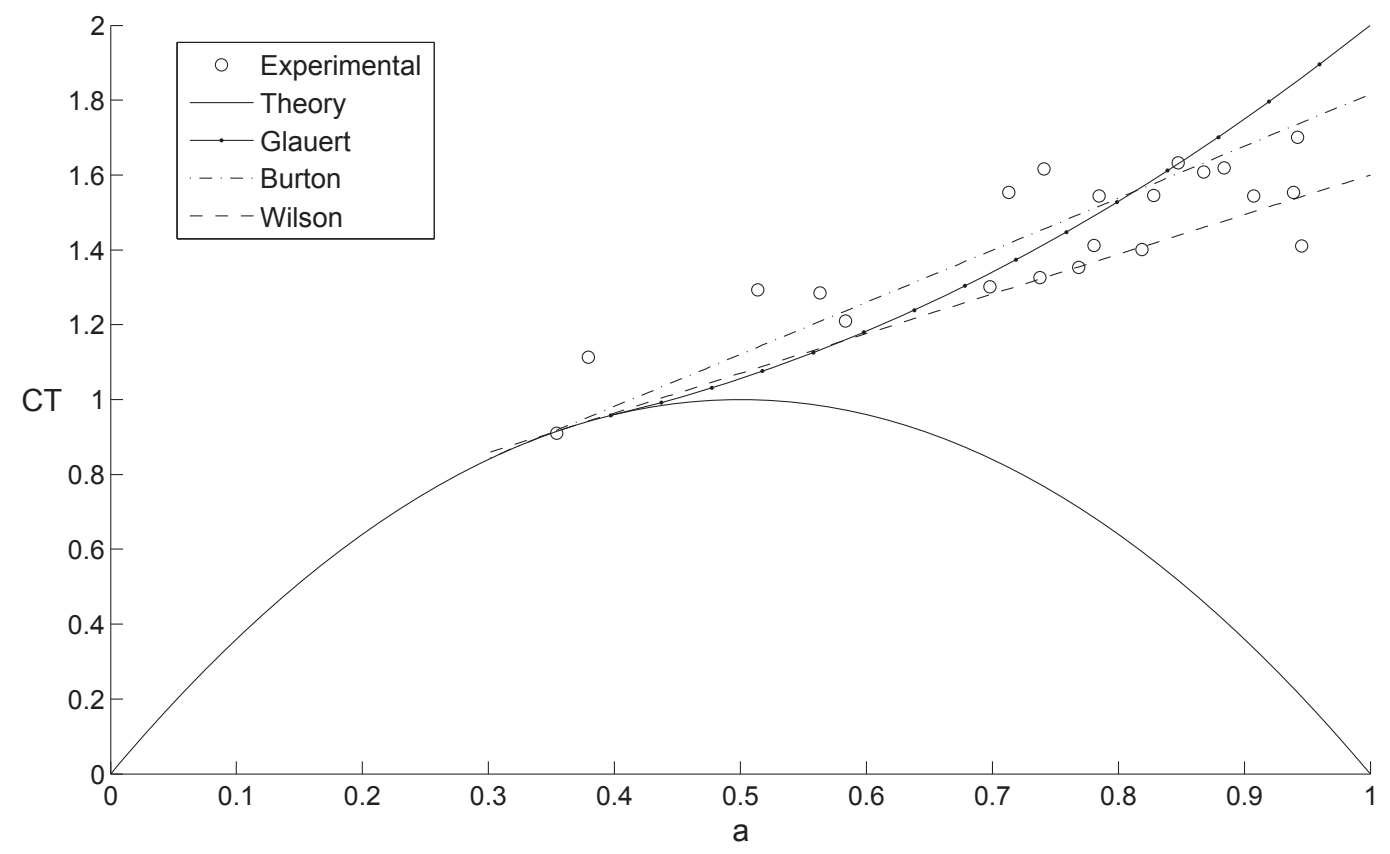

Figure 5.6. Fitting the Lock experimental data [52] for wind turbines operating in the turbulent wake state.

in turbulent state. Glauert's function is a tangent to the $C T$ curve at $a=0.4$. Other authors as seen from the figure also proposed various fitting functions based on the experimental data on similar kind of wind turbines. However when the corrections to the tip and the hub losses are considered there can be seen a critical discontinuity between the $C T$ curve and the fitting function. Marshall Buhl,Jr's [51] empirical relationship for the axial induction factor and the thrust coefficient for turbines in a turbulent wake state not only solves the discontinuity issue but also takes into account the tip and the hub losses [51].

Buhl's empirical relation however is unable to minimize the error of the existing experimental data. A new empirical relationship based upon fitting a power-law function to Lock's experiment is used

$$
\Psi(a)=1.724(a-0.280)^{0.225}
$$

This power law function starts to depart from its theoretical parabolic shape for induction factors more than 0.3 as observed by Locks experiments. Figure 5.7 shows the Glauert's, Buhl's and the proposed power-law fitting function for the $C T$ curve affected by the loss factor $\left(F_{t}\right)$ of 0.9 . Buhl's empirical relation as seen overcomes the problem of discontinuity [51]. Our devised power law also overcomes the discontinuity problem by 
directly cutting the $C T$ curve rather than being a tangent to it. Intersection of both these curves is taken into consideration and then on basis of the region a suitable regime for the computation of the actual induction factor is chosen. Table 5.1 shows the mean square root error MSE for various iterative approaches in calculation of $C T$. The power here clearly produces the error one magnitude lower to the errors of other iterative methods.

Table 5.1

Mean Square Error of the different empirical approximations with Lock's experimental data.

\begin{tabular}{lc}
\hline Empirical relation & $M S E=\frac{1}{n} \sum_{i=1}^{n}\left(x_{i}-\overline{x_{i}}\right)^{2}$ \\
\hline Glauert & 0.0291 \\
Buhl & 0.0291 \\
Wilson & 0.0201 \\
Burton & 0.0166 \\
Power-law & 0.0094 \\
\hline
\end{tabular}

Thus if at certain section during the computation of the interference model the axial induction factor comes out to be greater than equal to the value obtain upon the intersection of the $C T$ curve with the power-law the minimization of the residual is carried out for the following equation instead of equation 5.17.

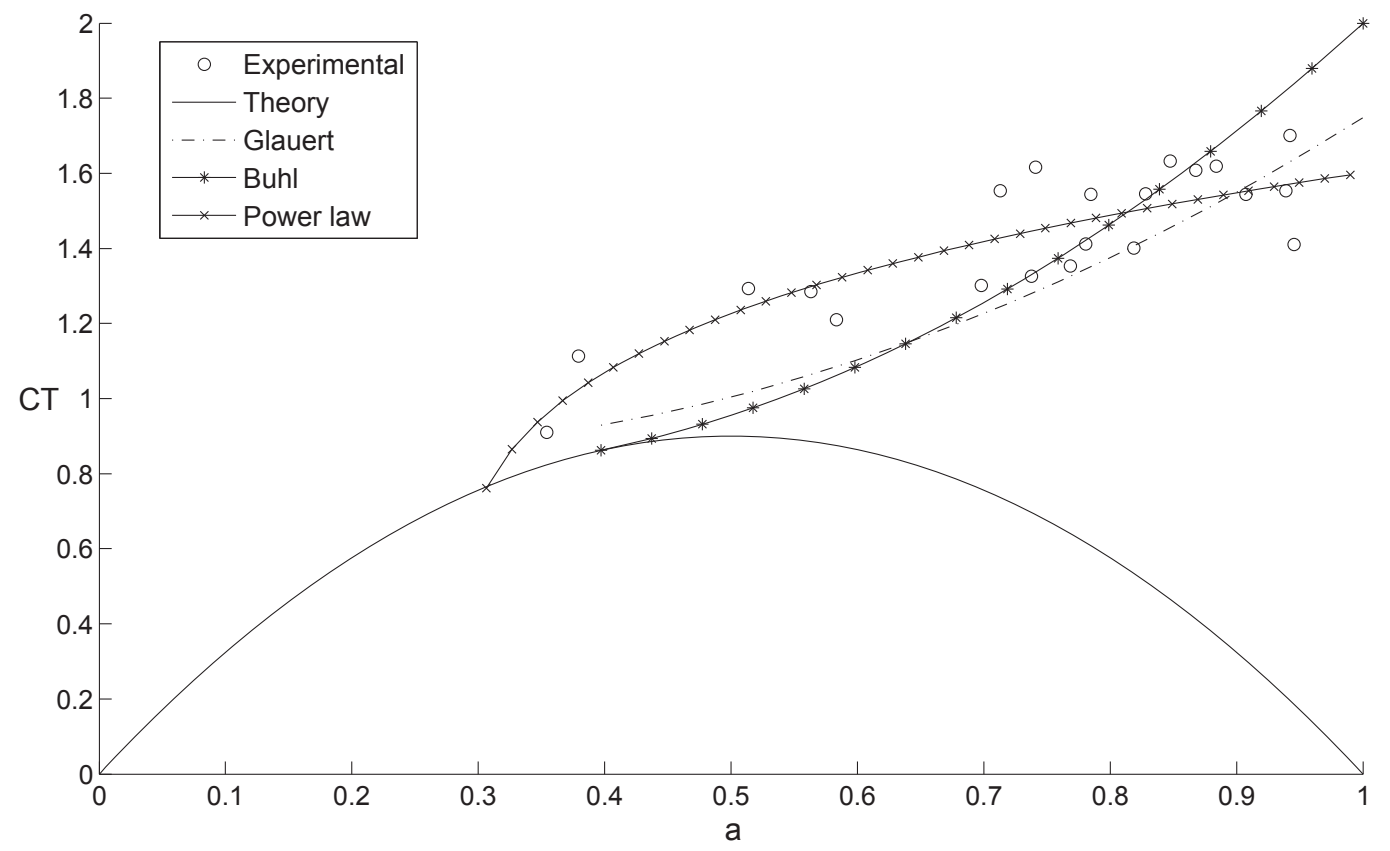

Figure 5.7. Power Law, Buhl's, and Glauert's empirical relations fitting the Lock experimental data [52]. 


$$
0=\Psi(a)-C T_{l}
$$

The local thrust coefficient $C T_{l}$ is given by:

$$
C T_{l}=\frac{B d F_{N}}{\rho\left|U_{(h)}\right|^{2} \pi r} .
$$

\subsection{The Flow Model Interface (FMI)}

As described earlier 2.2 the FMI is a module that computes the aerodynamic forces which are further to be used in the structural model. This section helps in understanding various steps required to be carried out prior to the solving of the LSR-BEM interference model.

\subsubsection{Pre-processing of the airfoil aerodynamic coefficients}

The aerodynamic coefficients for a given airfoil are obtained through the static airfoil data for provided by Aerodyn [32,33] as a result of a series of wind tunnel tests. The data processing in FMI however helps in overcoming various limitations of Aerodyn[34]. The static data obtained is required for the preprocessing of the data on an external program AirfoilPrep [33,53]. This is used for the correction of the aerodynamic coefficients in oreder to take into account the three-dimensional effects like rotational augmentation $[54,55]$. The correction is performed before initiating the solution to the aero-elastic problem as it is not updated during the time integration procedure. The second is for the implementation of dynamic stall however that is out of the scope of this thesis.

FMI allows the addition of multiple data tables for different airfoils. This data can pulled accordingly as per the instantaneous aerodynamic situations on the rotor and also in case of modified airfoils with active control surfaces. The FMI does process the aerodynamic coefficient tables and provides us with the updated variables to the Stall-Delay model from Du \& Selig [54] to include the rotational augmentation effects to the coefficients of lift in real time. Corrections for the drag coefficients are also obtained similarly from Eggers' model [55] and are computed for each time step in the simulation. The correction of the aerodynamic coefficients is followed by the Viterna Extrapolation [56] is applied to, by FMI as this assures the availability of the data for a range of anlge of attacks ranging from $\pm 180^{\circ}$. Its implementation is same as that in AirfoilPrep [33]. The significant difference between FMI and this are the rotational effects. Viterna extrapolates the lift and drag coef-

ficients assuming that the airfoil behaves as a flat plate for higher values of angle of attack 
[56] rather than considering them as airfoils.

airfoil coefficients for two airfoils, a thick airfoil from the DU family, the DU 91-W250, and a thin airfoil NACA 64-618. [Lucas]

\subsubsection{Coupling with the structural model}

LSR-BEM has the ability to incorporate all the deformations computed by the structural model at each time step. As opposed to, in classical BEM theory where the blade sections are constantly positioned in the radial direction; in LSR-BEM these blade sections are no longer at a constant radial positioning. These blade sections not only reflect the radial displacements but also define the displacements in other two directions. Thus they completely define themselves in space. The exact spatial positons of these blade sections in the hub coordinate system is calculated with the help of these projection matrices

A major limitation of Aerodyn [32,33] is overcome by infusing the structural results to the computation of the interference model at each time step. FAST [57], the velocity of blade sections due to deformation is received upon by Aerodyn through the structural models. This velocity is then added to the incident velocity of the wind at the blade sections under consideration. Aerodyn however computes the induction factors along with the corresponding aerodynamic loads by assuming the velocities relative to the undeformed configuration [34] as Aerodyn was formulated taking into mind only small deflections. Aerodyn-FAST also cannot deal with coupled deformations such as flexo-torsional or flexoflexural cause of the limitations imposed by the structural theory used.

\subsubsection{Wind input data}

The LSR-BEM interference model is always provided with the updated information of the rotor incoming wind for all aerodynamic load calculation by the Wind input data in FMI. For compatibility purposes with the existing databases the model in work is similar to the one used in Aerodyn [32]. It can also be called as a point wind file as the calculation of the wind is done merely by computing the shear applied to that very point where the wind is to be calculated. The model also doesn't consider any turbulent velocity components. A pre set file containing the data history as shown below is used to define the wind input file 
- Time

- Horizontal wind speed $\quad U_{\infty}$

- Instantaneous wind direction $\delta$

- Vertical wind speed $\quad U_{z}$

- Horizontal linear shear $\quad S h_{h l}$

- Vertical power-law shear $\quad S h_{v p}$

- Vertical linear shear $\quad S h_{v l}$

- Gust (horizontal) velocity $\quad U_{g s t}$

Unlike in Aerodyn the time evaluation of these parameters is done by interpolation of a piecewise cubic Hermit interpolating polynomials. Compared to a linear interpolation the higher ordered cubic interpolation provides us with a smoother interpolation and also takes into consideration all the available data points.Reorientation of the blade section position $\mathbf{p}_{\mathbf{G}}$ is required to take the yaw effects into consideration.

After the application of the rotor tower effects to Uw the FMI is used to reproject the same back into the hub coordinate system $h$.

$$
\mathbf{U h}=\mathbf{C}_{\Omega} \mathbf{C}_{\tau} \mathbf{C}_{\psi} \mathbf{U w t}
$$

Uwt is the wind velocity affected by the tower influence. The components of Uh are later modified by the interference factors $a$ and $a$. 


\section{NUMERICAL EXPERIMENTATION}

We use a set of rotor blades based on the 5-MW Reference Wind Turbine ( $R W T$ ) project developed by NREL [12] to serve as a datum for the validation of the model and also to test the various asspects of the blade aeroelastic concepts. The NREL's RWT that was based on RE power's 5MW wind turbine unlike its predecessor was designed for both onshore as well off shore installations. This model thus became a well representation of a state of the art, utility scale, multi megawatt commercial wind turbines.Even though the full specifications of the RE power model wasn't available to generate the exact design form it a baseline form the prototype blade was released byLM Glasfiber in 2001 for the Dutch Offshore Wind Energy Converter (DOWEC) $6 M W$ wind turbine project [58,59] it was later re adapted by NREL. These reports are considered to be the most accurate and most detailed sources of information that is available for large wind turbines. In addition to this, the NREL 5MW RWT project is also adopted as a model of reference by the International Energy Agency (IEA) European Union Upwind research program [60] and the Wind Annex XXIII Subtask 2 Offshore Code Comparison Collaboration(OC3) [61-63].

This chapter begins with a brief introduction to the structural feature of the blade and also the genereal aerodynamic properties. Let us first look at the basic building blocks of a blade, a good understanding of this will provide us with a sound vision of the level of details required in the computational codes to perfectly define a blade structurally. After this we will also review the blade internal structure, the finite element mesh associated with the structural computations. A series of tests are also carried out to for validation purposes to compare how closely the structural response of the blade has been replicated. Once the results are established, we go further by analyzing the aspects of adaptiveness of the blade for the original blade and then expand it to a set of newly designed configurations. The later more specific tests will tap the potential of Generalized Timoshenko model, already described in section 3.2. GTBM facilitates monitoring of the bending-twisting modes in the fully populated $6 \times 6$ stiffness matrix for the blade section. This matrix as discussed earlier is the stiffness matrix for the1-D beam problem which is solved for each timestep for the aeroelastic simulations. This in turn provides us with the edge of simulating the dynamic performance of the advanced adaptive blade. The computation also has the capacity to help in monitoring and studying the fluid structure interaction phenomena and also helps 
in design of experiments with blade as well as rotor properties as variables.

For the purpose of consistency with respect to all the different disciplines involved in this chapter we adopt a convention for the coordinate system used in order to describe the complex 3D blade structure. In this co-ordinate system the $\mathrm{x}$ axis is aligned to the span of the blade an all the variables related to the blade as a structural piece are denoted by a subscript $r$ unlike the nomenclature and definition of the International Electrochemical Commission [45] where the $z$ axis is the one aligned with the span and all the variables describing the blade as a structural component are denoted by the subscript $h$

\subsection{Basic blade modelling and aerodynamic properties}

As for the NREL's RWT project [12], the length of our rotor blade is also set to be $61.5 \mathrm{~m}$. All the basic aerodynamic properties such as twist angles, blade section chords as well as the distribution of the stations along the span corresponds to the data in [12]. These aerodynamic properties as well as the blade section present at each station is presented in a tabulated format in table 6.1. The figure 6.2 just complements the data in table 6.1

Table 6.2 shows the properties of Alternative Stall Blade designed by DU. This stall blade was used as a benchmark by Nimish Deshpande to develop a variable speed stall controlled wind turbine blade.

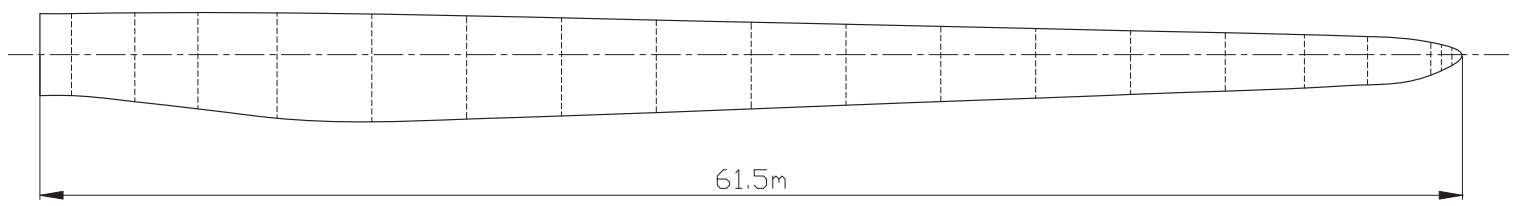

Figure 6.1. Distribution of chord along the blade.

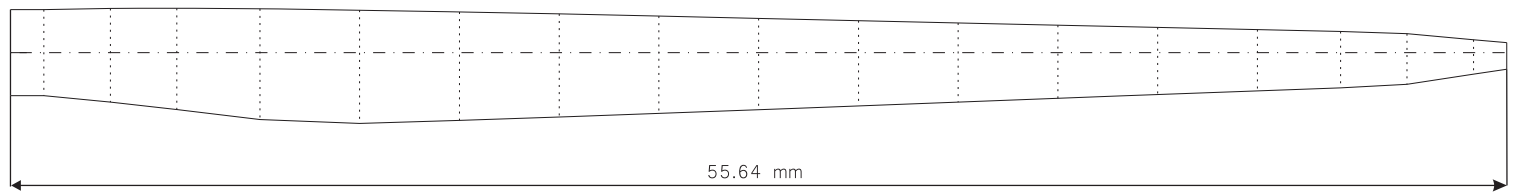

Figure 6.2. Distribution of chord along the stall blade.

The airfoil types are also matched to that of the original blade. Stations 3 and 4 in the table 6.1 can be termed as transitional airfoils. These sections have the shapes that are transforming themselves smoothly form that of a cylindrical one [12] at stations 1 and 2 
Table 6.1

Distributed blade aerodynamic properties.

\begin{tabular}{ccccl}
\hline Station & Location $[\mathrm{m}]$ & Twist angle $\left[{ }^{\circ}\right]$ & Chord length $[\mathrm{m}]$ & Airfoil type \\
\hline 1 & 0 & 13.3080 & 3.3900 & Cylinder \\
2 & 1.3653 & 13.3080 & 3.5380 & Cylinder \\
3 & 4.1020 & 13.3080 & 3.8588 & Ellipsoid-1 \\
4 & 6.8327 & 13.3080 & 4.1689 & Ellipsoid-2 \\
5 & 10.2520 & 13.3080 & 4.5256 & DU 00-W-401 \\
6 & 14.3480 & 11.4800 & 4.5811 & DU 00-W-350 \\
7 & 18.4500 & 10.1620 & 4.4704 & DU 00-W-350 \\
8 & 22.5521 & 9.0110 & 4.2193 & DU 97-W-300 \\
9 & 26.6480 & 7.7950 & 3.9771 & DU 91-W-250 \\
10 & 30.7500 & 6.5440 & 3.7370 & DU 91-W-250 \\
11 & 34.8520 & 5.3610 & 3.4906 & DU 93-W-210 \\
12 & 38.9479 & 4.1880 & 3.2451 & DU 93-W-210 \\
13 & 43.0500 & 3.1250 & 2.9993 & NACA 64-618 \\
14 & 47.1521 & 2.3190 & 2.7531 & NACA 64-618 \\
15 & 51.2480 & 1.5260 & 2.5350 & NACA 64-618 \\
16 & 54.6673 & 0.8630 & 2.3409 & NACA 64-618 \\
17 & 57.3980 & 0.3700 & 2.0325 & NACA 64-618 \\
18 & 60.1347 & 0.1060 & 1.4190 & NACA 64-618 \\
19 & 61.5000 & 0.0000 & 1.0900 & NACA 64-618 \\
\hline
\end{tabular}


Table 6.2

Distributed blade aerodynamic properties of Alternative Stall Blade designed by DU.

\begin{tabular}{ccccl}
\hline Station & Location $[\mathrm{m}]$ & Twist angle $\left[^{\circ}\right]$ & Chord length $[\mathrm{m}]$ & Airfoil type \\
\hline 1 & 0 & 11.6000 & 3.2045 & Cylinder \\
2 & 1.2352 & 11.6000 & 3.2045 & Cylinder \\
3 & 3.7112 & 11.6000 & 3.4868 & Ellipsoid-1 \\
4 & 6.1816 & 11.6000 & 3.7699 & Ellipsoid-2 \\
5 & 9.2752 & 9.9713 & 4.1228 & DU 00-W-401 \\
6 & 12.9808 & 7.9377 & 4.2087 & DU 00-W-350 \\
7 & 16.6920 & 6.8254 & 4.0332 & DU 00-W-350 \\
8 & 20.4032 & 5.7006 & 3.8441 & DU 97-W-300 \\
9 & 24.1088 & 4.6260 & 3.6252 & DU 91-W-250 \\
10 & 27.8200 & 3.9337 & 3.3909 & DU 91-W-250 \\
11 & 31.5312 & 3.6502 & 3.1683 & DU 93-W-210 \\
12 & 35.2368 & 3.4659 & 2.9458 & DU 93-W-210 \\
13 & 38.9480 & 3.0553 & 2.7232 & NACA 64-618 \\
14 & 42.6592 & 2.2913 & 2.5006 & NACA 64-618 \\
15 & 46.3648 & 1.5196 & 2.2781 & NACA 64-618 \\
16 & 49.4584 & 0.8560 & 2.0926 & NACA 64-618 \\
17 & 51.9288 & 0.4243 & 1.8872 & NACA 64-618 \\
18 & 54.4048 & 0.1084 & 1.2838 & NACA 64-618 \\
19 & 55.6400 & 0.0000 & 0.9861 & NACA 64-618 \\
\hline
\end{tabular}




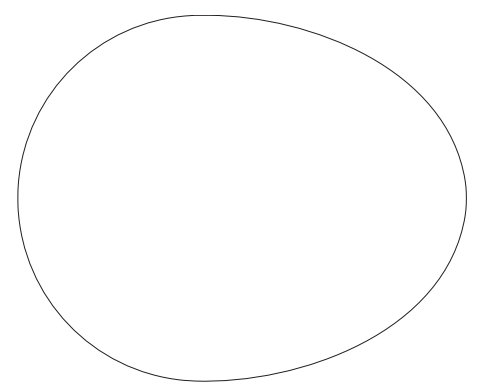

(a)

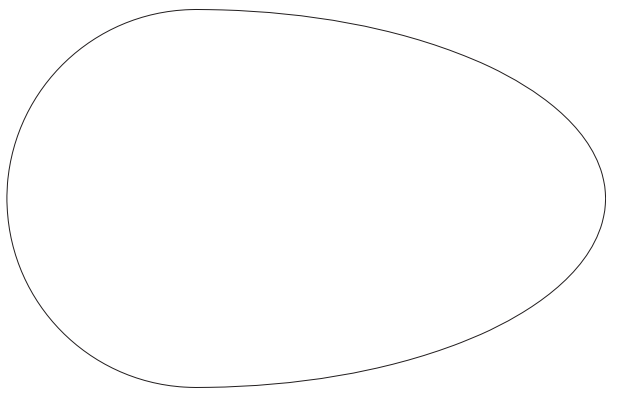

(b)

Figure 6.3. Ellipsoidal sections corresponding to: (a) station 3, and (b) station 4 on the blade span.

into a defined NACA airfoil starting from stations 5 onwards. Fig 6.3shows the profiles of these two transitional shapes.

Figure 6.4a shows us the profiles of the thick airfoils. These thick regions denote the internal structure of the airfoils located at stations 5 to 8 . These thick internal sections do help in providing a good structural rigidity to the whole blade. Aerodynamic efficiency being the highest priority for further lcoations the shape of the airfoil( as shown figure 6.4b) is more streamlined. Most of the airfoils that have been used from the root to the mid-span have D̈Uïn there denomination. DU stands for Delft University, Netherlands [64]. The DU series of airfoils were developed especially for the wind turbines of such configurations by the researchers at Delft. These blades are wildly used in the wind-turbine industry.

Aerodynamic coefficients for all the airfoils in table 6.1 are taken form [65] and [59]. The data for the cylindrical sections and the ellipsoidal sections have just the drag coefficient defined with no definition of the lift coefficient. The Cylindrical section and also the Ellipsoid-1 section has the drag coefficient to be 0.5 while the Ellipsoid-2 has a drag coefficient of 0.35 as its shape is more inclined towards a streamlined body and drifts further apart forma bluff body configuration. Figure 6.5 shows the drag, lift and momentum coefficients of all other six basic airfoil configurations of our rotor blade. This aerodynamic data is then processed with the help of Viterna's method and then corrected for rotational augmentation and is then used later on the aeroelastic simulations 5.3.1.

\subsubsection{Blade constructive aspects}

Wind turbine blade structure is in reality a combination of two external aerodynamic shells which is mounted on a box beam spar which acts as the main structural component to the aerodynamic forces. On a closer look upon any blade section we can see the aerodynamic shell along with the two spar caps. This spar caps on combining with the two 

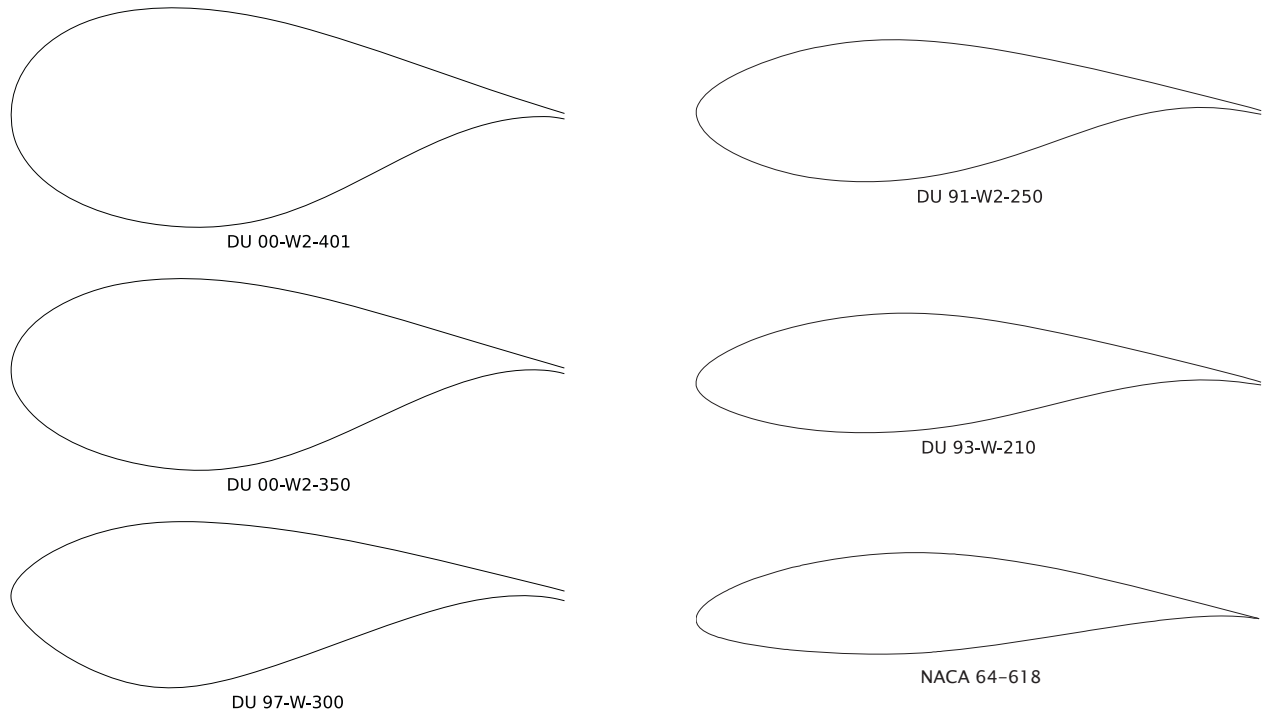

(a)

(b)

Figure 6.4. Profiles of the airfoil sections defining the blade geometry From top to bottom: (a) thick airfoils used in interior part, from station 5 to 8 of the blade-span, and (b) are the airfoils used in the mid and the tip region of the blade-span respectively. 


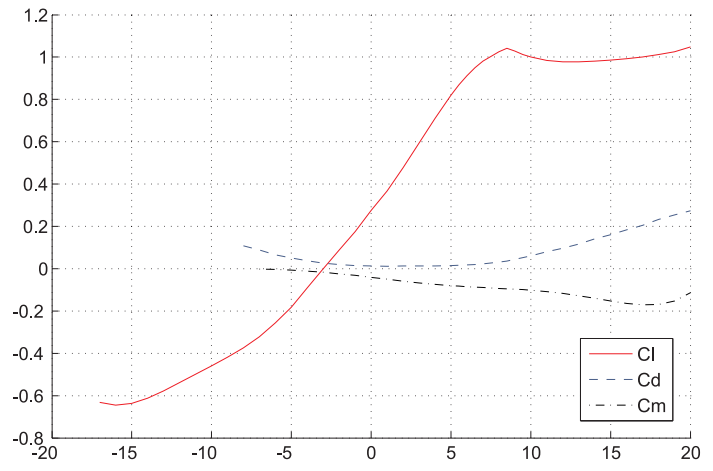

(a)

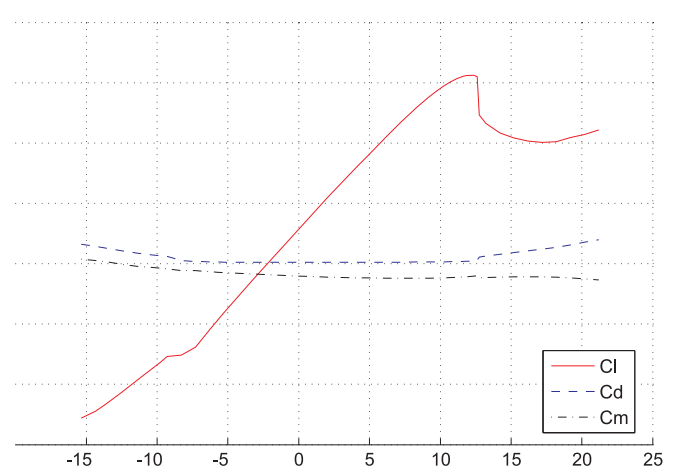

(c)

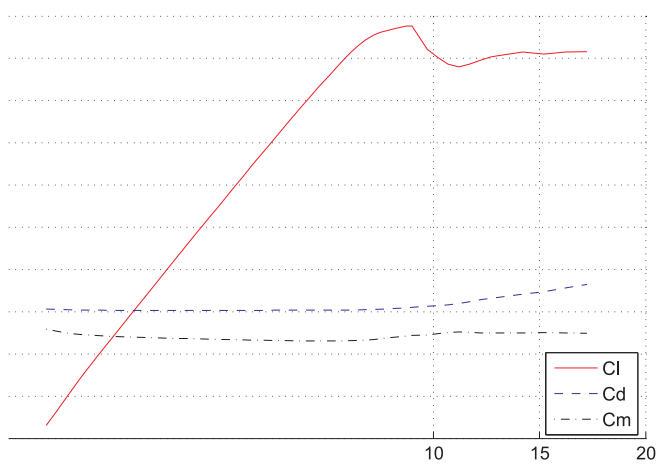

(e)

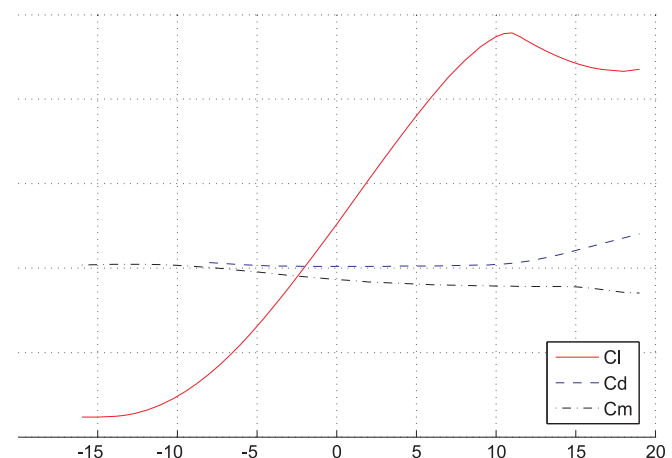

(b)

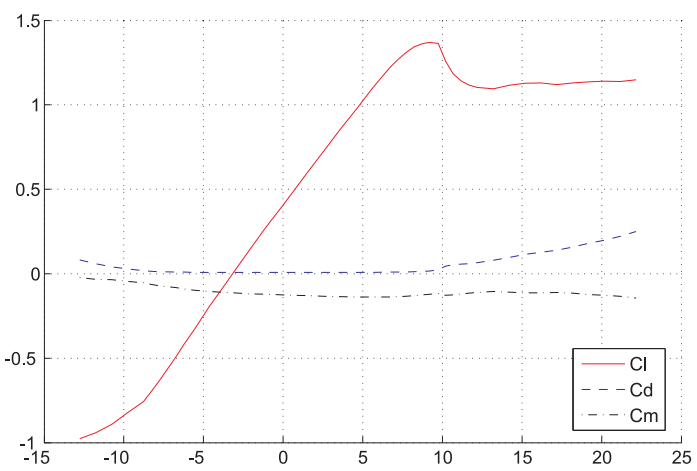

(d)

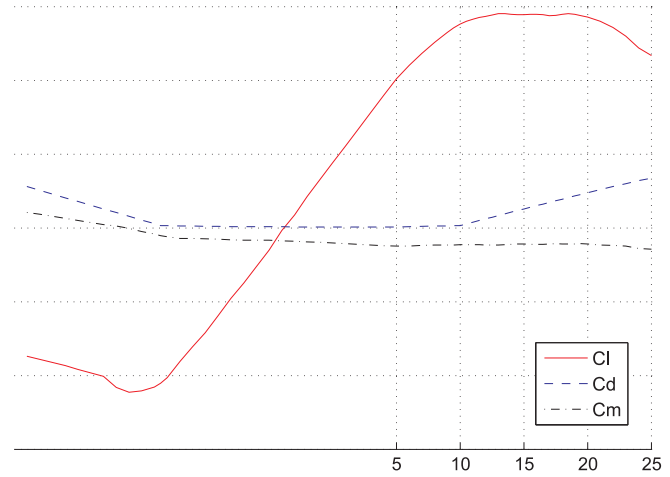

(f)

Figure 6.5. Lift, drag and momentum aerodynamic coefficients for airfoils: (a) DU 00-W-401, (b) DU 00-W-350, (c) DU 97-W-300, (d) DU 91-W-250, (e) DU 93-W-210, (f) NACA 64-618. 
vertical shear webs form the box-beam spar. Reports published by the SANDIA National labs $[19,66]$ consider a detailed description of construction of the blade in terms of thickness along with the number of layers of fiberglass required and also their orientation with respect to each other. As per the description provided in the reports these layers are made up of discrete layers of fiber glass at $\pm 45^{\circ}$ with each other along with some randomly oriented fibers, gelcoat and filler resin. The shear webs are made up of $\pm 45^{\circ}$ layers around a balsa wood core which provides the required buckling resistance during operation. The average positioning of the shear webs is at $15 \%$ and $45 \%$ along the chord of the airfoil. That being said this approximation is valid only for perfectly defined airfoils as the positioning of these shear webs in airfoils closer towards the root is different. This change in positioning of the shear webs in airfoils towards the roots helps in generating the required structural rigidity and stiffness at the root of the rotor blades. The spar caps unlike the orientation of $45^{\circ}$ are made up of layers with $0^{\circ}$ orientations. The spar caps are the most vital structural elements when it comes to those offering a substantial resistance against bending loads. A closer look at the blade section also unveils a small addition of material towards the trailing edge of the airfoil. This material filling is also made up of $0^{\circ}$ layers of glass fibers and helps in adding structural rigidity at the end of the blade. It helps in avoiding bending in chord wise directions. Report $[19,66]$ does a good job at a describing the lamination process comprehensively.

The thickness of various sections of the internal structure of the airfoil section is as per the values in Table 6.3 which in turn is obtained from [66]. The externals of the blade are covered by a $0.13 \mathrm{~mm}$ thick layer of Gelcoat. Gelcoat is a resin based paint that provides a smooth surface finishing and also shields the surface of the blade form harmful ultra violet radiations that cause degradation of the contact surface thereby reducing the aerodynamic efficiency. It also protects the destabilization of the internal layers of the blade due to exposure to the ultraviolet rays. Beneath the gelcoat can be discovered a layer of randomly oriented fibers. This layer acts as a layer that communicates the outer finishing layer with the layers of internal structure. This laminate, textitCSM (continuous strand mat) is $0.38 \mathrm{~mm}$ thick. After the layer of CSM can be found a layer of preponderant orthotropic laminate with the fibers oriented at $\pm 45^{\circ}$ with each other. These fibers are called double biased mat (DBM). The thickness of this laminate layer is given by the expression; $(A)=3,05 \times 10^{-5} L$ where $L$ is the length of the blade (6.3). These three layers remain constant along any specific cut section of the blade.

The spar caps are identical for this study to each other and are made up of three layers of laminate each. There is also a functionality to create spar caps of variable materials also variable orientations for further study if necessary. The inner most and the outer most layer of these spar caps are of thickness $2 / 3(A)$ with internal reenfocements provided by $D B M$ and running along from leading edge to the rear shear web. This constitutes about $45 \%$ of the blade section, chord wise. The mid layer being made up of $U N I$, a uniaxial laminate is what actually makes a spar cap a spar cap. Meaning, it provides the required 
Table 6.3

Structural details adapted from [66].

\begin{tabular}{|c|c|c|c|c|c|c|}
\hline $\begin{array}{l}\text { Item } \\
\text { ID }\end{array}$ & $\begin{array}{l}\text { Material De- } \\
\text { scription }\end{array}$ & $\begin{array}{l}\text { Placement Descrip- } \\
\text { tion }\end{array}$ & $\begin{array}{l}\text { Tensile } \\
\text { Modulus } \\
\text { [Msi] }\end{array}$ & $\begin{array}{l}\text { Layer } \\
\text { Width } \\
(\% \text { of } \\
\text { chord })\end{array}$ & $\begin{array}{l}\text { Layer } \\
\text { Thickness } \\
\text { [in] }\end{array}$ & $\begin{array}{l}\text { Placement Be- } \\
\text { hind L.E. (\% } \\
\text { of chord) }\end{array}$ \\
\hline 1 & Gelcoat & Outer Skin & 0.50 & $100 \%$ & 0.005 & $0 \%$ \\
\hline 2 & $3 / 4 \mathrm{oz} \mathrm{CSM}$ & Outer Skin & 1.10 & $100 \%$ & 0.015 & $0 \%$ \\
\hline 3 & DBM & Outer Skin & 1.39 & $100 \%$ & (A) & $0 \%$ \\
\hline 4 & DBM & Spar Cap Reinf. & 1.39 & $45 \%$ & $2 / 3$ of $(A)$ & $0 \%$ \\
\hline 5 & C260/520Uni & Spar Cap (at Max) & 5.41 & $30 \%$ & (B) & $15 \%$ \\
\hline 6 & DBM & Spar Cap Reinf. & 1.39 & $45 \%$ & $2 / 3$ of $(A)$ & $0 \%$ \\
\hline 7 & Balsa & Aft Panel & 0.02 & $45 \%$ & $1 \%$ & $45 \%$ \\
\hline 8 & DBM & Inner Skin & 1.39 & $100 \%$ & (A) & $0 \%$ \\
\hline 9 & $\begin{array}{l}\text { Excess } \\
\text { Resin }\end{array}$ & Inside Inner Skin & 0.50 & $100 \%$ & 0.030 & $0 \%$ \\
\hline 10 & Balsa & $35 \%$ Web Core & 0.02 & See note & $\begin{array}{l}3 \% \text { of air- } \\
\text { foil }\end{array}$ & $35 \%$ \\
\hline 11 & DBM & $35 \%$ Web Skin & 1.39 & See note & See note & $35 \%$ \\
\hline 12 & C260/520Uni & T/E spline @ 95\% & 5.41 & $6 \%$ & (C) & $92 \%$ \\
\hline 13 & TE Plexus & To 2" fwd of TE & 0.05 & $2 \%$ & fill gap & $98 \%$ \\
\hline
\end{tabular}

Notes

(A) Thousandths $=1.2 \cdot$ bladelength $50 \mathrm{~m} \rightarrow .060 "$

(B) This value is found by converging to the required flapwise moment at $3,750 \mu$

(the spar cap is twice as thick at its center as at its edges)

(C) This value is found by converging to the required edgewise moment at $1,250 \mu$

For $15 \%$ Station:

The spar cap width is $60 \%$ of chord

The spar cap begins at $5 \%$ of chord

The spar cap reinforcement ends at $65 \%$ chord

For Shear Web:

The balsa thickness was $3 \%$ of max airfoil thickness

The height was half the section height (so each half reaches center)

Skins (thousandths) $=2 \cdot$ bladelength $50 m \rightarrow 0.100$ " each skin 
Table 6.4

Baseline Blade Material Properties, as adapted from [19].

\begin{tabular}{lccccccc}
\hline Property & $E_{x}[\mathrm{GPa}]$ & $E_{y}[\mathrm{GPa}]$ & $G_{x y}[\mathrm{GPa}]$ & $\nu_{x y}$ & $\nu_{f}$ & $w_{f}$ & $\rho[\mathrm{g} / \mathrm{cm} 3]$ \\
\hline A260 & 31.0 & 7.59 & 3.52 & 0.31 & 0.40 & 0.61 & 1.75 \\
CDB340 & 24.2 & 8.97 & 4.97 & 0.39 & 0.40 & 0.61 & 1.75 \\
Glass-E & 40.0 & 8.00 & 4.00 & 0.25 & - & - & 1.90 \\
Carbon Fibre & 135.0 & 10.0 & 5.00 & 0.30 & - & - & 1.60 \\
Spar Cap Mixture & 25.0 & 9.23 & 5.00 & 0.35 & 0.40 & 0.61 & 1.75 \\
Random Mat & 9.65 & 9.65 & 3.86 & 0.30 & - & - & 1.67 \\
Balsa & 2.07 & 2.07 & 0.14 & 0.22 & N/A & N/A & 0.144 \\
Gel Coat & 3.44 & 3.44 & 1.38 & 0.30 & N/A & N/A & 1.23 \\
Fill Epoxy & 2.76 & 2.76 & 1.10 & 0.30 & N/A & N/A & 1.15 \\
\hline
\end{tabular}

structural properties of the spar cap to the spar cap. The thickness of this layer is defined as per the description provided in [66] and interpolating it for the length of our blade length. This midlayer is set up between the two vertical shear web walls making it to be present in percentages 15 to 45 of the airfoil chordwise. Thus the region of the airfoil starting from the leading edge and extending up to the first shear web will consist of the two layers that correspond to the layers reinforcing the spar cap with the absence of the third layer. Once we travel out of the spar cap zone once we go through the outer $D B M$ layer we find a balsa wood core or a solid foam layer that provides a laminar buckling stiffness to the airfoil structure. This layer is lies form the leading edge to $15 \%$ of the airfoil chord and after the spar cap region i.e. form $45 \%$ to the trailing edge. Throughout its expanse this layer exhibits a thickness matching to $1 \%$ of the airfoil chord. The interior part of the airfoil is coated with a layer of $D B M$, of $(A)$ thickness to which an extra layer of $0.76 \mathrm{~mm}$ thickness of resin is added.

The shear webs are made up of a solid foam or a balsa wood core with a thickness of $3 \%$ of the airfoil thickness. This core is applied upon by a $5 / 3(A)$ thickness of $D B M$ layer on each side and there by providing the necessary thickness. The core in turn separates the $D B M$ layers and prevents laminar buckling.

The Table 6.4 is built up upon the information obtained from the SANDIA report [19] and describes the properties of the materials that are generally used in the construction of the wind turbines. The construction of wind turbines described in this thesis make use of varying compositions of materials like balsa wood, gel coat, $U N I$ as well as epoxy resins. The properties of these materials were thus calculated with the help of table 6.4. By modifying the uniaxial laminate with various fiber orientations the properties of $D B M$ and $C S M$ were also obtained. 


\subsubsection{Blade structural properties}

The material properties described in article 6.1 .1 are the properties of the material present in each sub region of the blade, corresponding to the blade section components. These properties are assumed to be homogeneous and corresponding to those of an equivalent material. This equivalent material has a $6 \times 6$ symmetric matrix with 21 independent coefficients which were calculated by weighted average of the properties of an actual laminate. This assumption of treating the properties homogeneous dosent introduce significant errors as the thickness $f$ these regions are significantly small as compared to the length of the blade. If need be each layer can be meshed individually to obtain finer details. Separate meshing of every single layer using exact properties is also a provision that the computational codes allow.

The defining of the internal regions and materials is then followed up by meshing. A triquadrilateral mesh was generated along the span of the blade at number of blade sections. The sections present in table 6.1 served as base points for 3-D morphing. A variational cubic spline interpolation that allows us to create smooth transitions between predefined intermediate discrete numbers of 2-D airfoil sections spanning across the span of the blade was used to obtain a 3-D wind turbine as a result of 3-D morphing. The smoothness of the 3-D curve obtained is defined by the number of blade sections the blade is divided which being more than the number of predefined stations. Finer finite element meshes thus can be generated for more detailed study by merely increasing the number of sampling points. Figure 6.6 below shows us two morphed blade sections at $20 \%$ and $60 \%$ of the blade span. FE meshes for these sections along with the master sections described in table 6.1 are presented in figures 6.7 to 6.10 .

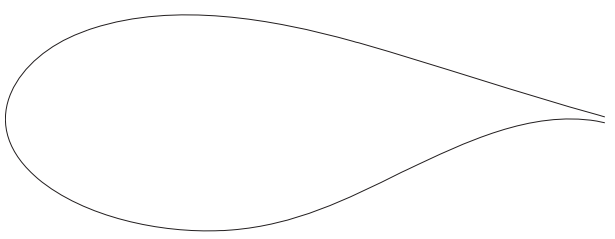

(a)

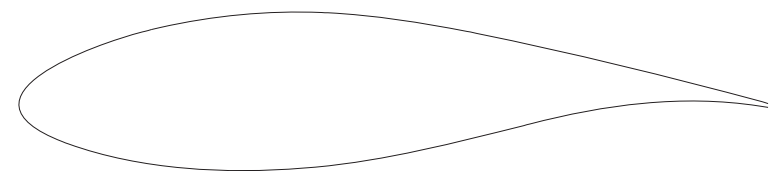

(b)

Figure 6.6. Morphed sections' profiles corresponding to: (a) $20 \%$ of the blade span, and (b) $60 \%$ of the blade span.

Following the internal blade structure components as discussed earlier 46 blade sections were defined along the span of the blade to match the ones represented in NREL [12]. The key properties that were targeted for modification were edgewise, flapwise and torsional stiffness along with the mass density at every blade section. The location of the reference points for the calculations of aerodynamic coefficients as well as the pitch axis centering were computed with the help of information available in [12]. Table 6.5 provides us with the two variables responsible for the refinement viz RefLinPos and AeroCentPos. RefLinPos 
describes the fraction of the length of the chord from the leading edge to the reference line here the longitudinal axis. For a rectilinear blade this corresponds to the pitch axis as there is no predefined deformation in the blade. AeroCentPos represents the fraction of the chord from the leading edge to the aerodynamic center of each blade section under consideration. The Location is the position of the corresponding blade section along the span of the blade measured from the root of the blade while Span is the length of the blade. Fig 6.11shows us the blade layout that is a result of the data presented in 6.5and also the positioning of the shear webs that form the internal box beam spar structure as seen in article 6.1.1. 
Table 6.5

Structural properties for the Blade section for NREL 5MW Reference Wind Turbine.

\begin{tabular}{llllll}
\hline $\begin{array}{l}\text { Location } \\
{[\mathrm{m}]}\end{array}$ & $\begin{array}{l}\text { RefLin } \\
\text { Pos }\end{array}$ & $\begin{array}{l}\text { AeroCent } \\
\text { Pos }\end{array}$ & $\begin{array}{l}\text { Flapwise } \\
\text { Stiffness }\left[\mathrm{Nm}^{2}\right]\end{array}$ & $\begin{array}{l}\text { Edgewise } \\
\text { Stiffness }\left[\mathrm{Nm}^{2}\right]\end{array}$ & $\begin{array}{l}\text { Mass Density } \\
{[\mathrm{kg} / \mathrm{m}]}\end{array}$ \\
\hline 0.00 & 0.5000 & 0.5000 & $1.81 \times 10^{10}$ & $1.81 \times 10^{10}$ & 709.73 \\
1.20 & 0.5000 & 0.5000 & $1.94 \times 10^{10}$ & $1.96 \times 10^{10}$ & 808.44 \\
4.20 & 0.4718 & 0.4435 & $1.08 \times 10^{10}$ & $1.49 \times 10^{10}$ & 619.37 \\
5.70 & 0.4383 & 0.3326 & $7.23 \times 10^{9}$ & $1.02 \times 10^{10}$ & 470.70 \\
7.20 & 0.3750 & 0.2500 & $5.53 \times 10^{9}$ & $8.06 \times 10^{9}$ & 418.81 \\
8.20 & 0.3750 & 0.2500 & $4.98 \times 10^{9}$ & $6.88 \times 10^{9}$ & 399.39 \\
11.20 & 0.3750 & 0.2500 & $3.95 \times 10^{9}$ & $7.27 \times 10^{9}$ & 435.73 \\
12.20 & 0.3750 & 0.2500 & $3.39 \times 10^{9}$ & $7.08 \times 10^{9}$ & 424.61 \\
18.20 & 0.3750 & 0.2500 & $2.05 \times 10^{9}$ & $4.50 \times 10^{9}$ & 354.73 \\
20.20 & 0.3750 & 0.2500 & $1.83 \times 10^{9}$ & $4.24 \times 10^{9}$ & 344.97 \\
22.20 & 0.3750 & 0.2500 & $1.59 \times 10^{9}$ & $4.00 \times 10^{9}$ & 336.60 \\
24.20 & 0.3750 & 0.2500 & $1.36 \times 10^{9}$ & $3.75 \times 10^{9}$ & 328.05 \\
30.20 & 0.3750 & 0.2500 & $6.81 \times 10^{8}$ & $2.73 \times 10^{9}$ & 275.29 \\
32.20 & 0.3750 & 0.2500 & $5.35 \times 10^{8}$ & $2.55 \times 10^{9}$ & 264.69 \\
38.20 & 0.3750 & 0.2500 & $2.39 \times 10^{8}$ & $1.58 \times 10^{9}$ & 209.38 \\
40.20 & 0.3750 & 0.2500 & $1.76 \times 10^{8}$ & $1.32 \times 10^{9}$ & 187.54 \\
48.20 & 0.3750 & 0.2500 & $7.63 \times 10^{7}$ & $7.10 \times 10^{8}$ & 135.43 \\
56.20 & 0.3750 & 0.2500 & $3.04 \times 10^{7}$ & $3.05 \times 10^{8}$ & 76.21 \\
59.20 & 0.3750 & 0.2500 & $1.28 \times 10^{7}$ & $1.19 \times 10^{8}$ & 54.86 \\
61.50 & 0.3750 & 0.2500 & $1.70 \times 10^{5}$ & $5.01 \times 10^{6}$ & 10.79 \\
\hline
\end{tabular}

An array of other parameters was generated considering the optimal thickness of all the consisting layers of the internal structure. These parameters are thus used to create more basis points for a morphed 3D blade. By this method it is easily possible to increase the density of a 1D mesh beyond the density available from the original data by just creating these intermediate basis points. Table 6.6 summarizes the refined structural properties exhibited by the 46 blade sections considered. The first two columns of this table are same as that of table 6.5. BMassDen is the blade mass density in $[\mathrm{kg} / \mathrm{m}]$. FlpStff, EdgStff and GLStff are flapwise stiffness, edge wise stifness and torsional stifness of the blade section. The blade section's flapwise as well as edgewise inertia of the blade section.

This blade configuration was defined by

After the blade sections refinement, we generated a library of parameters with the optimal thicknesses for the different components of the internal structure. Combining this library with our 3D-morphing technique, we are now able to increase the number of in- 
termediate blade sections at any region improving, if needed, the density of the 1-D mesh beyond the information originally reported in [12].

Table 6.6 summarizes the refined structural properties for the 46 blade sections. The first two columns are similar to those in table 6.5. BMassDen is the mass density of the blade expressed in $[\mathrm{kg} / \mathrm{m}]$. FlpStff, EdgStff and GLStff are respectively the flapwise, edgewise, and torsional stiffness for the blade section expressed in $\left[\mathrm{Nm}^{2}\right]$. Finally, flapwise, and edgewise blade section's inertia, FlpIner and EdgIner, are included, complementing the main structural properties. 
Table 6.6

Distributed blade structural properties.

\begin{tabular}{|c|c|c|c|c|c|c|c|}
\hline $\begin{array}{l}\text { Locat } \\
{[\mathrm{m}]}\end{array}$ & & $\begin{array}{l}\text { BMassDen } \\
{[\mathrm{kg} / \mathrm{m}]}\end{array}$ & $\begin{array}{l}\text { FlpStff } \\
{\left[N m^{2}\right]}\end{array}$ & $\begin{array}{l}\text { EdgStff } \\
{\left[\mathrm{Nm}^{2}\right]}\end{array}$ & $\begin{array}{l}\text { GLStff } \\
{\left[\mathrm{Nm}^{2}\right]}\end{array}$ & $\begin{array}{l}\text { FlpIner } \\
\text { [kgm] }\end{array}$ & $\begin{array}{l}\text { EdgIner } \\
{[\mathrm{kgm}]}\end{array}$ \\
\hline 0 & 0.000 & 730.232 & $1.800 \times 10^{10}$ & $1.800 \times 10^{10}$ & $4.870 \times 10^{9}$ & 1120.65 & 1120.65 \\
\hline 0.20 & 0.003 & 730.232 & $1.800 \times 10^{10}$ & $1.800 \times 10^{10}$ & $4.870 \times 10^{9}$ & 1120.65 & 120.65 \\
\hline 1.20 & 0.020 & 840.007 & $1.960 \times 10^{10}$ & $1.960 \times 10^{10}$ & $5.810 \times 10^{9}$ & 1284.93 & 284.93 \\
\hline 2.20 & 0.036 & 750.1 & 1.820 & $1.820 \times 10^{10}$ & $5.300 \times 10^{9}$ & 1183.36 & 1183.54 \\
\hline 3.20 & 0.052 & 778.927 & $1.540 \times 10^{10}$ & $1.980 \times 10^{10}$ & $3.570 \times 10^{9}$ & 1.595 & 1317.44 \\
\hline 4.20 & 0.068 & 585.288 & $1.070 \times 10^{10}$ & $1.460 \times 10^{10}$ & $2.550 \times 10^{9}$ & 863 & 1000.13 \\
\hline 5.20 & 0.085 & 438.927 & $7.330 \times 10^{9}$ & $1.010 \times 10^{10}$ & $1.940 \times 10^{9}$ & & 729.300 \\
\hline 6.20 & 0.101 & 431.718 & $6.420 \times 10^{9}$ & $9.260 \times 10^{9}$ & $1.820 \times 10^{9}$ & & \\
\hline 7.20 & 0.117 & 30 & $5.860 \times 10^{9}$ & $8.240 \times 10^{9}$ & $1.490 \times 10^{9}$ & & \\
\hline 8.20 & 0.133 & 404.740 & $4.980 \times 10^{9}$ & $6.860 \times 10^{9}$ & $1.110 \times 10^{9}$ & 329.072 & 526.006 \\
\hline 9.20 & 0.150 & 435.733 & $4.760 \times 10^{9}$ & $6.860 \times 10^{9}$ & $6.160 \times 10^{8}$ & 289.528 & 476.776 \\
\hline 10.20 & 0.166 & 487.452 & $4.560 \times 10^{9}$ & $6.850 \times 10^{9}$ & $4.810 \times 10^{8}$ & 273.153 & 473.181 \\
\hline 11.20 & 0.182 & 458.237 & & $6.950 \times 10^{9}$ & & 233.322 & 480.004 \\
\hline 12.20 & 0.198 & 429.125 & $3.340 \times 10^{9}$ & $6.480 \times 10^{9}$ & $3.840 \times 10^{8}$ & 61 & 869 \\
\hline 13.20 & 0.215 & 406.951 & $2.930 \times 10^{9}$ & $6.140 \times 10^{9}$ & $3.830 \times 10^{8}$ & 39 & 449.523 \\
\hline 14.20 & 0.231 & 3 & $2.590 \times 10^{9}$ & $5.300 \times 10^{9}$ & $3.690 \times$ & 06 & .022 \\
\hline 15.20 & 0.247 & 372.182 & $2.440 \times 10^{9}$ & $5.240 \times 10^{9}$ & 4.110 & 88 & 418.817 \\
\hline 16.20 & 0.263 & 364.970 & $\times 10^{9}$ & $4010 x$ & 4.150 & 31 & 395.703 \\
\hline 18.20 & 0.296 & 350.502 & $\times 10^{9}$ & $4.540 \times 10^{9}$ & $3.880 \times 10^{8}$ & 135 & 372.744 \\
\hline 20.20 & 0.328 & 335.676 & $1.890 \times 10^{9}$ & $4.220 \times 10^{9}$ & $2.590 \times 10^{8}$ & 117.714 & 321.271 \\
\hline 22.20 & 0.361 & 340.473 & $1.570 \times 10^{9}$ & $4.230 \times 10^{9}$ & $1.790 \times 10^{8}$ & 95.670 & 305.628 \\
\hline 24.20 & 0.394 & 355.369 & $1.370 \times 10^{9}$ & $3.730 \times 10^{9}$ & $1.120 \times 10^{8}$ & 81.234 & 258.088 \\
\hline 26.20 & 0.426 & 338.738 & $9.980 \times 10^{8}$ & $3.400 \times 10^{9}$ & $8.960 \times 10^{7}$ & 59.287 & 234.431 \\
\hline 28.20 & 0.459 & 310.922 & $8.220 \times 10^{8}$ & $3.070 \times 10^{9}$ & $9.920 \times 10^{7}$ & 49.587 & 217.998 \\
\hline 30.20 & 0.491 & 284.503 & $\times 10^{8}$ & $2.720 \times 10^{9}$ & $1.080 \times$ & 41.455 & 200.583 \\
\hline 320 & 0.524 & 263.924 & $5.310 \times 10^{8}$ & $2.450 \times 10^{9}$ & $8.750 \times 10^{7}$ & 32.762 & 178.544 \\
\hline 34.20 & 0.556 & 269.853 & $4.030 \times 10^{8}$ & $2.310 \times 10^{9}$ & $7.480 \times 10^{7}$ & 25.017 & 169.907 \\
\hline 3. 20 & 0.589 & 233.829 & $3.150 \times 10^{8}$ & $1.830 \times 10^{9}$ & $6.530 \times 10^{7}$ & 19.794 & 138.727 \\
\hline & & & & $1.530 \times$ & $6.940 \times 10^{7}$ & 15.969 & 125.554 \\
\hline 40.20 & 0.654 & 190.141 & $1.760 \times 10^{8}$ & $1.320 \times 10^{9}$ & $6.610 \times 10^{7}$ & 12.398 & 115.712 \\
\hline 42.20 & 0.686 & 177.600 & $1.260 \times 10^{8}$ & $1.160 \times 10^{9}$ & $4.890 \times 10^{7}$ & 8.842 & 99.078 \\
\hline 44.20 & 0.719 & 163.609 & $1.040 \times 10^{8}$ & $1.010 \times 10^{9}$ & $3.970 \times 10^{7}$ & 7.270 & 84.880 \\
\hline 46.20 & 0.751 & 147.221 & $9.100 \times 10^{7}$ & $7.970 \times 10^{8}$ & $3.040 \times 10^{7}$ & 6.181 & 65.809 \\
\hline 48.20 & 0.784 & 132.601 & $7.570 \times 10^{7}$ & $6.910 \times 10^{8}$ & $2.410 \times 10^{7}$ & 5.098 & 55.642 \\
\hline 50.20 & 0.816 & 113.774 & $6.100 \times 10^{7}$ & $5.180 \times 10^{8}$ & $1.830 \times 10^{7}$ & 4.071 & 41.930 \\
\hline
\end{tabular}

Continued on next page... 
Table 6.6 - Continued

\begin{tabular}{llllllll}
\hline $\begin{array}{l}\text { Location Span } \\
{[\mathrm{m}]}\end{array}$ & & $\begin{array}{l}\text { BMassDen FlpStff } \\
{[\mathrm{kg} / \mathrm{m}]}\end{array}$ & {$\left[\mathrm{Nm}^{2}\right]$} & $\begin{array}{l}\text { EdgStff } \\
{\left[N \mathrm{~m}^{2}\right]}\end{array}$ & $\begin{array}{l}\text { GLStff } \\
{\left[N m^{2}\right]}\end{array}$ & $\begin{array}{l}\text { FlpIner } \\
{[\mathrm{kgm}]}\end{array}$ & $\begin{array}{l}\text { EdgIner } \\
{[\mathrm{kgm}]}\end{array}$ \\
\hline 52.20 & 0.849 & 104.913 & $5.050 \times 10^{7}$ & $4.390 \times 10^{8}$ & $1.570 \times 10^{7}$ & 3.395 & 35.788 \\
54.20 & 0.881 & 95.769 & $3.910 \times 10^{7}$ & $4.020 \times 10^{8}$ & $1.400 \times 10^{7}$ & 2.713 & 32.817 \\
55.20 & 0.898 & 88.635 & $3.450 \times 10^{7}$ & $3.530 \times 10^{8}$ & $1.200 \times 10^{7}$ & 2.378 & 28.583 \\
56.20 & 0.914 & 80.062 & $3.020 \times 10^{7}$ & $2.990 \times 10^{8}$ & $9.500 \times 10^{6}$ & 2.043 & 23.813 \\
57.20 & 0.930 & 76.518 & $2.580 \times 10^{7}$ & $2.730 \times 10^{8}$ & $8.400 \times 10^{6}$ & 1.759 & 21.572 \\
57.70 & 0.938 & 70.728 & $2.360 \times 10^{7}$ & $2.470 \times 10^{8}$ & $6.240 \times 10^{6}$ & 1.551 & 18.559 \\
58.20 & 0.946 & 63.750 & $1.980 \times 10^{7}$ & $1.560 \times 10^{8}$ & $5.900 \times 10^{6}$ & 1.333 & 13.153 \\
58.70 & 0.954 & 58.966 & $1.540 \times 10^{7}$ & $1.380 \times 10^{8}$ & $5.400 \times 10^{6}$ & 1.072 & 11.849 \\
59.20 & 0.963 & 54.574 & $1.270 \times 10^{7}$ & $1.150 \times 10^{8}$ & $3.880 \times 10^{6}$ & 0.863 & 9.473 \\
59.70 & 0.971 & 52.417 & $9.820 \times 10^{6}$ & $1.010 \times 10^{8}$ & $2.970 \times 10^{6}$ & 0.665 & 8.034 \\
60.20 & 0.979 & 49.277 & $7.230 \times 10^{6}$ & $8.370 \times 10^{7}$ & $1.680 \times 10^{6}$ & 0.469 & 6.091 \\
60.70 & 0.987 & 41.763 & $2.460 \times 10^{6}$ & $3.100 \times 10^{7}$ & $1.610 \times 10^{6}$ & 0.209 & 3.100 \\
\hline
\end{tabular}

\subsubsection{Turbine specifications}

For the purpose of validation, the general specifications of the turbine also match the ones on NREL's report [12], which are summarized in table 6.7. Thus, the rotor has an upwind orientation and it is composed of three $61.5 \mathrm{~m}$ long blades. The hub diameter is $3 \mathrm{~m}$ and it is located at $90 \mathrm{~m}$ from ground level. Total rotor diameter is $126 \mathrm{~m}$. It has a precone of $2.5^{\circ}$ and an overhang distance of $5 \mathrm{~m}$ from the tower axis. The rated wind speed for this turbine is $11.4 \mathrm{~m} / \mathrm{s}$. 
Table 6.7

Global turbine parameters

\begin{tabular}{lr}
\hline Description & Value \\
\hline Rating & $5 \mathrm{MW}$ \\
Rotor Orientation & Upwind \\
Configuration & $3 \mathrm{blades}$ \\
Rotor, hub diameter & $126 \mathrm{~m}, 3 \mathrm{~m}$ \\
Hub Height & $90 \mathrm{~m}$ \\
Rated wind speed & $11.4 \mathrm{~m} / \mathrm{s}$ \\
Rated rotor speed & $12.1 \mathrm{rpm}$ \\
Overhang & $5 \mathrm{~m}$ \\
Rotor precone & $2.5^{\circ}$ \\
\hline
\end{tabular}




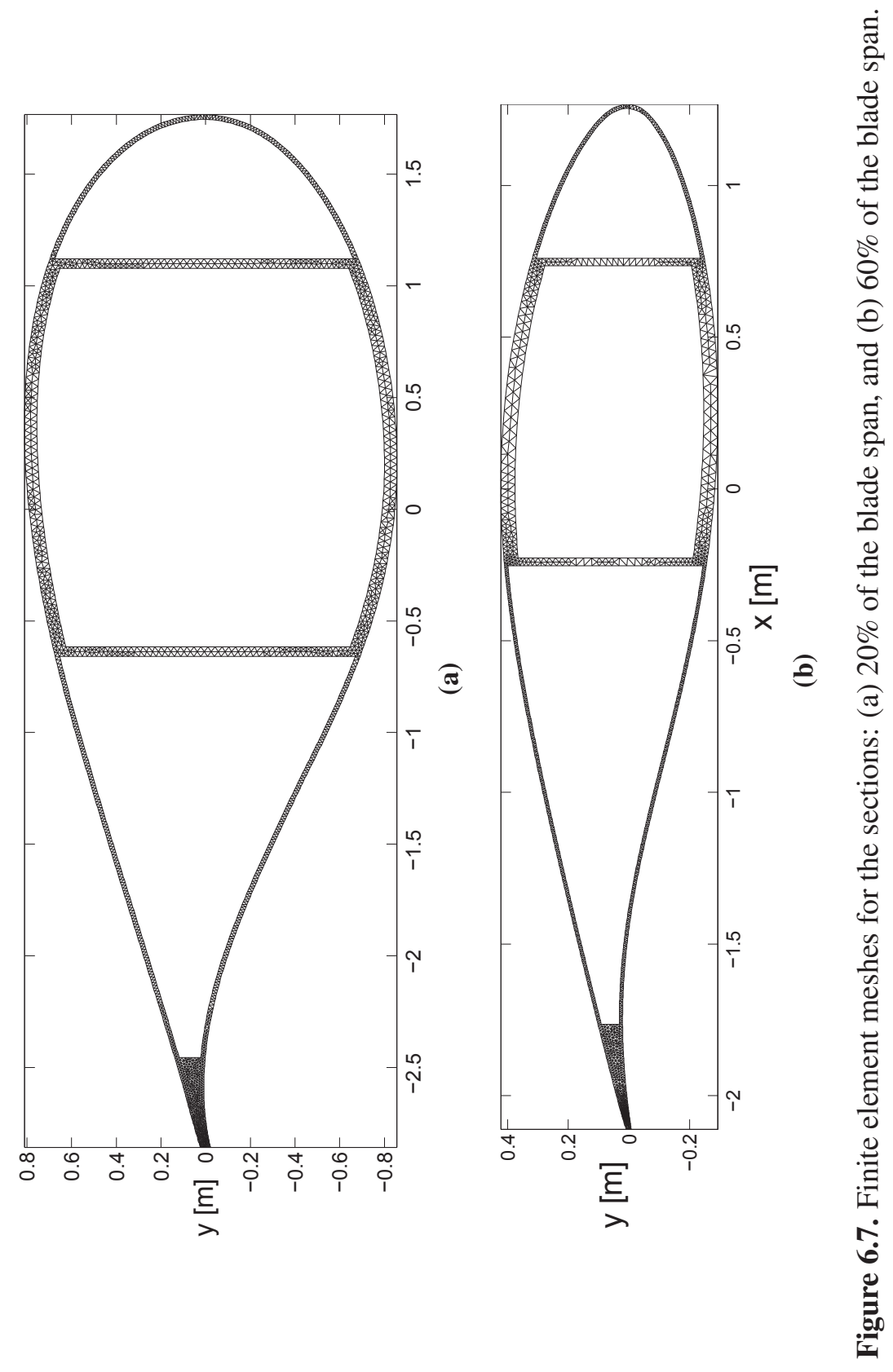




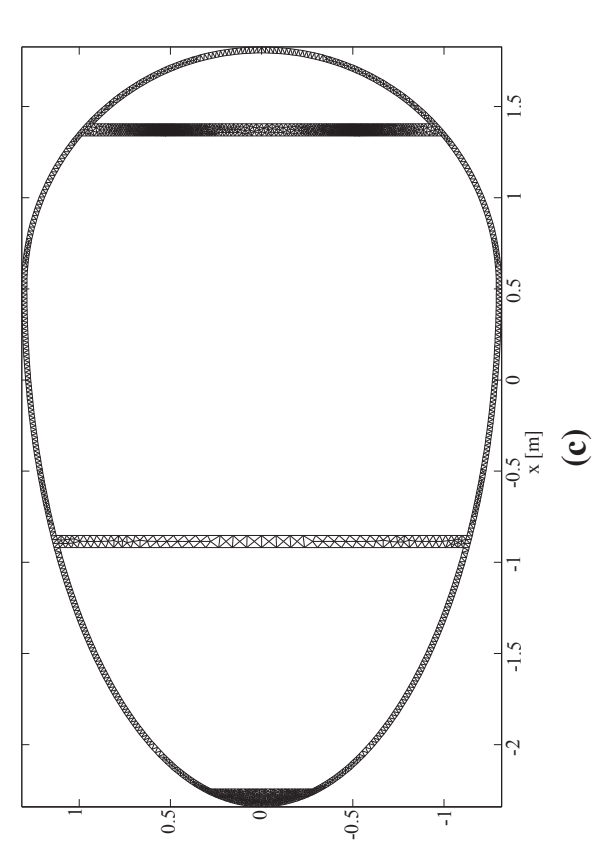

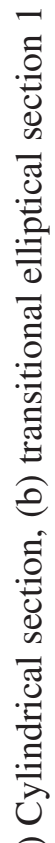

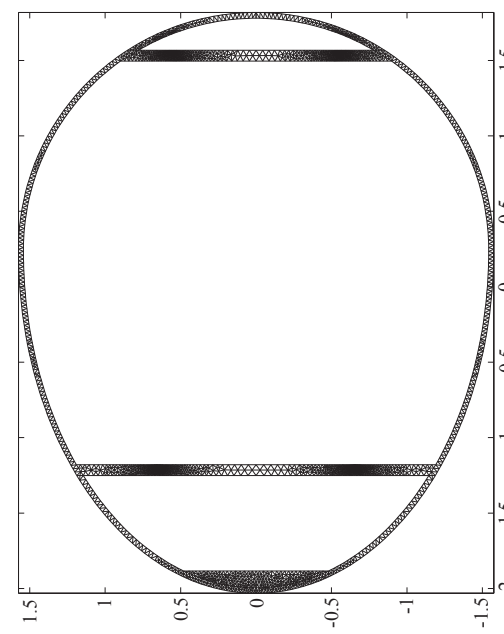

(

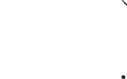



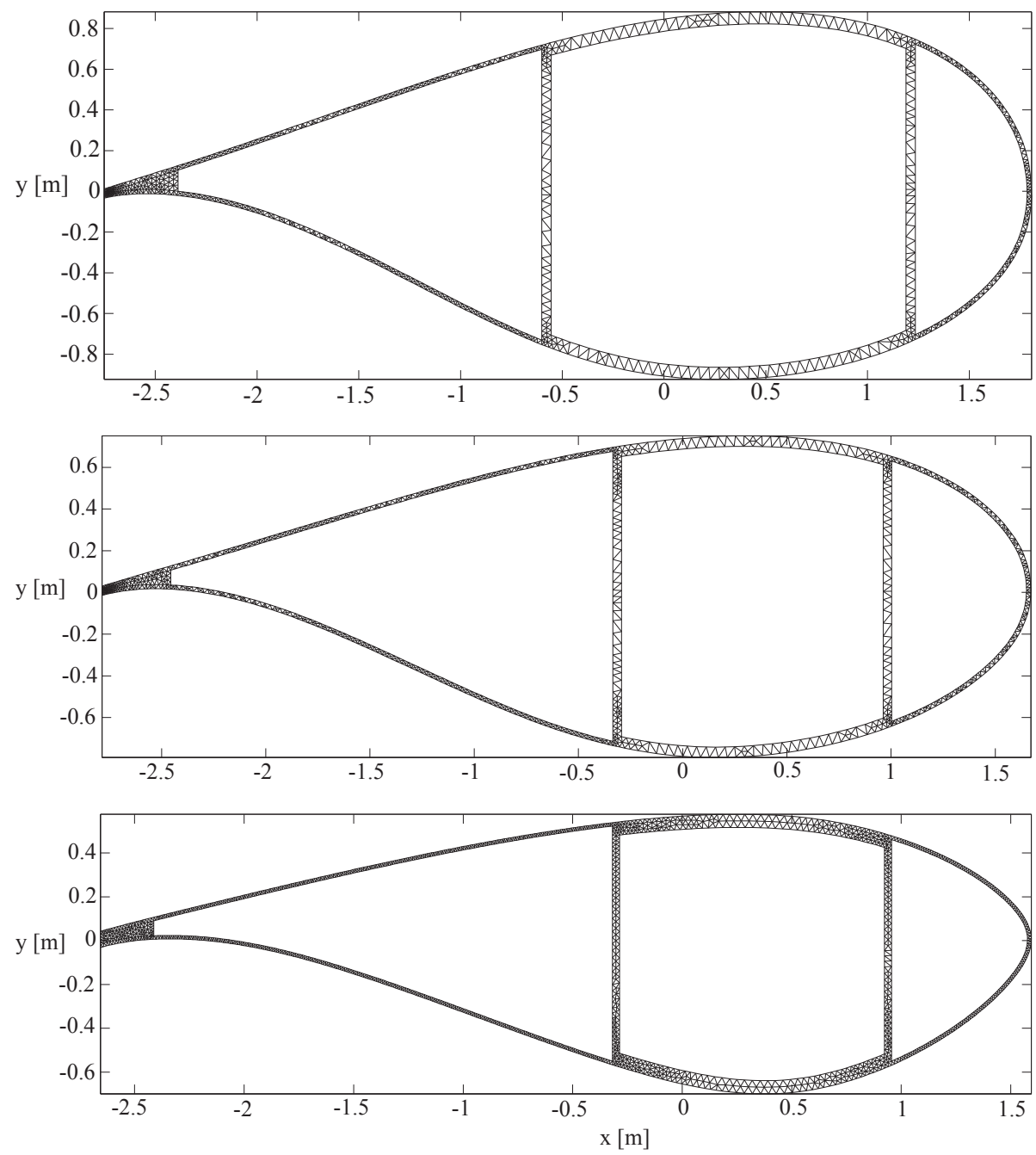

Figure 6.9. Finite element meshes for the master sections of the inner blade region. From top to bottom, DU 00-W-401, DU 00-W-350, DU 97-W-300. 

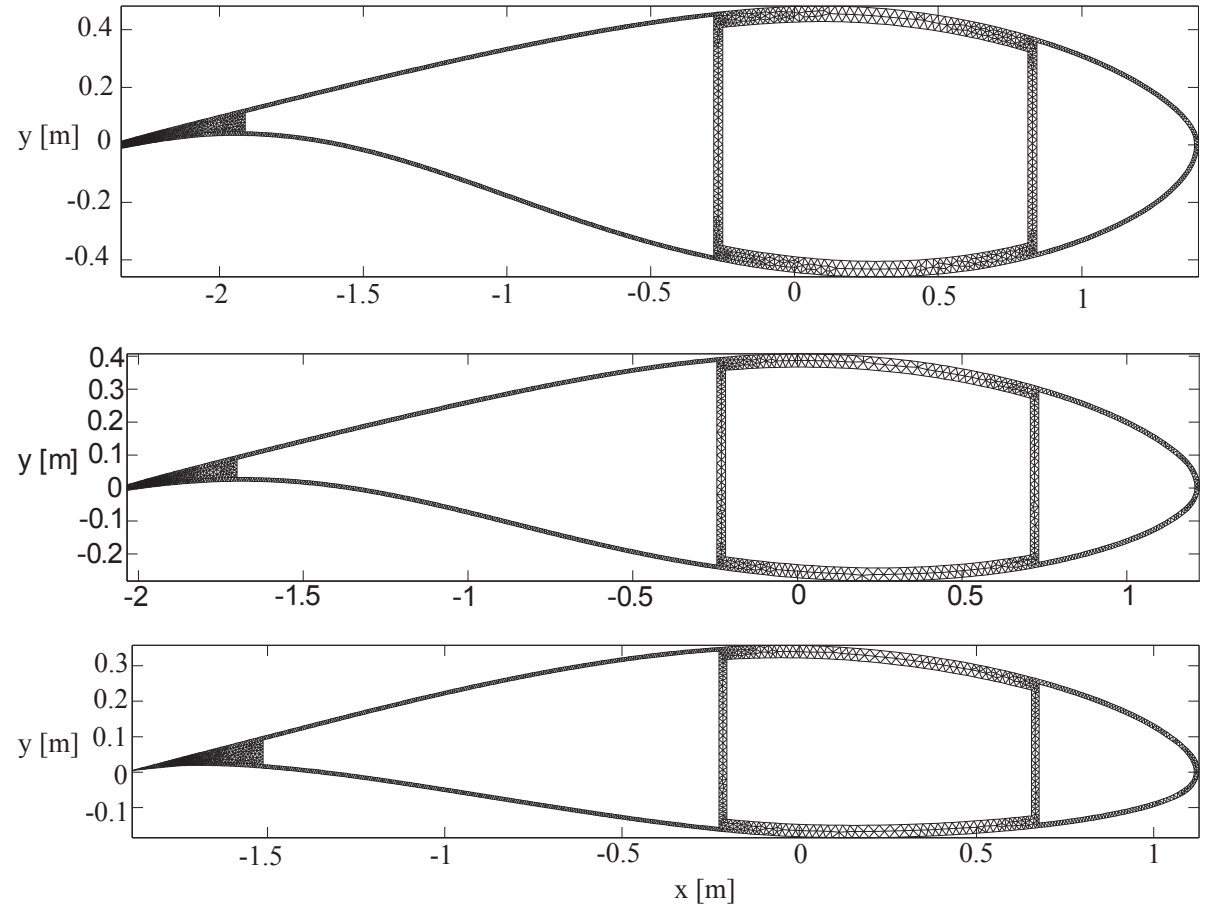

Figure 6.10. Finite element meshes for the master sections of the outer blade region. From top to bottom, DU 91-W-250, DU 93-W-210, NACA 64-618. 


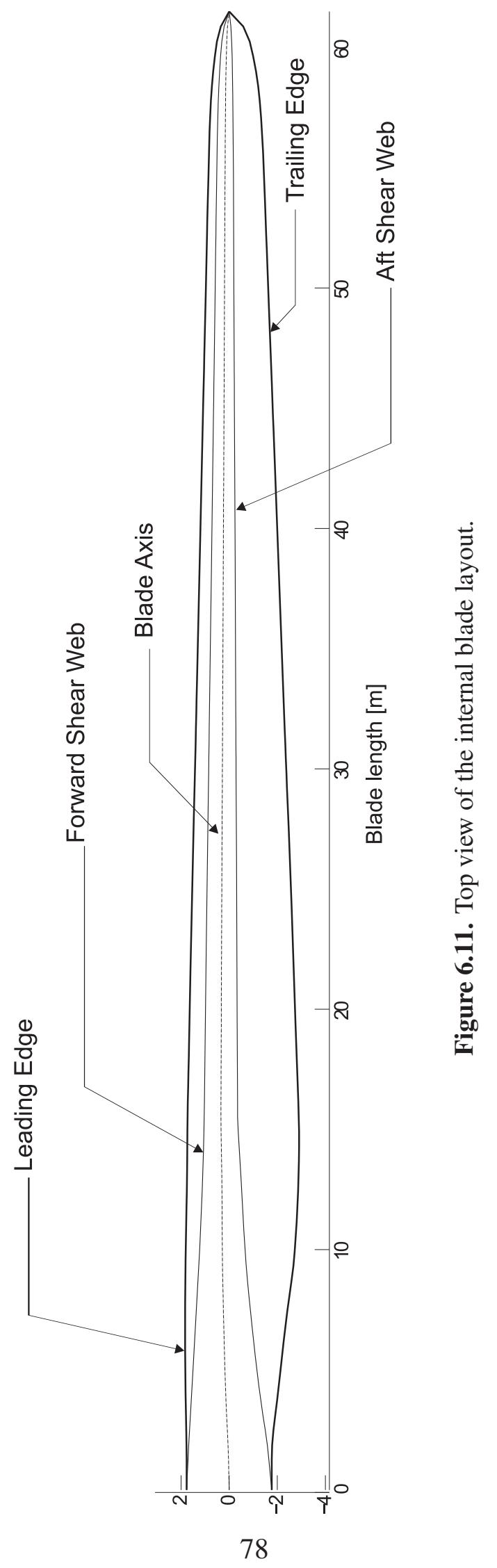




\subsection{Validation Tests}

Dimensional reduction method as discussed in 4.2 was used after the stiffness and inertia matrices were calculated at each cross section. LAMBDA the tip speed ratio for nominal operating conditions was taken to be 7 making the tangential velocity at the tip to be 80 $\mathrm{m} / \mathrm{s}$. The validation tests for steady state analysis were carried out for wind speeds of 11.4 , $12,13,14,15,17$, and $25 \mathrm{~m} / \mathrm{s}$.

In this section we will show a series of validation tests. We will start by presenting a steady-state solution around the nominal working condition, including the vibrational modes obtained from an eigenvalue analysis of a linearized solution around the steadystate. Next, we will test our model under an acceleration ramp simulating an arbitrary start-up from zero angular velocity to nominal operation conditions on the rotor. Finally, a computation of the blade-pitch angle for power-control over high wind speeds will be presented.

\subsubsection{Blade Cases}

More than 25 cases simulating different blades some completely independent while some more or less like a family of blades were run for the same problem definition in order to obtain comparable results. Out of all the experiments the blades described in this dissertation is limited to seven. On our way in explaining these seven blades their predecessors as well as their derivatives are also explained shortly.

\subsubsection{Original Blade}

Let us first start with our stock original blade as this would set a datum for our comparison. The original blade was made up of standard configurations as described earlier with a nine part internal structure. Also as discussed earlier the power output is seen to be more or less similar to the one provided by the NREL report []. Wind turbine blades as airplane blades work on the principle of lift. The air flowing over the wing generates a force called as the LIFT which is the driving force of these blades. Unlike airplanes where the relative velocity of the airfoil at each blade section is same the relative velocity is directly proportional to the distance of the blade section from the rotor. The blade sections are so designed and placed that the incident wind velocity is always directed tangentially to surface of the blade and pointing to the trailing edge. If this incident velocity is not channeled properly the lift force will take its toll on the generator. As we know by the turbine architecture, the rotational velocity of the blades is what drives the generator. The aim here is to make the power output as flat as possible. A smooth relative velocity profile generates that. As seen 
from the power plot below the blade power output keeps on increasing with a positive linear gradient until the wind speed marks $15 \mathrm{~m} / \mathrm{s}$. This implies a constant increase in lift along the blade sections. Increase in lift in turn states separated flow. After $15 \mathrm{~m} / \mathrm{s}$ we can see that the slope has decreased in its value a more flat power output is observed with the same trend continuing until the wind speed reaches the $20 \mathrm{~m} / \mathrm{s}$ mark. For the current configuration taking the material adaptiveness into consideration the flow still gets separated further and the blade can be seen to enter a stalled region reflected by less linear rise in the power output with further increased speeds. Pitch control from the rotor root is an active method of controlling the lift by changing the angle of attack. All the characteristics exhibited by this blade are solely due the structural changes and material adaptiveness 6.12.

\subsubsection{Superblade}

Next step in blade sampling was design of the superblade. The superblade has the exact inner skeleton of the original blade. The difference lies in the material that constitutes each layer. For the superblade the layer of DBM (double bias mat) was replaced by UNI, a uniaxial laminate. Also the shear web material was changed from DBM to UNI. The results speak for themselves as seen in the power output plot exhibited below6.12. The blade with changed material arrangement and with few substitutions starts with a lower power output at the nominal speed of $11.4 \mathrm{~m} / \mathrm{s}$. The power output seen is $4.633 \mathrm{MW}$ which is a megawatt less than the indicated power output for an NREL turbine. The power output line runs parallel to the power output of the original blade but with a lower magnitude. A power output of $8.755 \mathrm{MW}$ is seen to $9.39 \mathrm{MW}$ of the original blade at the speed of $15 \mathrm{~m} / \mathrm{s}$. This shows how the superblade as adapted itself in generating a controlled lift and thereby curbing the power. When the original blade shows a decrease in slope after the $15 \mathrm{~m} / \mathrm{s} \mathrm{mark}$ the superblade sticks to its curve and the power output curve can be seen to continue in the same trend as it did form its inception. This shows how the superblade is still maintaining a connected flow by maintaining the angle of attacks around its optimum values. This is achieved by the pseudo-active pitch control exhibited by material adaptiveness. When a stiff blade enters into stall at such high speeds the superblade feathers itself into the wind flow and thereby maintaining the connected flow ultimately keeping the lift in check. At the $20 \mathrm{~m} / \mathrm{s}$ mark when the most of the original blade enters stall zone which drastically reduces its power output, the super blade is seen to generate more power with just a slight decrease in gradient. This proves the material adaptiveness of a soft blade. The power output is increased with the increase in lift however there is no abrupt changes in the power curve reflecting how beautifully the blade has adapted itself to the increased speed of the air flow while still maintaining the angle of attack to generate increased power output. Unfortunately running the generators at such high speed is not desirable and even thought eh superblade excels aerodynamically by the virtue of material adaptiveness we cannot use it at higher RPMs. None the less, the superblade does a good job at explaining the effects 
of pseudo-active pitch control due to material adaptiveness.

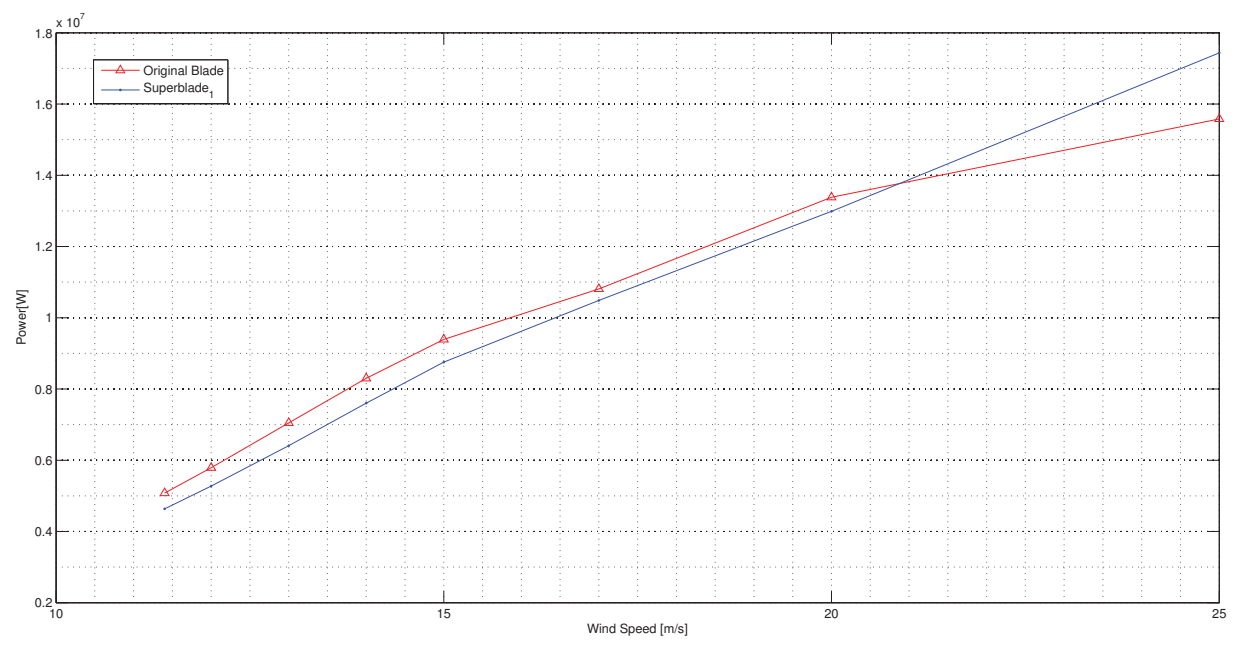

Figure 6.12. Power output of Original and Superblade.

\subsubsection{EGGshell}

After seeing the clear effects of the material adaptiveness on the blade we came with a concept of three blades viz eggshell, Utube and Combo blade. All these blades were built keeping the superblade as their base model. We came up with a material hinge which would target specific areas pf the blade along its span. The hinge was located on strategic locations based on engineered guess and study of the behavior of HAWT blades. For the eggshell the hinge created was the one varying from its full capacity to $20 \%$ of its capacity. The hinge created was active for a part from $15 \%$ to $40 \%$ of the blade span with the distance measured from the root to the tip of the blade. This hinge would change the thickness of the UNI layer for all selected regions and bring the thickness down to $20 \%$ of the original form $15 \%$ blade length to $40 \%$ of the same. This hinge for the eggshell was applicable for all regions excluding both the shear webs and the trailing edge. This hinge was active on the outer surface of the blade symbolizing an egg and hence the name. The blade becomes softer at parts where the hinge is located. It is termed as a hinge as both sides of the hinge are more or less are subject to the aerodynamic forces and are not held back by blade rigidity. However when we go down on observing the power output of both the superblade and compare it to the eggshell which is but just a modified version of it, the results are similar with just a slight changes. If observed closely the Eggshell ends up giving out more power output at higher wind seeds. Thus it can be well concluded that the eggshell is more or less similar to the eggshell exhibiting the similar trends as that of the eggshell. 
Further evaluation of the eggshell involved change the hinge location and also the reach of the hinge. At the end of the day all permutations of the eggshell reflected closely similar results. EggTwist_25 was an upgraded version of eggshell blade. EggTwist_ 25 was made up of two hinges, first being the same hinge as we came across in the eggshell. The second hinge was a hinge that was applicable on the manufacturing angles of the fibers. It was an inverted hinge working at $25 \%$ intensity till $15 \%$ of the blade span and from $15 \%$ to $40 \%$ of the blade span worked on its $100 \%$. The second hinge changed the fiber orientation on the spar caps as per the hinge intensity. Thus the manufacturing angle was 20 degrees for the top spar cap and -20 for spar at bottom in its original configuration and was changed to $20 \times 0.25=5$ for the regions excluding the hinge span and restored back to 20 for the rest of the blade span. This blade showed same characteristics as that of the superblade and the eggshell but at a higher magnitude with increased wind speeds. The superblade showed a power output of $8.755 \mathrm{MW}$ and $12.99 \mathrm{MW}$ at wind speeds of $15 \mathrm{~m} / \mathrm{s}$ and $20 \mathrm{~m} / \mathrm{s}$ respectively as opposed to the power outputs of $8.967 \mathrm{MW}$ and 13.27MW exhibited by Eggtwist_25 for the same wind speeds. On the lines of eggshell were designed two more blades, one with the same material hinge active for just the spar caps and the nose (assuming the shape $U$ ) while the other one being a boolean of both, we termed it the COMBO blade. Both these blades exhibited same trends as their mother blade, the superblade with minor differences 6.13 .

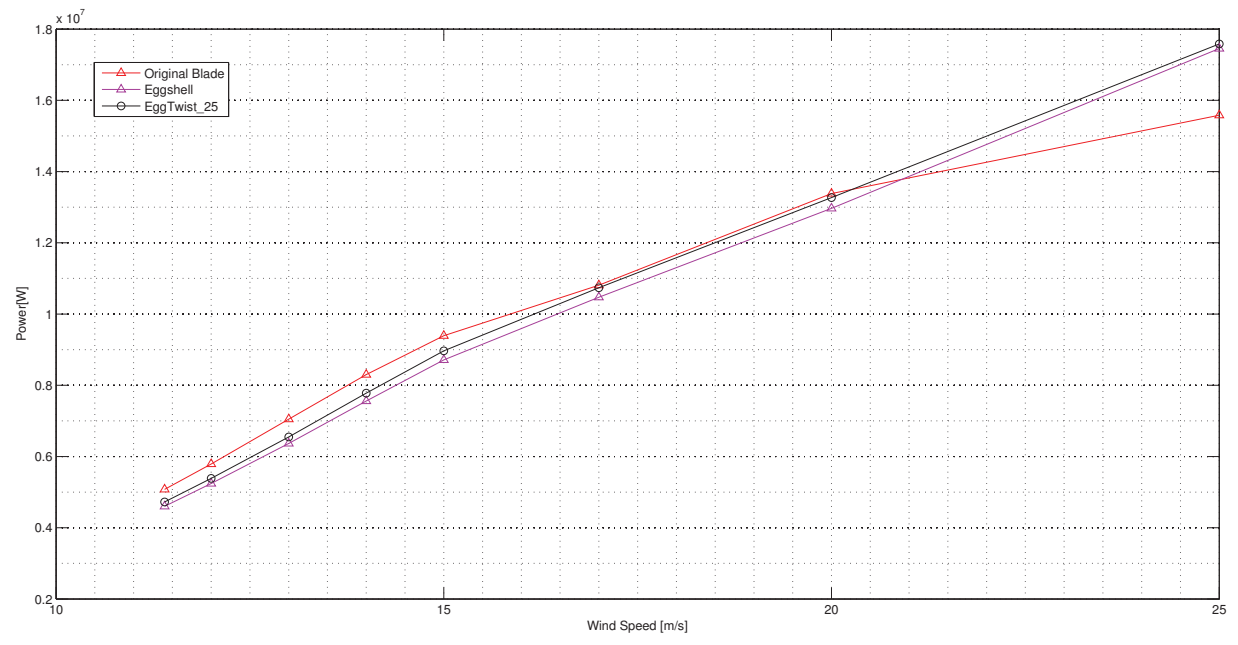

Figure 6.13. Power output of Original and EGGshell. 


\subsubsection{HingeTwist_75_Combo}

HingeTwist_ 75_Combo is yet another two hinged materially adaptive wind turbine a modification of the combo blade as discussed earlier. Similar to that of the eggshell's two hinged modified version, HingeTwist _ 75_ Combo also showed similar trends as that of its one hinged predecessor. The reason this blade is so important is that it paved a new path for other more materially as well as geometrically adaptive blades. The characteristics shown HingeTwist_ 75_Combo even though similar to that of the Combo blade are lesser in magnitude than that later. This shows how the hinge tinkering with the fiber orientation curbs down the power for same operating conditions6.14.

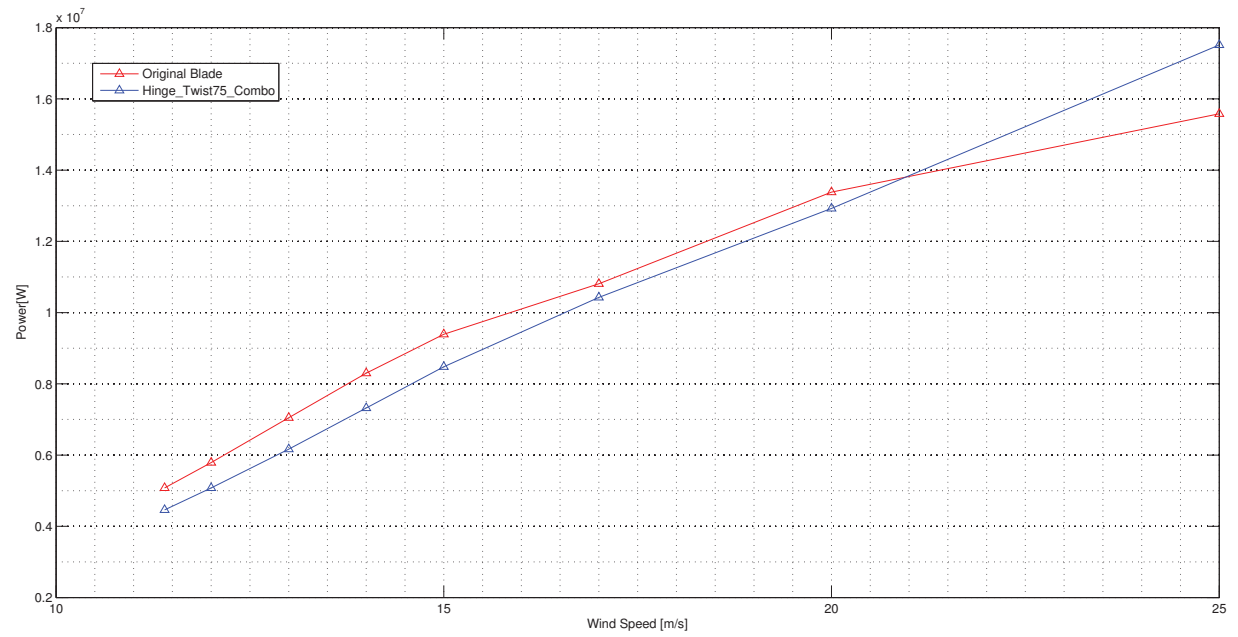

Figure 6.14. Power output of Original and HingeTwist_75_Combo.

\subsubsection{Sweptback blades}

Sweptback blades With considerable effects on the blades behavior shown by material adaptiveness we now move on geometrical adaptiveness by introducing swept back blades. Sweptback blades are once whose Reference line starts at the rotor and translates to end up inclining towards the tower. Due to the limited distance available between the blade reference line and the tower there is only a limited scope for building sweptback blades. It is evident from its behavior that the swept back blade sticks to connected flow and feathers itself in the wind flow to avoid flow separation. Hence including swept back blades and fusing it with material adaptiveness promised positive results 6.15 . 


\subsubsection{Stall blades}

This stall blade results were obtained from the research experiments done by Nimish Deshpande. The blade used in this analysis was a fixed speed stall controlled blade (DELFT). The part of the blade that operates in stall region varies drastically in its geometric structure. These stall creations tend to impart large stresses on the blade subsequently raking its toll on the blade durability. In order to induce stall in regions further away from the root of the blade the twists in these sections of the blade was reduced by $70 \%$ of the twist of the original blade. The standard working conditions were changed to $10.7 \mathrm{rpm}$ with the nominal wind speed of $15.31 \mathrm{~m} / \mathrm{s}$. This change in was done as the data form experimentation wasn't available for wind-speeds below $15.31 \mathrm{~m} / \mathrm{s}$. This speed of $15.31 \mathrm{~m} / \mathrm{s}$ was obtained from the alternative design relation [ref]. As seen in the power plot fig 6.15 the stall blades shows a more flat power curve and behaves as it was anticipated to be. At the wind speed of $14 \mathrm{~m} / \mathrm{s}$ where original blade was showing a power output of $8.3 \mathrm{MW}$ the stall blade gives out a power of $5.675 \mathrm{MW}$. At this point it became evident that feathering blades were showing desirable behavior for lower wind speeds while the stall blades were behaving excellently in the higher wind speed domain.

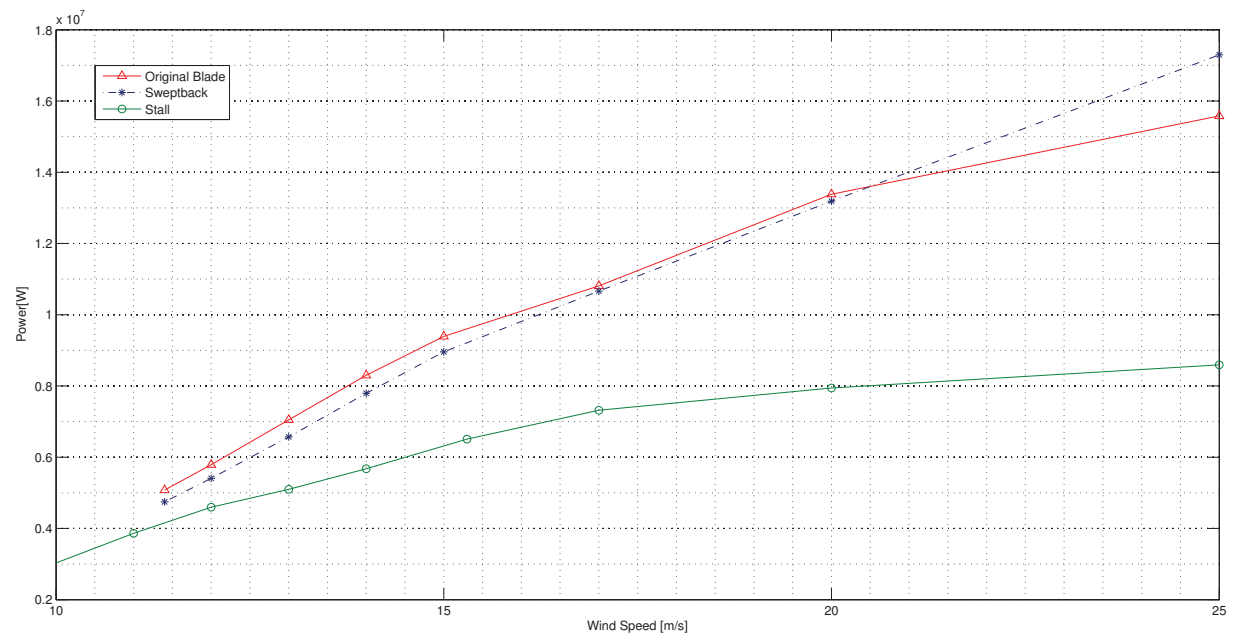

Figure 6.15. Power output of Original,Sweptback, and Stall blades.

We then decided to merge both these concepts to churn out a multi concept smart blade that provided the best of both worlds. Numerous numerical experimentations were done and then final two blades which showed promising results were chosen. Those two blades were Hinge_ Twist_ 75_Combo_ Stall and Hinge_ Twist_ 75_Combo_ Sweptback. As discussed earlier the two material hinge design showed promising result when it came down 
to material adaptiveness. The sweptback blade with its geometrical adaptations showed a broad spectrum of connected flow regime. The stall blades on the other hand with a whole new structure and redefined twist provided a more desirable flatter power curve on expense of high stress induction due to a larger area entering in to stall.

\subsubsection{Hinge_Twist_75_Combo_Sweptback}

Hinge_ Twist_ 75_Combo_Sweptback is a blade that is combination of two material hinges combined with the geometry of sweptback blade as reflected by its name. This blade was built upon the Combo blade configuration. The first hinge was active for $15 \%$ to $40 \%$ of the blade span length measured from the root. This hinge acted at an intensity of $20 \%$ for the hinge span and went to its $100 \%$ for the rest of the blade span. The changes of the hinge were reflected on the spar cap regions. The figure below 6.16 shows us the power outputs pf sweptback blade, original blade and Hinge_ Twist_75_Combo_Sweptback blade. The Hinge_ Twist_ 75_CCombo_Sweptback blade blade starts at a considerably lower power of 4.04 MW which is the lowest among the lot with original blade showing 5.16 MW and a power output of 4.745 MW of the Sweptback blade. As we move along the course of the power curve it is seen that the Hinge_ Twist_ 75_Combo_ Sweptback blade traces a more flat power curve than the default sweptback blade. At the mark of $15 \mathrm{~m} / \mathrm{s}$ where the sweptback blade shows a sudden change in slope reflecting stall and thereby stress inductions the Hinge_ Twist_75_COmbo_Sweptback blade blade follows the same course with negligible change in the gradient. The flow is still in continuous flow regime with no sudden drop of power due to loss of lift. Going ahead at higher wind speeds of $20 \mathrm{~m} / \mathrm{s}$ and $25 \mathrm{~m} / \mathrm{s}$ both blades show the same trends that they have been following from the $15 \mathrm{~m} / \mathrm{s}$ mark. The material adaptiveness us in action for all wind speeds. Comparing the power curves of Hinge_ Twist_ 75_Combo_Sweptback blade with Combo blade clearly reflects how, material adaptiveness has made the blades to behave in a similar fashion. In conclusion we can state that Hinge_ Twist_ 75_COmbo_Sweptback brings out, from material adaptiveness to avoid sudden change in flow regimes and stresses along with the geometrical adaptiveness of the sweptback blades. 


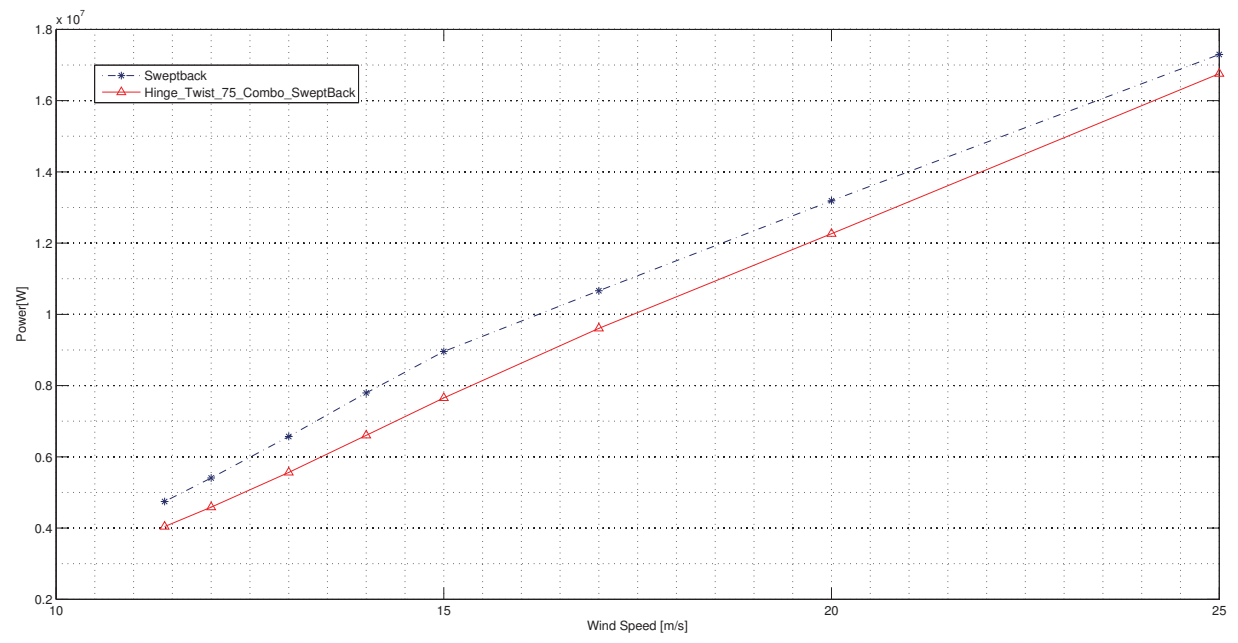

Figure 6.16. Power output of Sweptback and Hinge_ Twist_75_Combo_Sweptback.

\section{âĂ $\check{C}$}

\subsubsection{Hinge_Twist_75_Combo_Stall}

Hinge_Twist_75_Combo_Stall as the name suggests is made up of 2 material hinge as in the previous blade and the stall architecture of a stall blade. The two material hinges are exactly the same as that of the previous blade and help imposing the material adaptiveness on the stall blade. On comparing the power plots 6.176 .16 for Hinge_ Twist_ 75_Combo_ Sweptback and Hinge_ Twist_ 75_Combo_Stall we can see how flat the power output of the Hinge_ Twist_ 75_Combo_ Stall in comparison to Hinge_ Twist_ 75_ Combo_ Sweptback. It is clearly seen from the power plot that the combo stall blade remains flat until it reaches the mark of $20 \mathrm{~m} / \mathrm{s}$ when the wind speed is increased beyond $20 \mathrm{~m} / \mathrm{s}$ the Hinge_Twist_75_Combo_Stall blade is seen to activate its stall mode creating a more flat curve and thereby saving the generator to produce a constant output. This characteristic of the stall blade of generating a flat power curve when clubbed with the material adaptiveness of the hinged combinations gives us a Multi Concept Smart Blade. 


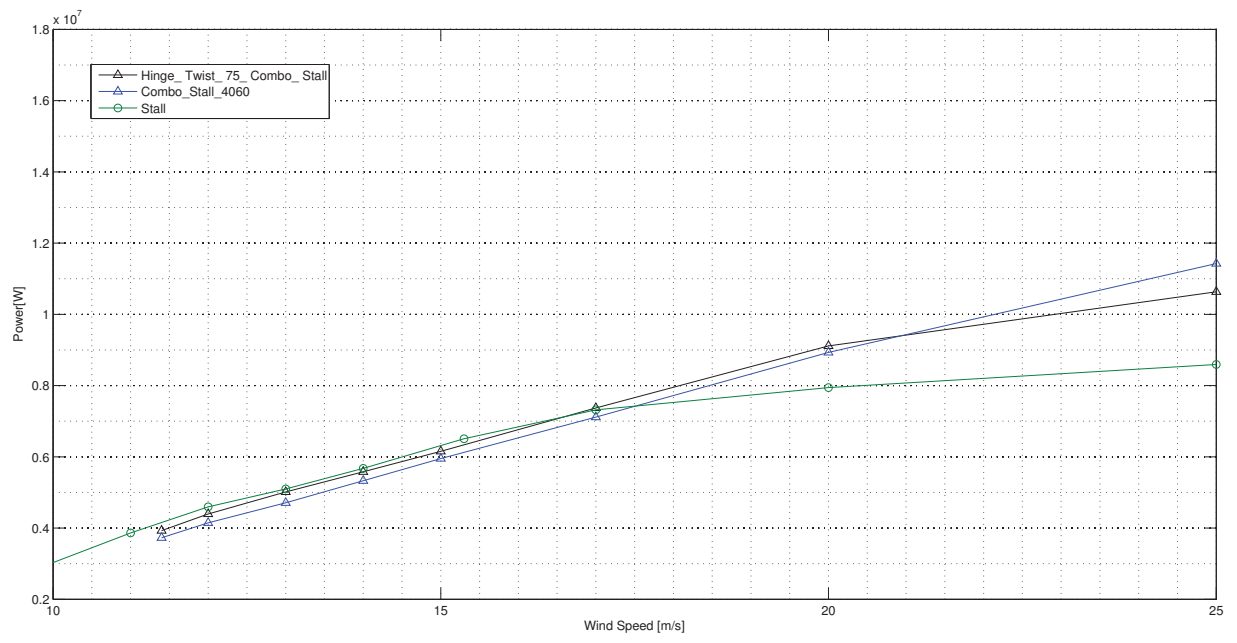

Figure 6.17. Power output of Stall, ComboStall and Hinge_ Twist_75_Combo_Stall. 


\section{Conclusions}

Conclusions The major aim of this dissertation was to generate benchmark materially adaptive wind turbine blades using the advanced computational tools developed by by the MTU's reserach team lead by Dr. Ponta. These tools were used for the aero elastic analysis of wind turbine blades and their subsequent experimentations. Along with the orthodox approach the inclusion of a fluid structure interaction algorithm to simulate the complex composite structure of these wind turbine blades made the model more advanced than its present counterparts like FAST-Aerodyne suite. The data obtained by the numerical experimentation of NREL-5MW Reference Wind Turbine was used as a datum to validate the codes for their authenticity. Once it was established that the results given out by our computational tools provided accurate data further experimentations on the grounds of stall, structural adaptiveness (bend-twist coupling), geometrical adaptiveness (swept-back), and their combinations were carried out.

The main aim to generating a Multi Concept Smart Blade (MCSB) was to obtain a blade that gave out the best of both worlds and thus provided us with a decently flatter power curve with less stress inductions on the blade thus increasing the blade durability. A common ODE frame work as discussed in Chapter2 was created for independent working of the solution. The whole process of obtaining the solution was classified into modules each having a specific function. The output of a certain module would in turn act as an input for the subsequent one hereby creating an efficient production chain. After establishing the path for solution we then explored into the 1-D flow model. This flow model provides us with the aerodynamic loads acting upon the blade span. Various coordinate systems and the effects of changing the coordinate system were also looked upon. All the flow parameters were established along with definition of the flow model interface. Instead of the classic BEM theory the structural model used involved the use of Generalized Timoshenko Beam theory GTBM developed by Dr. Otero which was explained in further details in Chapter 4. The dimensional reduction of the whole 3-D beam to a stiffness matrix of an equivalent 1-D beam was also accomplished. Once the solution is attained all the parameters of the 3-D blade structure are recovered from the 1-D beam.

We then move into the EXPERIMENTATION. We start off with a brief introduction ho 
w the blade is structured and its properties that are to be monitored. The whole blade was divided in to 20 slices. The properties of teach station were defined on the basis of the RWT. Appropriate airfoils were established at proper station locations to maintain coherence with the wind turbine tested by NREL. The blade as a whole as it would appear inside the codes was also looked upon. Transition airfoils, as in the ones that help in the transition of the airfoils form the completely cylindrical cross section in near Hub region at the point of attachment. A new 20 slice blade was obtained which was then converted into a 33 slice one to obtain more details both in terms of geometry as well as the properties contributing in gauging the exact behavior of the made blade. This also helps in achieving real like solutions. The problem definition was then set to be LAMBDA equals 7 thus making the tangential velocity at the tip to be $80 \mathrm{~m} / \mathrm{s}$. The validation tests for the steady state were carried out at the defined set of wind velocities. The velocities were distributed in such a way that the array would encompass most of the real time scenarios that a HAWT might experience in its working period.

After comparing the results of the original blade with the numerical data we then moved forward in inducing various modifications on different fronts like geometry, structure etc. The first of all the modifications was the superblade which was created as the first steps towards attaining material adaptiveness. This blade was made a tad bit softer to generate optimum working conditions for the blades. With the blade deflection well under the permissible limits this blade showed promising results as far as the material adpativness was considered, giving more or less a linear power curve thus overcoming the stalling of the wind turbine at higher velocities. Once the material adaptiveness was tried and tested we then moved on into targeting specific location on the blade. This is where we invented the material hinge. A material hinge is a section along the span of the blade where the material adaptivness is more active. Three blades with a single material hinge acting on different internal structural regions were created to start off with. EGGshell was the blade which caught our attention than the other two viz U-form and the Combo. This blade had a material hinge active on parts 15 to 40 percent region on the blade span. This blade however expressed a similar trend to its mother blade with a few minor changes like more linear power curve at higher wind velocities.

We then entered into dual material hinge configurations HingeTwist_75_Combo was the first of its kind to show promising results. HingeTwist_75_Combo had the first hinge which was active at the same location as it was on the Eggshell. The second hinge even though overlapping the first hinge was active only on the manufacturing angles of the fibers in the spar cap zone. The splitting of the blade into two regions along its chord made this hinge possible. This independent fiber orientation provided more control over blade flapwise stiffness. HingeTwist_75_Combo led the foundations of more vivid experiments.

We then wandered into the geometrical adaptations, tests were carried out with sweptback blades. The main challenged faced in designing of these blades was their interference 
with the tower. The blades were designed with this constraint. These swept back blades did stick to the connected flow regime even for higher velocities. It was this behavior of the sweptback blades that helped in consideration of further experimentation on these type of blades. Experimentation on stall blades was then started after the sweptback ones. The stall blades have a great tendency to curb power and provide a flatter power curve on expense of stress inductions. The preliminary experimentation with fixed speed stall blades by DELFT University revealed the same. The stall optimized blade had a changed twist of the blade which causes a drastic change in the geometry. Comparing the experimentation results of the stall blades with that of the feathering blades it became evident that when the feathering blades show promising results in the low wind velocity domain the stall blades excel in the high wind velocity domain.

After carefully reviewing more than 20 combinations in blade configuration we concluded that combining the features of the blades from different domain would yield desirable results. We then combined the Hinge_Twist_75_Combo blade with stall as well as swept back blades and thus Hinge_Twist_75_Combo_Stall and Hinge_Twist_75_Combo_Sweptback were born. The dual hinge configuration when combined with the geometrical modifications of the swept back blades as well as a completely different structure of the stall blades with its redefined twist thus acted together to develop more efficient blades. It was concluded that Hinge_Twist_75_Combo_Sweptback bought out the best of material adaptiveness to avoid sudden change of flow regime by feathering into the flow which when combined with the geometrical adaptiveness of the sweptback blades generated results more apt for the concept of these smart blades. Hinge_Twist_75_Combo_Stall was designed on the same design principles as that of the earlier blade and combined the dual hinge configuration with the redefined twsit of the stall blades. As seen earlier the capability of the stall blades to generate flat power curves at higher wind velocities when cumulated with the feathering capabilities of the material adaptiveness generate a power curve as desired off. These multi concept smart blades are just the first step in the region of material adaptiveness. Inclusion of other flow control devices would add further more active control devices creating true to its kind optimum blade. The future holds in store more analysis of these blades by considering the gravitational effects as well as aerodynamic load effects on these blades under real time working conditions. 


\section{REFERENCES}

(1) W. W. E. Association, et al., World wind energy half-year report 20014, WWEA: Bonn (2014).

(2) WWEA, World Wind Energy Report 2009, Report, World Wind Energy Association, 2010. www. wwindea. org.

(3) NREL, Wind Power Today, Report DOE/GO-102005-2115, U.S. Department of Energy, 2005.

(4) Enercon E-126 website [internet], [cited February 2012]. Available at http:// www . enercon. de/en-en/66.htm (2012).

(5) L. Chen, F. Ponta, L. Lago, Perspectives on innovative concepts in wind-power generation, Energy for Sustainable Development 15 (2011) 398-410.

(6) A. D. Otero, F. L. Ponta, Structural analysis of wind-turbine blades by a generalized timoshenko beam model, Journal of Solar Energy Engineering 132 (2010) 011015.

(7) L. I. Lago, Structural response and dynamics of fluid-structure-control interaction in wind turbine blades (2012).

(8) L. I. Lago, F. L. Ponta, A. D. Otero, Analysis of alternative adaptive geometrical configurations for the nrel-5 mw wind turbine blade, Renewable Energy 59 (2013) $13-22$.

(9) A. Otero, Análisis no-lineal del comportamiento estructural de sistemas avanzados de conversión eoloeléctrica, Ph.D. thesis, Facultad de Ingeniería, Universidad de Buenos Aires, National Lybrary, Buenos Aires, Argentina, 2008. In spanish.

(10) D. W. Hodges, Nonlinear Composite Beam Theory, AIAA, Reston, Virginia, 2006.

(11) W. Yu, D. H. Hodges, Generalized Timoshenko theory of the variational asymptotic beam sectional analysis, J. American Helicopter Society 50 (2005) 46-55. 
(12) J. Jonkman, S. Butterfield, W. Musial, G. Scott, Definition of a 5-MW reference wind turbine for offshore system development, National Renewable Energy Laboratory, NREL/TP-500-38060 (2009).

(13) L. F. Shampine, Numerical solution of ordinary differential equations, Chapman \& Hall, New York, USA, 1994.

(14) L. F. Shampine, M. K. Gordon, Computer Solution of Ordinary Differential Equations: the Initial Value Problem, W. H. Freeman, San Francisco, 1975.

(15) I. G. L.F. Shampine, S. Thompson, Solving ODEs with MATLAB, Cambridge University Press, 2003.

(16) G. F. C. A. M. Valli, A. L. Coutinho, Control strategies for timestep selection in finite element simulation of incompressible flows and coupled reaction-convectiondiffusion processes, International Journal for numerical methods in fluids 47 (2005) 201-231.

(17) L. Shampine, Implementation of implicit formulas for the solution of odes, SIAM Journal on Scientific and Statistical Computing 1 (1980) 103.

(18) L. Shampine, M. Reichelt, The matlab ode suite, SIAM journal on scientific computing 18 (1997) 1-22.

(19) D. A. Griffin, Blade System Design Studies Volume I: Composite Technologies for Large Wind Turbine Blades, Report SAND2002-1879, Sandia National Laboratories, 2002.

(20) V. L. Berdichevsky, Variational-asymptotic method of constructing a theory of shells, J. App. Math. and Mech. 43 (1979) 664-687.

(21) W. Yu, D. H. Hodges, V. Volovoi, C. E. S. Cesnik, On Timoshenko-like modeling of initially curved and twisted composite beams, Int. J. Sol. and Struct. 39 (2002) $5101-5121$.

(22) K. J. Bathe, Finite element procedures, Prentice Hall, Englewood Cliffs, New Jersey, USA, 1996.

(23) F. L. Ponta, The KLE method: a velocity-vorticity formulation for the Navier-Stokes equations, J. Applied Mechanics 73 (2006) 1031-1038.

(24) D. H. Hodges, Geometrically exact, intrinsic theory for dynamics of curved and twisted anisotropic beams, AIAA Journal 41 (2003) 1131-1137. 
(25) M. J. Patil, M. Althoff, Energy-consistent, Galerkin approach for the nonlinear dynamics of beams using mixed, intrinsic equations, in: AIAA/ASME/ASCE/AHE/ASC Structures, Structural Dynamics and Material Conference, Reston, Virginia, USA, AIAA, 2006, pp. 1-9.

(26) G. E. Karniadakis, E. T. Bullister, A. T. Patera, A spectral element method for solution of two- and three-dimensional time-dependent incompressible navier-stokes equations, in: Finite Element Methods for Nonlinear Problems, Springer-Verlag, New York/Berlin, 1985, p. 803.

(27) A. T. Patera, A spectral element method for fluid dynamics: laminar flow in a channel expansion, J. Comput. Phys. 54 (1984) 468-488.

(28) L. E. Malvern, Introduction to the mechanics of a continuous medium, Prentice Hall, 1969.

(29) L. P. Kollár, G. S. Springer, Mechanics of Composites Structures, Cambridge University Press, New York, USA, 2003.

(30) V. V. Vasiliev, E. V. Morozov, Mechanics and Analysis of Composite Materials, Elsevier, Oxford, UK, 2001.

(31) G. Wempner, D. Talaslidis, Mechanics of solids and shells: theories and approximations, CRC Press LLC, Boca Raton, USA, 2003.

(32) P. Moriarty, A. Hansen, AeroDyn theory manual, National Renewable Energy Laboratory (2005).

(33) D. Laino, A. Hansen, User's Guide to the Wind Turbine Aerodynamics Computer Software AeroDyn, Report for National Renewable Energy Laboratory under subcontract No. TCX-9-29209-01 (2002).

(34) J. Jonkman, Minutes from the aerodyn overhaul kick-off meeting [internet], [cited June 2009]. Available at http://wind.nrel.gov/public/ jjonkman/AeroDynOverhaulKickOffMeeting (2008).

(35) H. Glauert, Airplane propellers, Aerodynamic theory 4 (1935) 169-360.

(36) J. Manwell, J. McCowan, A. Rogers, Wind Energy Explained: theory, design and application, Chichester ; New York : Wiley, 2002.

(37) T. Burton, Wind Energy Handbook, Wiley, 2001.

(38) D. Pitt, D. Peters, Theoretical prediction of dynamic-inflow derivatives, Vertica 5 (1981) 21-34. 
(39) J. Leishman, Principles of helicopter aerodynamics, Cambridge University Press, Cambridge, UK, 2006.

(40) C. Bak, H. Aagaard Madsen, J. Johansen, Influence from blade-tower interaction on fatigue loads and dynamics (poster), in: Wind energy for the new millennium. Proceedings. 2001 European wind energy conference and exhibition (EWEC’01), pp. 2-6.

(41) S. Powles, The effects of tower shadow on the dynamics of a horizontal-axis witn turbine, Wind Engineering 7 (1983) 26-42.

(42) K. Rao, Classical mechanics, Universities Press, 2003.

(43) H. Goldstein, Classical Mechanics, 2nd ed., Addison-Wesley, 1980.

(44) L. S. B. Siciliano, L. Villani, Robotics: modelling, planning and control, Springer Verlag, 2009.

(45) IEC, Wind Turbine Generator Systems - Part 13: Measurement of mechanical loads, Report IEC/TS 61400-13, International Electrotechnical Commission (IEC), 2001.

(46) Frenet-serret formulas [internet], [cited December 2011]. Available at http: / / en . wikipedia.org/wiki/Frenet-Serret_formulas (2011).

(47) F. Yamaguchi, Curves and surfaces in computer aided geometric design, SpringerVerlag Berlin, 1988.

(48) R. L. Burden, J. D. Faires, Numerical analysis, Brooks Cole, 1998.

(49) J. H. Mathews, K. D. Fink, Numerical methods using Matlab, Prentice Hall, 1999.

(50) H. Glauert, A general theory of the autogyro., British ARC 1111 (1926).

(51) M. Buhl, A new empirical relationship between thrust coefficient and induction factor for the turbulent windmill state, National Renewable Energy Laboratory, 2005.

(52) B. Lock, Townend, An extension of the vortex theory of airscrews with applications to airscrews of small pitch, including experimental results, Aeronautical Research Committee, R\&M 1014 (1925) 1 to 49.

(53) C. Hansen, Nwtc design codes (airfoilprep) [internet], [cited December 2011]. Available at http://wind.nrel.gov/designcodes/preprocessors/ airfoilprep/Last modified 09-March-2010 (2011). 
(54) Z. Du, M. Selig, A 3-D stall-delay model for horizontal axis wind turbine performance prediction, in: AIAA, Aerospace Sciences Meeting and Exhibit, 36 th, and 1998 ASME Wind Energy Symposium, Reno, NV; UNITED STATES, Reston, VA/New York: American Institute of Aeronautics and Astronautics, Inc./ASME International, pp. 9-19.

(55) A. Eggers, Modeling of yawing and furling behavior of small wind turbines, in: 2000 ASME Wind Energy Symposium, 19 th, AIAA, Aerospace Sciences Meeting and Exhibit, 38 th, Reno, NV, pp. 1-11.

(56) L. Viterna, D. Janetzke, Theoretical and experimental power from large horizontalaxis wind turbines, NASA STI/Recon Technical Report N 82 (1982).

(57) J. Jonkman, M. Buhl Jr, Fast users guide, Rep. No. NREL/EL-500-38230, NREL, Golden, Colorado, USA (2005).

(58) H. Kooijman, C. Lindenburg, D. Winkelaar, E. van der Hooft, DOWEC 6 MW predesign, Technical Report, ECN-CX-01-135, Energy Research Center of the Netherlands, Petten, 2003.

(59) C. Lindenburg, Aeroelastic modelling of the lmh64-5 blade, ECN, Petten, December (2002).

(60) Iea wind, subtask 2: Research for deeper waters [internet], [cited December 2011]. Available at http://www. ieawind.org/AnnexXXIII/Subtask2. html (2011).

(61) Upwind website [internet], [cited December 2011]. Available at http://www . upwind.eu/default.aspx (2011).

(62) J. Jonkman, S. Butterfield, P. Passon, T. Larsen, T. Camp, J. Nichols, J. Azcona, A. Martinez, Offshore code comparison collaboration within iea wind annex xxiii: Phase ii results regarding monopile foundation modeling, in: 2007 European Offshore Wind Conference \& Exhibition, 4-6 December 2007, Berlin, Germany.

(63) P. Passon, M. Kühn, S. Butterfield, J. Jonkman, T. Camp, T. Larsen, OC3-Benchmark exercise of aero-elastic offshore wind turbine codes, in: Journal of Physics: Conference Series, volume 75, IOP Publishing, p. 012071.

(64) W. A. Timmer, R. P. J. O. M. van Rooij, Summary of the Delft University wind turbine dedicated airfoils, in: 41st Aerospace Sciences Meeting and Exhibit, AIAA, Reno, Nevada.

(65) W. A. Timmer, Personal communication, 2007.

(66) I. TPI Composites, Parametric study for large wind turbine blades, Report SAND2002-2519, Sandia National Laboratories, 2002. 


\section{APPENDICES}




\section{APPENDIX A. Copyright AgreEMENTS}

\section{A.1 Copyright statement for Chapter 1}

Pictures from chapter 1 are reproduced from Wikimedia Commons, a freely licensed media file repository. Files are licensed under the Creative Commons Attribution-Share Alike 2.0 and/or 3.0 Unported license. According to these license, permission is granted to:

1. Share, copy, distribute and transmit the work.

2. Remix, adapt the work.

Under the following conditions:

1. Attribution You must attribute the work in the manner specified by the author or licensor (but not in any way that suggests that they endorse you or your use of the work).

2. share alike If you alter, transform, or build upon this work, you may distribute the resulting work only under the same or similar license to this one.

ATTRIBUTIONS:

- Picture from figure 1.3a was taken by Hans Hillewaert. Source and permissions availables at: http://en.wikipedia.org/wiki/File:Windmills_D1-D4_ 28Thornton_Bank29.jpg, under CC-BY-SA-3.0.

- Picture from figure 1.3b was taken by Kuebi = Armin KÃijbelbeck. Source and permissions availables at: http://commons.wikimedia.org/wiki/File: Schneebergerhof_05.jpg, 
- Picture from figure 1.4 was taken by Korona B. Source and permissions availables at: http://en.wikipedia.org/wiki/File:Farma_wiatrak 03 ○B3w_Bukowsko_.JPG, under CC-BY-SA-3.0.

- Picture from figure 1.5 was taken by Paul Anderson. Source and permissions availables at: http://en.wikipedia.org/wiki/File:Turbine_ Blade_Convoy_Passing_through_Edenfield.jpg, under CC-BY-2.0. 